\title{
A SURVEY OF INTEGRAL REPRESENTATION THEORY ${ }^{1}$
}

\author{
BY IRVING REINER
}

\section{TABLE OF CONTENTS}

1. Introduction. Notation and definitions..................... 159

2. General remarks. Jordan-Zassenhaus Theorem.................. 162

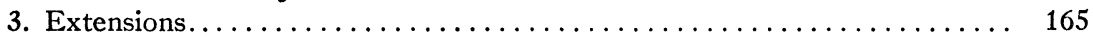

4. Higman ideal................................... 168

5. Representations over local domains...................... 171

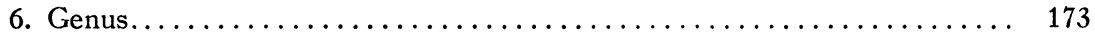

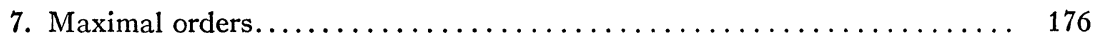

8. Further results on genera........................... 182

9. Projective modules and relative projective modules.............. 186

10. Grothendieck groups and Whitehead groups................... 189

11. Commutative orders and related results.................. 196

12. Divisibility of modules.............................. 200

13. Hereditary orders and related results.................... 201

14. Finiteness of the number of indecomposable representations.......... 205

15. Representations of specific groups and orders............... 207

16. Representation rings................................ 208

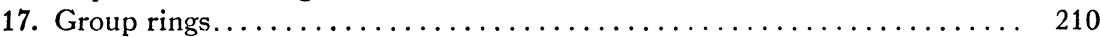

18. Algebraic number theory............................. 212

19. Krull-Schmidt and Cancellation Theorems................... 213

1. Introduction. Notation and definitions. First of all I wish to acknowledge with thanks the many helpful conversations I have had with Professors Olga Taussky, Peter Roquette and Hans Zassenhaus, when I first began studying the subject of integral representations.

Historically, the subject received its main impetus from two branches of algebra. One branch is algebraic number theory, especially that part concerned with ideal theory; and the other is matrix theory, mainly that portion dealing with matrix representations of associative algebras. Methods of homological algebra have played an increasingly important role in the subject in recent years.

An expanded version of an address delivered before the Chicago meeting of the Society by invitation of the Committee to Select Hour Speakers for Western Sectional Meetings, April 20,1968, under the title Recent progress in the theory of integral representations; received by the editors September 17, 1969. 1620.

AMS Subject Classifications. Primary 1075, 1548, 2080, 1640; Secondary 1069,

Key Words and Phrases. Integral representations, orders, representation lattices, Grothendieck groups, noncommutative arithmetic, indecomposable representations, group representations, genus.

${ }^{1}$ This work was partially supported by a research contract with the National Science Foundation. 
We wish to study representations of certain algebraic systems, called orders, by means of matrices with entries in an integral domain $R$. It is often more convenient to consider, instead of matrices, the underlying spaces on which these matrices act. Such spaces are special instances of $R$-lattices, by which we mean finitely generated torsionfree $R$-modules. Only those $R$-lattices having a free $R$-basis give rise to matrices. Of course, when $R$ is a principal ideal domain, every $R$-lattice has a free $R$-basis.

Let $\Lambda$ be a ring with unity element 1 , containing $R$ in its center (identify $R$ with $R \cdot 1$ ). This makes $\Lambda$ into an $R$-module. Call $\Lambda$ an $R$-order if $\Lambda$ is finitely generated and torsionfree as $R$-module. Let $F$ be the quotient field of $R$, and set $A=F \otimes_{R} \Lambda$, so that $A$ is a finite dimensional algebra over $F$. We always identify $\Lambda$ with $1 \otimes \Lambda$, so $\Lambda$ is embedded in $A$, and we may write $A=F \cdot \Lambda$ (the set of $F$-linear combinations of the elements of $\Lambda$ ). Likewise, every $R$-lattice $M$ is embedded in the vector space over $F$ given by $F \otimes_{R} M=F M$. The dimension $(F M: F)$ is called the $R$-rank of $M$.

Here are some examples of orders:

1. The ring of all algebraic integers in an algebraic number field $F$ is a $Z$-order in $F$, and will be denoted hereafter by alg. int. $\{F\}$. We shall use this notation only when $F$ is an algebraic number field.

2. If $G$ is a finite group, the integral group ring $R G$ is an $R$-order in the group algebra $F G$.

3. The ring $(R)_{n}$ of all $n \times n$ matrices with entries in $R$ is an $R$-order in the matrix algebra $(F)_{n}$.

4. For $\alpha$ an algebraic integer, the ring $Z[\alpha]$ is a $Z$-order in the field $Q(\alpha)$, where $Q$ is the rational field.

Let $\Lambda$ be an $R$-order; a $\Lambda$-lattice is an $R$-lattice which is also a $\Lambda$-module. The fundamental problem in the theory of integral representations is as follows:

Given an $R$-order $\Lambda$, determine all $\Lambda$-lattices. Once we have obtained some answers to this question, we may then proceed to the next problem:

Apply the theory of integral representations to investigate properties of various orders.

The purpose of this survey is to describe the present state of knowledge about these problems, especially the first one. References which give a general introduction to the theory of orders are $\mathrm{CR}^{2}$ Chapter 11, Roggenkamp-Dyson [208b], Faddeev [80].

\footnotetext{
${ }^{2} \mathrm{CR}$ denotes the reference Curtis-Reiner [46] throughout.
} 
It is clearly an impossible task to give a complete description of the contents of the 250 or so bibliographical references for this article. I have of course been guided by my own interests in making a selection from the wealth of material available, and I regret that many references are mentioned only briefly in passing. Certain important topics on the periphery of the subject have been omitted entirely, and would require their own survey articles; in particular, there is no discussion of cohomology of groups, nor of Galois theory of rings.

In addition to the earlier notation, we also fix the following once and for all:

char $F$ : characteristic of the field $F$

$P=$ maximal ideal of the integral domain $R$

$R_{P}=$ localization of $R$ at $P=\{\alpha / \beta: \alpha \in R, \beta \in R-P\}$

$R_{P}^{*}=P$-adic completion of $R ; F_{P}^{*}=$ quotient field of $R_{P}^{*}$

$M^{(k)}=$ direct sum of $k$ copies of $M$

$(D)_{n}=$ ring of all $n \times n$ matrices with entries in $D$

$G=$ finite group of order $g$

+ : external direct sum

$\sum: \oplus$ : internal direct sum

$a \mid b: a$ divides $b$

$a \nmid b: a$ does not divide $b$

$\operatorname{rad} \Lambda=$ Jacobson radical of the ring $\Lambda$

A free left $\Lambda$-module is a direct sum of copies of the left $\Lambda$-module $\Lambda$. A projective left $\Lambda$-module is a direct summand of a free module. The ring $\Lambda$ is left hereditary if every left ideal of $\Lambda$ is a projective $\Lambda$-module. (Equivalently, $\Lambda$ is left hereditary if every submodule of a free $\Lambda$-module is isomorphic to an external direct sum of left ideals of $\Lambda$, each of which is projective. M. Auslander has shown that if $\Lambda$ is both left and right noetherian, then $\Lambda$ is left hereditary if and only if $\Lambda$ is right hereditary. See Rotman [218], Cartan-Eilenberg [40].)

A Dedekind domain is an integral domain in which every nonzero ideal is uniquely expressible as a product of prime ideals. (Equivalently, a Dedekind domain is a hereditary integral domain. See Cartan-Eilenberg [40].) In particular alg. int. $\{F\}$ is always a Dedekind domain.

(1.1). Definition. Let $R$ be a Dedekind domain. An $R$-ideal in $F$ is a nonzero $R$-lattice in $F$. Products of $R$-ideals are again $R$-ideals; relative to this multiplication, the $R$-ideals in $F$ form a multiplicative group denoted by $I\{R\}$.

The following theorem lists some standard properties of lattices over Dedekind domains. 
(1.2). Theorem. Let $R$ be a Dedekind domain.

(i) Every $R$-lattice is a projective $R$-module.

(ii) Let $N \subset M$ be R-lattices. Then $N$ is an R-direct summand of $M$ if and only if $M / N$ is $R$-torsionfree.

(iii) Each $R$-ideal $J$ in $F$ can be generated (as $R$-module) by two elements. Further, each $J$ is invertible, that is, there is an $R$-ideal $J^{\prime}$ such that $J \cdot J^{\prime}=R$.

(iv) Given any $R$-lattice $M$, there exist elements $m_{1}, \cdots, m_{k} \in M$ and ideals $J_{1}, \cdots, J_{k} \in I\{R\}$ such that

$$
M=J_{1} m_{1} \oplus \cdots \oplus J_{k} m_{k} .
$$

(v) Given R-lattices $N \subset M$, there exist elements $m_{1}, \cdots, m_{k} \in M$, $R$-ideals $J_{1}, \cdots, J_{k} \in I\{R\}$, and integral ideals $E_{1}, \cdots, E_{k}$ in $R$ (some of which may be zero), such that

$$
\begin{aligned}
M & =J_{1} m_{1} \oplus \cdots \oplus J_{k} m_{k}, \\
N & =E_{1} J_{1} m_{1} \oplus \cdots \oplus E_{k} J_{k} m_{k}, \\
E_{1} & \supset E_{2} \supset \cdots \supset E_{k} .
\end{aligned}
$$

References. Cartan-Eilenberg [40, Chapter 7], Chevalley [41], CR Chapter 3, Kaplansky [138], [139], Levy [152], Steinitz [228], Zariski-Samuel [262, Chapter 5].

Let $J, J^{\prime} \in I\{R\}$; they are in the same ideal class if $J^{\prime}=J x$ for some $x \in F$. The number of ideal classes is called the (ideal) class number of $R$ (or of $F$ ), and is finite when $F$ is an algebraic number field. (CR Chapter 3).

Now let $N \subset M$ be $R$-lattices for which $M / N$ is a torsion $R$-module. Then each of the ideals $\left\{E_{i}\right\}$ occurring in part $(\mathrm{v})$ of Theorem 1.2 is nonzero, and we define the order ideal of $M / N$ to be the product $E_{1} \cdots E_{k}$. Equivalently, this order ideal is the product of the $R$-annihilators of the composition factors of the $R$-module $M / N$. Denote by $\operatorname{ord}(M / N)$ the order ideal of $M / N$. References for this concept: Fröhlich [85], CR §80.

2. General remarks. Jordan-Zassenhaus Theorem. To begin with, let $R$ be an arbitrary noetherian domain, and $\Lambda$ an $R$-order in the $F$-algebra $A$. Given a $\Lambda$-lattice $M$, form the $A$-module $F \otimes_{R} M$. Identifying $M$ with $1 \otimes M$, we write $F \cdot M$ in place of $F \otimes M$. Such identifications will be made hereafter without specific comment.

Let $N \subset M$ be $\Lambda$-lattices, and call $N R$-pure in $M$ if $M / N$ is $R$ torsionfree. An easy result is 
(2.1). Theorem. Given any $\Lambda$-lattice $M$, there is a one-to-one correspondence $W \leftrightarrow N$ between $A$-submodules $W$ of $F M$ and $R$-pure $\Lambda$ sublattices $N$ of $M$. The correspondence is given by

$$
N=M \cap W, \quad W=F N .
$$

Further, each finitely generated $A$-module $V$ is of the form $F M$ for some $\Lambda$-lattice $M$ in $V$.

References. Zassenhaus [263]; CR \$73.

The latter part of the above theorem has important consequences for the theory of group representations. Let $G$ be a finite group; an $F$-representation of $G$ of degree $n$ is a homomorphism of $G$ into $(F)_{n}$, carrying the identity of $G$ onto the identity matrix. Two $F$-representations $T: G \rightarrow(F)_{n}, U: G \rightarrow(F)_{n}$ are $F$-equivalent if there exists an invertible $C \in(F)_{n}$ such that

$$
C^{-1} \cdot T(x) \cdot C=U(x) \text { for all } x \in G .
$$

In other words, $T$ and $U$ are equivalent if they are afforded by the same $F G$-module by using different $F$-bases of that module.

Taking $\Lambda=R G$ in the latter part of (2.1), we obtain

(2.2). THEOREM. If $R$ is a principal ideal domain, every F-representation of the finite group $G$ is $F$-equivalent to an $R$-representation of $G$.

Even when $R$ is not a principal ideal domain, the above result is useful. In particular, suppose $R=$ alg. int. $\{F\}$, and let $R^{\prime}=\operatorname{alg}$. int. $\left\{F^{\prime}\right\}$ for some $F^{\prime} \supset F$. It is known that $F^{\prime}$ may be chosen so that for each $J \in I\{R\}$, the $R^{\prime}$-ideal $J R^{\prime}$ is principal. It is also known that every complex representation of a finite group $G$ is equivalent (over the complex field) to an $F$-representation of $G$, for some algebraic number field $F$. This yields

(2.3). Theorem. Every complex representation of $G$ is equivalent (over the complex field) to a representation by matrices whose entries are algebraic integers.

Reference. CR (75.4).

In this direction, we quote a result due to Schur.

(2.4). TheOrem. Let $R=$ alg. int. $\{F\}$, and let $h$ be the number of ideal classes in $R$. If $(h, n)=1$, then every F-representation of $G$ of degree $n$ is $F$-equivalent to an $R$-representation of $G$.

Reference. CR (75.5). 
Returning now to the general case where $\Lambda$ is an $R$-order in $A$, we start with any $\Lambda$-lattice $M$, and write a composition series for the $A$-module $F M$, say

$$
F M=W_{0} \supset W_{1} \supset \cdots \supset W_{k}=0 .
$$

Then there is a chain of $\Lambda$-lattices

$$
M=M_{0} \supset M_{1} \supset \cdots \supset M_{k}=0, \quad M_{i}=M \cap W_{i} .
$$

For each $i, M_{i+1}$ is an $R$-pure $\Lambda$-sublattice of $M_{i}$ such that

$$
F \otimes_{R}\left(M_{i} / M_{i+1}\right) \cong W_{i} / W_{i+1} \text {. }
$$

We may call (2.5) an " $R$-composition series" for $M$, with " $R$-composition factors" $\left\{M_{i} / M_{i+1}: 0 \leqq i<k\right\}$. The analogue of the JordanHölder Theorem is not valid, however, since indeed the $R$-composition factors are (in general) not uniquely determined by $M$. (See Reiner [184]; CR, Example 2 at the end of \$73.)

As is well known, the number of ideal classes in alg. int. $\{F\}$ is finite (see CR, $\$ 20$, for example). A far-reaching generalization of this is the fundamental result

(2.6). Jordan-Zassenhaus Theorem. Let $R$ be a Dedekind domain whose quotient field $F$ is an algebraic number field, and let $\Lambda$ be an $R$-order in a semisimple F-algebra $A$. Let $W$ be any finitely generated $A$-module, and set

$$
\sigma(W)=\{M: M=\Lambda \text {-lattice, } F M \cong W\} .
$$

Then the number $s(W)$ of $\Lambda$-isomorphism classes in $\sigma(W)$ is finite.

References. Zassenhaus [263], CR §79. For corresponding results when $R=k[X]$, where $X$ is an indeterminate over the finite field $k$, see Roggenkamp [202], [203]. For the more general case where $R$ is the integral closure of $k[X]$ in a finite extension of $k(X)$, see HigmanMcLaughlin [122]. The case where $R$ is a $P$-adic ring is treated by Jenner [134].

The actual determination of $s(W)$ is a difficult problem. The most fruitful approach is to partition $\sigma(W)$ into certain collections of $\Lambda$-isomorphism classes, called genera. We place two $\Lambda$-lattices $M$ and $N$ in the same genus if and only if

(2.7). $F M \cong F N$ and $R_{P} M \cong R_{P} N$ for each maximal ideal $P$ of $R$.

It is sometimes possible to determine explicitly the number of genera in $\sigma(W)$, and then to find the number of $\Lambda$-isomorphism classes in each genus. We shall discuss this in more detail in $\$ \S 6,8$. 
We give some examples where $s(W)$ can be calculated. For instance, suppose

$$
\Lambda=Z[x] /\left(x^{n}-1\right)
$$

so $\Lambda \cong Z G, G$ cyclic of order $n$. Let $W=Q\left(\theta_{d}\right)$, the cyclotomic field generated by a primitive $d$ th root of unity $\theta_{d}$, where $d \mid n$. Then $W$ is an irreducible $A$-module, and each $M \in \sigma(W)$ is $\Lambda$-isomorphic to a $Z\left[\theta_{d}\right]$-ideal in the field $Q\left(\theta_{d}\right)$. In this case, $s(W)$ is precisely the number of ideal classes in the ring $Z\left[\theta_{d}\right]$. (See also Taussky-Todd [250], [251].)

In this direction, let us consider the case where $f(x)$ is a monic $n$th degree polynomial in $Z[x]$ with distinct zeros. Set

$$
A=Q[x] /(f(x)), \quad \Lambda=Z[x] /(f(x)),
$$

so $\Lambda$ is a $Z$-order in $A$. Any $X \in(Q)_{n}$ with minimal polynomial $f(x)$ gives rise to an algebra isomorphism $A \cong Q[X]$ via $x \rightarrow X$, and so this map is just a $Q$-representation of $A$ afforded by $A$ itself. Those matrices $M \in(Z)_{n}$ with minimal polynomial $f(x)$ are afforded by $\Lambda$-lattices in $A$. Isomorphism classes of lattices correspond to classes of integral matrices under unimodular equivalence, where the matrices $M, N$ are unimodularly equivalent if there exists a matrix $U \in(Z)_{n}$ such that $U^{-1} \in(Z)_{n}$, and $M=U N U^{-1}$. Further, two $\Lambda$-lattices $L, L^{\prime}$ are isomorphic if and only if $L^{\prime}=L \lambda$ for some unit $\lambda \in A$, so the number of isomorphism classes of lattices is the same as the number of left ideal classes in $\Lambda$.

These results, due to Latimer-MacDuffee [148], were simplified by Taussky [240] in case $f(x)$ is irreducible. In that case, let $\alpha$ be an algebraic integer for which $f(\alpha)=0$, so $A=Q(\alpha)$ is a field, and $\Lambda$ $=Z[\alpha]$ is a $Z$-order in $A$. Each ideal $m$ of $\Lambda$ gives rise to a matrix $M$ (with $f(M)=0$ ) by letting $\alpha$ act on a $Z$-basis for $\mathfrak{m}$. Assuming that $\Lambda=$ alg. int. $\{A\}$, Taussky [244] proved that the ideal class of $\mathfrak{m}^{-1}$ corresponds to the transpose $M^{\top}$. Further, let $X \in(Z)_{n}$ carry the $Z$-basis $\left\{1, \alpha, \cdots, \alpha^{n-1}\right\}$ of $\Lambda$ onto a $Z$-basis of the ideal $m$; such a matrix $X$ is an ideal matrix. Taussky studied such ideal matrices in detail in [246] and [247]; see also [248] for a description of matrices $U$ such that $M^{\top}=U M U^{-1}$.

Other references for this section: Bender [15], Dade [49].

3. Extensions. As general references for this section we cite Cartan-Eilenberg [40], CR, Rotman [218]. We assume throughout that $R$ is a noetherian domain, and that $\Lambda$ is any $R$-order. Given a pair of $\Lambda$-lattices $N, L$, we may form the finitely generated $R$-module 
$\operatorname{Ext}_{\Lambda}^{1}(N, L)$, whose elements are in one-to-one correspondence with classes of exact sequences of $\Lambda$-lattices $0 \rightarrow L \rightarrow M \rightarrow N \rightarrow 0$. Two such sequences are placed in the same class if there is a commutative diagram

$$
\begin{aligned}
0 \rightarrow L & \rightarrow M \rightarrow N \rightarrow 0 \\
1 \downarrow & \downarrow \quad 1 \downarrow \\
0 \rightarrow L & \rightarrow M^{\prime} \rightarrow N \rightarrow 0 .
\end{aligned}
$$

(Addition of extensions is gotten from the Baer sum, and the elements of $R$ act on Ext by acting on either one of the lattices.)

Hence if we are given a pair of $\Lambda$-lattices $L$ and $N$, and wish to determine all $\Lambda$-lattices $M$ containing $L$ such that $M / L \cong N$, we must first calculate $\operatorname{Ext}_{\Lambda}^{1}(N, L)$, and then decide when extensions $M$ from different classes are $\Lambda$-isomorphic. (See Reiner [183], for example.)

Let $R_{P}$ denote the localization of $R$ at a maximal ideal $P$ of $R$. For each $R$-module $T$, we may set $T_{P}=R_{P} \otimes_{R} T$. Then $\Lambda_{P}$ is an $R_{P}$-order, and each $\Lambda$-lattice $L$ determines a $\Lambda_{P}$-lattice $L_{P}$.

(3.1). Theorem. Let $L, N$ be $\Lambda$-lattices, and let $P$ range over the set of maximal ideals of $R$. Then:

(i) For each $P$,

$$
\left\{\operatorname{Ext}_{\Lambda}^{1}(N, L)\right\}_{P} \cong \operatorname{Ext}_{\Lambda_{P}}^{1}\left(N_{P}, L_{P}\right) .
$$

(ii) There is a monomorphism

$$
\operatorname{Ext}_{\Lambda}^{1}(N, L) \rightarrow \prod_{P} \operatorname{Ext}_{\Lambda_{P}}^{1}\left(N_{P}, L_{P}\right) .
$$

(iii) If $R$ is a Dedekind domain, and if there exists a nonzero ideal $J$ in $R$ such that $J \cdot \operatorname{Ext}_{\Lambda}^{1}(N, L)=0$, then

$$
\operatorname{Ext}_{\Lambda}^{1}(N, L) \cong \sum_{P \supset J} \cdot \operatorname{Ext}_{\Lambda_{P}}^{1}\left(N_{P}, L_{P}\right)
$$

(iv) If $R_{P}^{*}$ denotes the $P$-adic completion of $R_{P}$, then

$$
R_{P}^{*} \otimes_{R_{P}} \operatorname{Ext}_{\Lambda_{P}}^{1}\left(N_{P}, L_{P}\right) \cong \operatorname{Ext}_{\Lambda_{P}^{*}}^{1}\left(N_{P}^{*}, L_{P}^{*}\right) .
$$

References. CR (75.25), Auslander-Goldman [1], Cartan-Eilenberg [40], DeLeeuw [54], Nunke [74], Reiner [183]. We remark that all of the above isomorphisms are natural.

An exact sequence of $\Lambda$-lattices

$$
0 \rightarrow L \stackrel{f}{\rightarrow} M \stackrel{g}{\rightarrow} N \rightarrow 0
$$


is said to be split if $f(L)$ is a $\Lambda$-direct summand of $M$, or equivalently, if there exists a map $h \in \operatorname{Hom}_{\Lambda}(N, M)$ such that $g h=$ identity map on $N$. From the definition of Ext, it follows at once that the sequence (3.2) is split if and only if it represents the zero class in $\operatorname{Ext}_{\Lambda}^{1}(N, L)$.

For each maximal ideal $P$ of $R$, tensoring (3.2) with $R_{P}$ yields an exact sequence of $\Lambda_{P}$-lattices

$$
0 \rightarrow L_{P} \underset{f_{P}}{\rightarrow} M_{P} \underset{g_{P}}{\rightarrow} N_{P} \rightarrow 0 .
$$

Then (ii) of (3.1) may be rephrased as follows: the sequence (3.2) splits if and only if (3.3) splits for each $P$.

Suppose now that the exact sequence (3.2) of $\Lambda$-lattices is split as a sequence of $R$-modules. Then every element of $M$ can be represented uniquely as an ordered pair $(l, n), l \in L, n \in N$. The action of the elements of $\Lambda$ is given by a formula

$$
x(l, n)=\left(x l+F_{x} n, x n\right), \quad x \in \Lambda,
$$

where $F_{x} \in \operatorname{Hom}_{R}(N, L)$. We have thus obtained an $R$-homomorphism

$$
F: \Lambda \rightarrow \operatorname{Hom}_{R}(N, L),
$$

given by $x \rightarrow F_{x}$, and it is easily seen that $F$ satisfies the condition

$$
F_{x y}=x \cdot F_{y}+F_{x} \cdot y, \quad x, y \in \Lambda .
$$

Conversely, any $R$-homomorphism $F$ (as in (3.5)) for which (3.6) holds gives rise to an extension of $N$ by $L$ by means of formula (3.4). Zassenhaus [263] calls $F$ a binding system. For each $t \in \operatorname{Hom}_{R}(N, L)$, the map $x \rightarrow x t-t x, x \in \Lambda$, is an inner binding system. Assuming that $N$ is $R$-projective, we have

$$
\operatorname{Ext}_{\Lambda}^{1}(N, L) \cong \text { binding systems/inner binding systems. }
$$

This holds in particular whenever $R$ is a Dedekind domain, and $N$ is any $R$-lattice.

Assuming now that $R$ is a Dedekind domain, let $M$ be any left $\Lambda$-lattice, and set $M^{*}=\operatorname{Hom}_{R}(M, R)$. Then $M^{*}$ becomes a right $\Lambda$-lattice by means of the formula

$$
\left(m^{*} x\right) m=m^{*}(x m), \quad m^{*} \in M^{*}, x \in \Lambda, m \in M .
$$

There is a natural isomorphism $\left(M^{*}\right)^{*} \cong M$ as left $\Lambda$-lattices. Every exact sequence (3.2) of left $\Lambda$-lattices gives rise to an exact sequence $0 \rightarrow N^{*} \rightarrow M^{*} \rightarrow L^{*} \rightarrow 0$ of right $\Lambda$-lattices. Thus there is an $R$-isomorphism

$$
\operatorname{Ext}_{\Lambda}^{1}(N, L) \cong \operatorname{Ext}_{\Lambda}^{1}\left(L^{*}, N^{*}\right)
$$


Consider now the special case where $\Lambda=R G$, and let $M$ be any left $\Lambda$-lattice. The above-defined $M^{*}$ can then be made into a left $R G$ lattice $M^{\prime}$, called the contragredient of $M$, by defining

$$
\left(x m^{*}\right) m=m^{*}\left(x^{-1} m\right), \quad m^{*} \in M^{\prime}, x \in G, m \in M .
$$

(If $M$ affords a matrix representation $x \rightarrow M(x), x \in G$, then $M^{\prime}$ affords the representation $x \rightarrow\left(M\left(x^{-1}\right)\right)^{\top}$ where T denotes transpose.)

It is easily seen that $(R G)^{\prime} \cong R G$ as left $R G$-modules, and that there is an $R$-isomorphism

$$
\operatorname{Ext}_{R G}^{1}(N, L) \cong \operatorname{Ext}_{R G}^{1}\left(L^{\prime}, N^{\prime}\right)
$$

for any pair of left $R G$-lattices $N, L$.

Call a left $R G$-lattice $L$ weakly injective if every exact sequence $0 \rightarrow L \rightarrow M \rightarrow N \rightarrow 0$ is $R G$-split, where $M, N$ are arbitrary $R G$-lattices. Then $L$ is weakly injective if and only if its contragredient $L^{\prime}$ is projective.

4. Higman ideal. Let $\Lambda$ be an $R$-order, where $R$ is any noetherian domain. A $\Lambda$-bimodule is a two-sided $\Lambda$-module $T$ such that

$$
x(t y)=(x t) y, \quad \alpha t=t \alpha, \quad x, y \in \Lambda, t \in T, \alpha \in R .
$$

A 1-cocycle is a map $F \in \operatorname{Hom}_{R}(\Lambda, T)$ such that

$$
F_{x y}=x F_{y}+F_{x} y, \quad x, y \in \Lambda .
$$

For each $t_{0} \in T$, the map $F$ given by

$$
F_{x}=x t_{0}-t_{0} x, \quad x \in \Lambda
$$

is a 1-coboundary. The 1-cohomology group $H^{1}(\Lambda, T)$ is defined by

$$
H^{1}(\Lambda, T)=1 \text {-cocycles/1-coboundaries. }
$$

For example, if $N$ and $L$ are $\Lambda$-lattices, then $T=\operatorname{Hom}_{R}(N, L)$ is naturally a $\Lambda$-bimodule; and if $N$ is $R$-projective, then

$$
H^{1}(\Lambda, T)=\operatorname{Ext}_{\Lambda}^{1}(N, L) .
$$

Given a finite group $G$ of order $g$, a version of Maschke's theorem (see Zassenhaus [263], CR 73.22) asserts that

$$
g \cdot H^{1}(R G, T)=0
$$

for all $R G$-bimodules $T$. To generalize this result to an arbitrary $R$-order $\Lambda$, we begin by setting

$$
i(\Lambda)=\bigcap_{T} \operatorname{ann}_{R} H^{1}(\Lambda, T),
$$


where $T$ ranges over all $\Lambda$-bimodules, and $\mathrm{ann}_{R}$ denotes annihilator in $R$. Then $i(\Lambda)$ is an ideal in $R$, called the Higman ideal of $\Lambda$. (See D. G. Higman [118], [119], CR $\$ 75$, for the results in this section.)

Recall that the algebra $A$ is called separable over $F$ if and only if any one of the following equivalent conditions holds:

(i) For each field $E$ containing $F$, the algebra $F \otimes_{E} A$ is semisimple.

(ii) The algebra $A$ is semisimple, and the centers of its simple components are separable field extensions of $F$.

(iii) There exists a nondegenerate bilinear form $f: A \times A \rightarrow F$ satisfying $f(a b, c)=f(a, b c), a, b, c \in A$, and such that if $\left\{a_{1}, \cdots, a_{n}\right\}$ and $\left\{b_{1}, \cdots, b_{n}\right\}$ are $F$-bases of $A$ for which $f\left(a_{i}, b_{j}\right)=\delta_{i j}$, then for some $x \in A$ we have

$$
1=\sum_{1}^{n} b_{i} x a_{i}
$$

(4.1). Theorem. The ideal $i(\Lambda)$ is nonzero if and only if $A$ is separable over $F$. For any maximal ideal $P$ of $R$,

$$
i\left(\Lambda_{P}\right)=\{i(\Lambda)\}_{P}, \quad i\left(\Lambda_{P}^{*}\right)=i\left(\Lambda_{P}\right)^{*} \text {. }
$$

If $G$ is a finite group of order $g$, then $i(R G)=g R$.

Suppose now that $A$ is separable over $F$, and that $\Lambda$ is $R$-projective (the latter surely holds when $R$ is a Dedekind domain). Starting with the bilinear form $f$ in (iii) above, we may compute $i(\Lambda)$ as follows. Define

$$
\begin{aligned}
\text { inverse different } & =I(\Lambda)=\{a \in A: f(\Lambda, a) \subset R\}, \\
\text { different } & =D(\Lambda)=\{x \in A: I(\Lambda) \cdot x \subset \Lambda\}
\end{aligned}
$$

(4.2). Theorem. An element $\alpha$ of $R$ lies in $i(\Lambda)$ if and only if $\alpha$ is expressible in the form

$$
\alpha=\sum_{i=1}^{n} b_{i} x a_{i}
$$

for some $x \in D(\Lambda)$, where the $\left\{a_{i}\right\},\left\{b_{i}\right\}$ are as in (iii) above.

(For generalizations of the concept of the different, see Fossum [84], Watanabe [258], [259].)

Suppose that $\Lambda$ is a hereditary order in the semisimple algebra $A$; then submodules of free $\Lambda$-modules are projective (Cartan-Eilenberg [40, Chapter I, §5]). Since each $\Lambda$-lattice is embeddable in a free 
$\Lambda$-module, each $\Lambda$-lattice is projective. Thus for such an order $\Lambda$, we have $\operatorname{Ext}_{\Lambda}^{1}(N, L)=0$ for every pair of $\Lambda$-lattices $N, L$. On the other hand, it may well happen that the Higman ideal $i(\Lambda)$ is a proper ideal of $R$, and that $H^{1}(\Lambda, T) \neq 0$ for some $T$. Since representation theory is more concerned with extensions of modules than with cohomology of bimodules, it is of interest to investigate the $R$-annihilator of Ext. In this direction we have

(4.3). Theorem. Let $R$ be a Dedekind domain of characteristic zero, $G$ a finite group of order $g, M$ an RG-lattice of R-rank $m$. Suppose that FM is an absolutely irreducible $F G$-module (that is, $\left.\operatorname{Hom}_{F G}(F M, F M) \cong F\right)$. Let $\alpha \in R$. Then

$$
\alpha \cdot \operatorname{Ext}_{R G}^{1}(M, N)=0
$$

for every RG-lattice $N$ if and only if $\alpha \in(\mathrm{g} / \mathrm{m}) R$. Dually, if $F N$ is absolutely irreducible, then (4.4) holds for each $R G$-lattice $M$ if and only if $\alpha \in(g / n) R$, where $n$ is the R-rank of $N$.

References. Reiner [189]; see also D. G. Higman [120], [121].

This result has been generalized by Jacobinski [125] and Roggenkamp [208a]. Let $R=$ alg. int. $\{F\}$, and set

$$
\begin{aligned}
F G & =\sum^{\cdot} A_{i} \text { (simple components), } \\
F_{i} & =\text { center of } A_{i}, R_{i}=\text { alg. int. }\left\{F_{i}\right\}, \quad\left(A_{i}: F_{i}\right)=n_{i}^{2} .
\end{aligned}
$$

Let $D_{i}^{-1}$ be the inverse different of $R_{i}$ with respect to $R$, relative to the ordinary trace form from $F_{i}$ to $F$. Define the integral ideal $d_{i}$ of $R$ by setting $d_{i}^{-1}=D_{i}^{-1} \cap F$.

Now let $\Lambda_{0}$ be any maximal order of $F G$ containing $R G$. Since $\Lambda_{0}$ is a hereditary ring (see $\S 7$ ), $\operatorname{Ext}_{\Lambda_{0}}^{1}\left(N_{0}, L_{0}\right)=0$ for each pair of $\Lambda_{0}$-lattices $N_{0}, L_{0}$. Using this fact, together with arithmetic properties of maximal orders, one obtains

(4.5). THEOREM. Let $e_{i}$ be a primitive central idempotent of $F G$.

(i) $\left(g / n_{i}\right) d_{i}^{-1}$ is an integral ideal of $R$.

(ii) If $M, N$ are $R G$-lattices for which either $e_{i} M=M$ or $e_{i} N=N$, then

$$
\left(g / n_{i}\right) d_{i}^{-1} \cdot \operatorname{Ext}_{R G}^{1}(M, N)=0 .
$$

(iii) Let $M$ be an $R G$-lattice such that $e_{i} M=M$, and let $\alpha \in R$ have the property that $\alpha \cdot \operatorname{Ext}_{R G}^{1}(M, N)=0$ for every $R G$-lattice $N$. Then $\alpha \in\left(g / n_{i}\right) d_{i}^{-1}$.

References. Parts (i), (ii) are due to Jacobinski [126], and (iii) was proved by Roggenkamp [208a]. 
We quote some miscellaneous results on the vanishing of Ext.

(4.6). TheOREM (Berman [21]). Suppose that $G$ has a normal cyclic Sylow p-subgroup, and let $M, N$ be $Z_{p}^{*} G$-lattices such that $Q_{p}^{*} M \cong Q_{p}^{*} N$. Then

$$
\operatorname{Ext}_{Z_{p}^{*}}^{1}(M, N)=0
$$

Gudivok [100] remarks that this no longer holds if in place of $Z_{p}^{*}$ we use $R_{P}^{*}$, where $R=$ alg. int. $\{F\}$.

Berman-Lihtman [30], generalized by Reiner [194], discuss the question as to when $\operatorname{Ext}_{R G}^{1}(M, N)=0$ for all $R G$-lattices $M, N$ such that $F M, F N$ are a fixed pair of $F G$-modules.

5. Representations over local domains. Throughout this section let $R$ be a discrete valuation ring with quotient field $F$, maximal ideal $P$, residue class field $\bar{R}=R / P$. Let $R^{*}$ be the $P$-adic completion of $R$, with quotient field $F^{*}$. For any $R$-lattice $M$, let $M^{*}=R^{*} M$, $\bar{M}=M / P M \cong M^{*} / P^{*} M^{*}$. Now let $\Lambda^{*}$ be any $R^{*}$-order; then $\bar{\Lambda}$ $=\Lambda^{*} / P^{*} \Lambda^{*}$ is a finite dimensional $\bar{R}$-algebra. If $e \in \Lambda^{*}$ is idempotent, so also is $\vec{e}$. A fundamental result is

(5.1). Theorem on Lifting IDEmpotents. Every idempotent in $\bar{\Lambda}$ is of the form e for some idempotent $e \in \Lambda^{*}$.

References. CR $\$ 77$ for this specific case. A more general discussion of the problem is given in Jacobson [132, Chapter III, §8].

This theorem is a generalization of Hensel's Lemma; its validity means that $P$-adic methods will play as large a role in integral representation theory as they already do in algebraic number theory. The most important consequence of (5.1) is

(5.2). KRUll-Schmidt Theorem. Every $\Lambda^{*}$-lattice is a finite direct sum of indecomposable lattices, which are uniquely determined up to $\Lambda^{*}$-isomorphism and order of occurrence.

References. The result, due to Azumaya [3], was rediscovered by various authors; see Borevič-Faddeev [32, II], Reiner [186], [193], Swan [232].

The key step in the proof is

(5.3). Proposition. Let $M$ be a $\Lambda^{*}$-lattice, and set $E(M)$ $=\operatorname{Hom}_{\Lambda^{*}}(M, M), \hat{E}(M)=E(M) / \operatorname{rad} E(M)$. Then $M$ is indecomposable if and only if $\hat{E}(M)$ is a skewfield.

(5.4). Corollary (Reiner [193]). Let $M$ be a $\Lambda^{*}$-lattice; call $M$ 
absolutely indecomposable if $M$ remains indecomposable under all ground ring extensions. Then $M$ is absolutely indecomposable if and only if $\hat{E}(M)$ is a field which is purely inseparable over $\bar{R}$.

Suppose now that $\Lambda$ is an $R$-order in the separable $F$-algebra $A$, and let $i(\Lambda)$ be the Higman ideal of $\Lambda$. Then $i(\Lambda)=P^{k_{0}}$ for some $k_{0}$, and by (4.1), $i\left(\Lambda^{*}\right)=\left(P^{*}\right)^{k_{0}}$. For a $\Lambda$-lattice $M$, the $\Lambda$-module $M / P^{k} M$ will be denoted by $\langle M\rangle_{k}$, and a similar notation will be used for $\Lambda^{*}$-lattices.

(5.5). Lemma. Let $M, N$ be $\Lambda$-lattices, and let $k>k_{0}$. Given any $f \in \operatorname{Hom}_{\Lambda}\left(\langle M\rangle_{k},\langle N\rangle_{k}\right)$, there exists a map $F \in \operatorname{Hom}_{\Lambda}(M, N)$ such that $f$ and $F$ induce the same map from $\langle M\rangle_{k-k_{0}}$ into $\langle N\rangle_{k-k_{0}}$.

References. Maranda [154], D. G. Higman [120], [121], NazarovaRoĭter [169].

Using the preceding lemma, one obtains

(5.6). Theorem. Let $M, N$ be $\Lambda$-lattices, $X$ a $\Lambda^{*}$-lattice.

(i) If $M \cong N$ then $\langle M\rangle_{k} \cong\langle N\rangle_{k}$ for all $k \geqq 0$.

(ii) If $\langle M\rangle_{k} \cong\langle N\rangle_{k}$ for some $k>k_{0}$, then $M \cong N$.

(iii) $M \cong N$ if and only if $M^{*} \cong N^{*}$.

(iv) If $\langle X\rangle_{k}$ is decomposable for some $k>k_{0}$, then $X$ is decomposable.

(v) Suppose that for some $k>2 k_{0},\langle X\rangle_{k}$ contains a $\Lambda^{*}$-submodule $U$

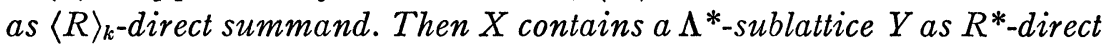
summand, and $Y$ coincides with $U$ modulo $\left(P^{*}\right)^{k-k_{0}}$.

(vi) If $k_{0}=0$, then $M \cong N$ if and only if $F M \cong F N$.

References. These results are originally due to Maranda [154], with a somewhat weaker version of (iv). Improvements and generalizations are given in Heller [109], D. G. Higman [121], CR $\$ 76$.

The passage from $\Lambda^{*}$-lattices to $\Lambda$-lattices is facilitated by

(5.7). Proposition. Let $L$ be a $\Lambda^{*}$-lattice, $V$ an $A$-module such that $F^{*} L=F^{*} V$. Set $M=L \cap V$; then $M$ is a $\Lambda$-lattice for which $M^{*}=L$, $F M=V$.

References. Bourbaki [36, Chapter 3, §3, no. 5]; Heller [109]; Takahashi [238]; CR \$76.

(5.8). Corollary. If $A$ is a direct sum of full matrix algebras over $F$, then every $\Lambda^{*}$-lattice is of the form $R^{*} M$ for some $\Lambda$-lattice $M$. The Krull-Schmidt Theorem holds for $\Lambda$-lattices. Every idempotent in $\bar{\Lambda}$ is of the form $\bar{e}$ for some idempotent $e \in \Lambda$.

REMARK. See Heller [109], CR \$76. The same conclusions are 
valid if we merely assume that $A$ is a direct sum of full matrix algebras over skewfields $S_{i}$ such that for each $i, S_{i} \otimes_{F^{*}} F$ is also a skewfield.

(5.9). Theorem (Reiner [186]). Let $L, M, N$ be $\Lambda$-lattices. Then

(i) $M^{(t)} \cong N^{(t)}$ if and only if $M \cong N$.

(ii) $M \oplus L \cong N \oplus L$ if and only if $M \cong N$.

(5.10). Theorem. Let $F^{\prime}$ be a finite separable extension of $F$, and let $R^{\prime}$ be a valuation ring in $F^{\prime}$ containing $R$. For $M$ any $\Lambda$-lattice, define

$$
M^{\prime}=R^{\prime} \otimes_{R} M, \quad \Lambda^{\prime}=R^{\prime} \otimes_{R} \Lambda,
$$

so that $M^{\prime}$ is a $\Lambda^{\prime}$-lattice. Then for any pair of $\Lambda$-lattices $M, N$ we have $M \cong N$ if and only if $M^{\prime} \cong N^{\prime}$.

References. Reiner-Zassenhaus [199]. See Bialnicki-Birula [31a] for generalization.

6. Genus. Throughout this section let $A$ be a semisimple algebra over the algebraic number field $F$, and let $R$ be any Dedekind domain with quotient field $F$. Let $\Lambda$ be an $R$-order in $A$. By (4.1) the Higman ideal $i(\Lambda)$ is a nonzero ideal in $R$, and $i(\Lambda)$ annihilates $H^{1}(\Lambda, T)$ for every $\Lambda$-bimodule $T$. Let $M, N$ be any $\Lambda$-lattices; since $R$ is a Dedekind domain, from (1.2) we see that $M$ is $R$-projective. The discussion in $\$ 3$ then yields

$$
\operatorname{Ext}_{\Lambda}^{1}(M, N)=H^{1}\left(\Lambda, \operatorname{Hom}_{R}(M, N)\right),
$$

and consequently

$$
i(\Lambda) \cdot \operatorname{Ext}_{\Lambda}^{1}(M, N)=0 \quad \text { for any } \Lambda \text {-lattices } M, N .
$$

We have denoted by $R_{P}$ the localization of $R$ at its maximal ideal $P$, and by $R_{P}^{*}$ the $P$-adic completion of $R$. Two $\Lambda$-lattices $M, N$ are in the same genus (notation: $M \bigvee N$ ) if

$$
F M \cong F N, \quad M_{P} \cong N_{P} \text { for each } P .
$$

Denote by $\Gamma(M)$ the set of all $\Lambda$-lattices in the same genus as $M$. Since $F M \cong F N$ implies that $M_{P} \cong N_{P}$ whenever $P D i(\Lambda)$ (see (5.6)), in condition (6.2) it suffices to let $P$ range over the maximal ideals of $R$ containing $i(\Lambda)$. The requirement $F M \cong F N$ is usually superfluous, and is inserted here for emphasis, since in fact if $M_{P} \cong N_{P}$ for even one $P$, then automatically $F M \cong F N$. We remark also that by (5.6), $M_{P} \cong N_{P}$ if and only if $M_{P}^{*} \cong N_{P}^{*}$.

(Instead of using the Higman ideal, it is sometimes more conveni- 
ent to introduce the finite set $J$ of all maximal ideals $P$ of $R$ for which $\Lambda_{P}$ is not a maximal $R_{P}$-order in $A$. In that case, $M \bigvee N$ if and only if $F M \cong F N$ and $M_{P} \cong N_{P}, P \in J$. See (7.6).)

Let us introduce the semilocal Dedekind domain $\widetilde{R}$ defined by

$$
\widetilde{R}=\bigcap_{P \supset i(\Lambda)} R_{P}
$$

Of course $F$ is also the quotient field of $\tilde{R}$. We set $\tilde{\Lambda}=\widetilde{R} \Lambda, \tilde{M}=\widetilde{R} M$, and so on, so $\tilde{M}$ is a $\tilde{\Lambda}$-lattice.

(6.3). Theorem. Let $M, N$ be $\Lambda$-lattices.

(i) $M \vee N$ if and only if $\tilde{M} \cong \tilde{N}$.

(ii) $\operatorname{Ext}_{\Lambda}^{1}(M, N) \cong \operatorname{Ext}_{\tilde{\Lambda}}^{1}(\tilde{M}, \tilde{N})$.

(iii) $M$ is decomposable if and only if $\tilde{M}$ is decomposable.

(iv) $M \bigvee N$ if and only if for every nonzero ideal $J$ in $R$, there exists a $\Lambda$-monomorphism $\phi: M \rightarrow N$ such that $N / \phi(M)$ is an R-torsion module whose $R$-annihilator is relatively prime to $J$. (In particular, if $\phi$ can be chosen so that $\operatorname{ann}_{R}(N / \phi(M))$ is relatively prime to $i(\Lambda)$, then $M \vee N$.

References. CR §81, Maranda [153], Reiner [188], Takahashi [238].

The following generalization of the "strong approximation theorem" of algebraic number theory is often useful.

(6.4). Theorem. Let $W$ be any A-module. Let $s$ be some finite set of maximal ideals of $R$, and suppose that for each $P \in \mathcal{S}$ we are given a $\Lambda_{P}^{*}$-lattice $X^{(P)}$ such that

$$
F_{P}^{*} \otimes_{R_{P}^{*}} X^{(P)} \cong F_{P}^{*} \otimes_{F} W
$$

as $A_{P}^{*}$-modules. Then there exists a $\Lambda$-lattice $M$ for which $F M \cong W$ and

$$
R_{P}^{*} \otimes_{R} M \cong X^{(P)}, \quad P \in \mathcal{S}
$$

References. Bourbaki [36, Chapter 7], Takahashi [238].

For an $A$-module $W$, we have denoted by $\sigma(W)$ the collection of $\Lambda$-lattices $M$ such that $F M \cong W$. If $M, N \in \sigma(W)$, we call $M$ and $N$ $R_{P}$-equivalent if $M_{P} \cong N_{P}$.

(6.5). Theorem (Maranda [153], Takahashi [238]). The number of genera in $\sigma(W)$ equals the number of $\tilde{\Lambda}$-isomorphism classes in $\sigma(W)$, and this number is given by 


$$
\prod_{P \supset i(\Lambda)} h_{P}
$$

where $h_{P}$ is the number of classes in $\sigma(W)$ relative to $R_{P}$-equivalence.

To determine the number of $\Lambda$-isomorphism classes in $\sigma(W)$, we may first use (6.5) to count the number of genera in $\sigma(W)$. Then we are left with the question of finding the number $|\Gamma(M)|$ of $\Lambda$-isomorphism classes in the genus $\Gamma(M)$ containing a $\Lambda$-lattice $M$. It may happen that $|\Gamma(M)|$ is not the same for each $M \in \sigma(W)$; see for example Nazarova-Rointer [167] for the case where $\Lambda=Z G, G=S_{3}$.

The $A$-module $W$ is called absolutely irreducible if $W$ is irreducible, and remains irreducible under arbitrary extension of the ground field. Since $A$ is semisimple, $W$ is absolutely irreducible if and only if $\operatorname{Hom}_{A}(W, W) \cong F$.

(6.6). Theorem (Maranda [153], TAKahashi [238]). Let $M$ be a $\Lambda$-lattice such that $F M$ is absolutely irreducible. Then each $N \in \Gamma(M)$ is isomorphic to a $\Lambda$-lattice $J M$ for some nonzero $R$-ideal $J$ in $F$. Further, $J_{1} M \cong J_{2} M$ if and only if $J_{2}=J_{1} \alpha$ for some $\alpha \in F$. Hence $|\Gamma(M)|$ equals the number of ideal classes in $R$.

In studying questions of decomposability, it is convenient to introduce the following concept: let $M, N$ be $\Lambda$-lattices, and call $N$ a local summand of $M$ if for each maximal ideal $P$ of $R, N_{P}^{*}$ is isomorphic to a $\Lambda_{P}^{*}$-direct summand of $M_{P}^{*}$. (It suffices to assume this condition for those $P$ which contain $i(\Lambda)$; if $i(\Lambda)=R$, assume instead that $F N$ is isomorphic to a direct summand of $F M$.)

(6.7). THEorem. Let $M, N$ be $\Lambda$-lattices such that $N$ is a local summand of $M$. Then there exists a decomposition $M=X \oplus Y$ into $\Lambda$-lattices, with $X \in \Gamma(N)$.

References. Jones [136], Jacobinski [128], Reiner [188].

A much deeper result of this nature is

(6.8). Theorem (Jacobinski [129]). Let $M, N$ be $\Lambda$-lattices such that $N$ is a local summand of $M$. Assume that every irreducible $A$ module appearing as a composition factor of $F N$ appears with greater multiplicity in FM. Then there exists a decomposition $M \cong N \oplus Y$ for some $\Lambda$-lattice $Y$.

(6.9). Corollary. Let $M, N$ be $\Lambda$-lattices in the same genus. Then $N$ is isomorphic to a direct summand of $M+M$.

Call a $\Lambda$-lattice $T$ faithful if no nonzero element of $\Lambda$ annihilates $T$. 
The following elegant result was proved by Router [212], [217], and is essentially equivalent to Theorem 6.8.

(6.10). Theorem. Let $M, N$ be $\Lambda$-lattices in the same genus, and let $T$ be any faithful $\Lambda$-lattice. Then there exists a $\Lambda$-lattice $T^{\prime} \in \Gamma(T)$ such that $M+T \cong N+T^{\prime}$.

As a consequence of this result, it is possible to prove

(6.11). Theorem (Roiter [212], JACOBINSKi [129]). Let $\Lambda$ be an $R$-order. There exists a positive integer $b$ depending only on $\Lambda$, such that $|\Gamma(M)| \leqq b$ for every $\Lambda$-lattice $M$.

We shall return to the study of genera in $\S 8$. Further references on genera are Faddeev [79], [80], [82] (the first of these articles is semiexpository), Drozd-Turčin [75], Jacobinski [128], [130], Roŭter [210]-[214], Drozd [70a], [706], Roggenkamp [205].

7. Maximal orders. The importance of the theory of maximal orders in integral representations has become increasingly evident from the work of Heller-Reiner [115], Jacobinski [126], [128], [129], and Swan [234]. We devote this section to a summary of the main results of the theory, and refer the reader to the following references: Auslander-Goldman [1], Chevalley [41], Deuring [56], RoggenkampDyson [208b], Jacobson [131], Reiner [198], Schilling [222], Weil [260].

Assume throughout that $R$ is a Dedekind domain with quotient field $F$. (The more general case where $R$ is a noetherian integrally closed domain is treated in Auslander-Goldman [1]; see also Fossum [84a].) Let $A$ be a finite dimensional $F$-algebra; an element $x \in A$ is integral over $R$ if $x$ is a zero of a monic polynomial with coefficients in $R$. The set of all such elements $x$ is the integral closure of $R$ in $A$, and is a ring when $A$ is commutative.

If $A$ is a simple algebra with center $F$, we may choose an extension field $E$ of $F$ for which $E \otimes_{F} A \cong(E)_{m}$. Then each $x \in A$ maps onto $1 \otimes x \in E \otimes A$, and is therefore representable as a matrix $M(x) \in(E)_{m}$. The characteristic polynomial

$$
\operatorname{det}(\lambda I-M(x))=\lambda^{m}-T(x) \lambda^{m-1}+\cdots+(-1)^{m} N(x)
$$

has coefficients in $F$, and is called the reduced characteristic polynomial of $x$. Call $T(x)$ the reduced trace of $x$, and $N(x)$ the reduced norm of $x$. Then $T: A \rightarrow F$ is an $F$-linear map, while $N: A \rightarrow F$ is multiplicative.

Returning to the general case of an arbitrary $F$-algebra $A$, we call $J$ a full $R$-lattice in $A$ if $J$ is an $R$-lattice for which $F \cdot J=A$. For such a lattice, define 


$$
\begin{aligned}
\text { left order of } J & =O_{l}(J)=\{x \in A: x J \subset J\}, \\
\text { right order of } J=O_{r}(J) & =\{x \in A: J x \subset J\} .
\end{aligned}
$$

These are $R$-orders in $J$, and $J$ is a left $O_{l}(J)$-lattice and a right $O_{r}(J)$-lattice. Put

$$
J^{-1}=\{x \in A: J x J \subset J\}=\left\{x \in A: J x \subset O_{l}(J)\right\},
$$

again a full $R$-lattice in $A$, called the inverse of $J$.

An $R$-order in $A$ is a maximal $R$-order if it is not properly contained in any bigger $R$-order in $A$.

(7.1). Theorem. If $A$ is a separable $F$-algebra, ${ }^{3}$ every $R$-order in $A$ is contained in a maximal $R$-order.

(7.2). Theorem. Let $A$ be a separable F-algebra, and suppose that $A=A_{1} \oplus \cdots \oplus A_{r}$ (simple components). Let $F_{i}$ be the center of $A_{i}$, and $R_{i}=$ integral closure of $R$ in $F_{i}(1 \leqq i \leqq r)$. Then the maximal $R-$ orders $\Lambda$ in $A$ are precisely those orders of the form $\Lambda_{1} \oplus \cdots \oplus \Lambda_{r}$, where each $\Lambda_{i}$ is a maximal $R$-order in $A_{i}$. Further, maximal $R$-orders in $A_{i}$ coincide with maximal $R_{i}$-orders in $A_{i}$. Every element of an $R$-order in $A$ is integral over $R$. When $A$ is commutative, $R_{1} \oplus \cdots \oplus R_{r}$ is the unique maximal $R$-order in $A$.

For the remainder of this section, assume that $A$ is a separable $F$-algebra. The preceding theorem shows that the study of maximal orders can always be reduced to the central simple case. However, for the time being, we shall not make such a reduction.

(7.3). Theorem. Let $\Lambda$ be any $R$-order in $A$, and let $P$ range over the maximal ideals of $R$. The following statements are equivalent:

(i) $\Lambda$ is a maximal $R$-order in $A$.

(ii) For each $P, \Lambda_{P}$ is a maximal $R_{P}$-order in $A$.

(iii) For each $P, \Lambda_{P}^{*}$ is a maximal $R_{P}^{*}$-order in $A_{P}^{*}$.

REMARK. For each fixed $P$, (ii) and (iii) are equivalent.

In the case of central simple algebras, we have

(7.4). Theorem. Let $\Lambda_{P}$ be an $R_{P}$-order in the simple algebra $A$ with center $F$. Then $\Lambda_{P}$ is a maximal $R_{P}$-order if and only if $\Lambda_{P}$ is a left hereditary ring whose Jacobson radical $\operatorname{rad} \Lambda_{P}$ is the unique maximal two-sided ideal of $\Lambda_{P}$.

${ }^{3}$ See $\$ 4$. When char $F=0$, this merely means that $A$ is semisimple. Without some hypothesis on $A$, maximal orders need not exist (see Deuring [56], Faddeev [80]). 
(7.5). ThEOREM. Let $\Lambda_{P}$ be a maximal $R_{P}$-order in the central simple F-algebra $A$. Then:

(i) $\operatorname{rad} \Lambda_{P} \supset P \cdot \Lambda_{P}$, and $\Lambda_{P} / \operatorname{rad} \Lambda_{P} \cong \Lambda_{P}^{*} / \operatorname{rad} \Lambda_{P}^{*}=\operatorname{simple}(R / P)$ algebra.

(ii) Every full two-sided ideal in $\Lambda_{P}$ is a power of $\operatorname{rad} \Lambda_{P}$.

(iii) Every one-sided ideal in $\Lambda_{P}$ (full or not) is principal.

(iv) Two $\Lambda_{P}$-lattices $M, N$ are isomorphic if and only if $F M \cong F N$.

(v) The maximal $R_{P}$-orders in $A$ are precisely the orders of the form $t^{-1} \Lambda_{P} t, t=$ unit in $A$.

As a consequence of (7.2) and (7.5), we obtain

(7.6). Theorem. Let $A$ be any separable algebra over $F$. Then every maximal $R$-order $\Lambda$ in $A$ is left and right hereditary, and every $\Lambda$-lattice is projective. If $M$ and $N$ are $\Lambda$-lattices such that $F M \cong F N$, then $M_{P} \cong N_{P}$ for each maximal ideal $P$ of $R$.

REMARK. There exist hereditary orders which are not maximal; see Brumer [39], Harada [104], [105], and also $\$ 13$ of this article.

We turn next to the multiplicative theory of ideals in maximal orders. The first basic result is

(7.7). Theorem. Let $J$ be a full $R$-lattice in $A$, where $A$ is any separable $F$-algebra. Then $O_{l}(J)$ is a maximal order if and only if $O_{r}(J)$ is a maximal order.

A normal ideal in $A$ is a full $R$-lattice $J$ in $A$ both of whose orders $O_{l}(J), O_{r}(J)$ are maximal. Call $J$ integral if $J \subset O_{l}(J)$, or equivalently, if $J \subset O_{r}(J)$. If $J$ is a normal ideal, so is $J^{-1}$, and the following relations are valid:

$O_{r}\left(J^{-1}\right)=O_{l}(J), \quad O_{l}\left(J^{-1}\right)=O_{r}(J), \quad J \cdot J^{-1}=O_{l}(J), \quad J^{-1} \cdot J=O_{r}(J)$

Let $\Lambda$ be a maximal $R$-order in the separable $F$-algebra $A$. By a $\Lambda$-ideal in $A$ we shall mean a full $R$-lattice $J$ in $A$ for which $O_{l}(J)$ $=O_{r}(J)=\Lambda$. A prime ideal of $\Lambda$ is a maximal $\Lambda$-ideal contained in $\Lambda$. Equivalently, a prime ideal is an integral $\Lambda$-ideal $\mathfrak{P}$ such that whenever $\mathfrak{B}$ contains the product of two integral $\Lambda$-ideals, it must contain one of the factors. If $\mathfrak{P}$ is a prime ideal of $\Lambda$, then $\Lambda / \mathfrak{B}$ is a simple algebra over the field $R / P$, where $P=R \cap \mathfrak{B}$.

(7.8). THEOREM. The set $I\{\Lambda\}$ of $\Lambda$-ideals in $A$ is an abelian group relative to the multiplication $J \cdot J^{\prime}$ of $\Lambda$-ideals. The identity element of the group is $\Lambda$ itself, and the inverse of $J$ is $J^{-1}$. Further, $I\{\Lambda\}$ is the free abelian group generated by the prime ideals of $\Lambda$. 
If $A$ is given as in (7.2), and $\Lambda=\Lambda_{1} \oplus \cdots \oplus \Lambda_{r}$, then the prime ideals of $\Lambda$ are obtained by replacing exactly one summand $\Lambda_{i}$ by one of its prime ideals $\mathfrak{B}$. Furthermore, for each $\Lambda_{i}$ there is a one-to-one correspondence $\mathfrak{B} \leftrightarrow R_{i} \cap \mathfrak{B}$ between the prime ideals $\mathfrak{B}$ of $\Lambda_{i}$ and the maximal ideals $R_{i} \cap \mathfrak{B}$ of $R_{i}$. We discuss this in more detail below (see (7.13)).

While the theory of (two-sided) $\Lambda$-ideals in $A$ is not too hard, that of one-sided ideals in $\Lambda$ is more complicated, since together with $\Lambda$ one must also consider other maximal orders as well. A product $J_{1} J_{2} \cdots J_{n}$ of normal ideals in $A$ is called proper if $O_{r}\left(J_{i}\right)=O_{l}\left(J_{i+1}\right)$, $1 \leqq i \leqq n-1$. This product is again a normal ideal, with left order $O_{l}\left(J_{1}\right)$ and right order $O_{r}\left(J_{n}\right)$.

(7.9). Theorem. Let $J, J^{\prime}$ be normal ideals with $J \supset J^{\prime}$. Then there exist normal integral ideals $N_{1}, N_{2}$ such that $J^{\prime}$ is a proper product $N_{1} J N_{2}$. If $J$ and $J^{\prime}$ have the same left order, the factor $N_{1}$ may be omitted.

A maximal integral ideal ${ }^{4}$ is a normal ideal $\mathfrak{M}$ which is a maximal left ideal in its left order $\Lambda$ (or equivalently, a maximal right ideal in its right order $\Lambda^{\prime}$ ). The largest $\Lambda$-ideal $\mathfrak{P}$ contained in $\mathfrak{M}$ is a prime ideal of $\Lambda$, and $\Lambda / \mathfrak{M}$ is an irreducible module for the simple algebra $\Lambda / \mathfrak{F}$. We may characterize $\mathfrak{B}$ as the $\Lambda$-annihilator of $\Lambda / \mathfrak{M}$.

If $\Lambda$ and $\Lambda^{\prime}$ are maximal orders in $A$, we may choose a normal ideal $J_{0}$ with left order $\Lambda$, right order $\Lambda^{\prime}$. The map $N \rightarrow J_{0}^{-1} N J_{0}, N \in I\{\Lambda\}$, gives an isomorphism $I\{\Lambda\} \cong I\left\{\Lambda^{\prime}\right\}$. This isomorphism is independent of the choice of $J_{0}$. The normal ideals $N$ and $J_{0}^{-1} N J_{0}$ are said to be similar.

(7.10). THEOREM. Let $J$ be any integral normal ideal properly contained in its left order $\Lambda$. Then $J$ is expressible as a proper product of maximal integral ideals $\mathfrak{M}_{1}, \cdots, \mathfrak{M}_{l}$ :

$$
J=\mathfrak{M}_{1} \cdots \mathfrak{M}_{l}
$$

The number $l$ equals the number of composition factors of the left $\Lambda$-module $\Lambda / J$. The prime ideals associated with the $\left\{\mathfrak{M}_{i}\right\}$ are similar to the $\Lambda$-annihilators of the composition factors of $\Lambda / J$, and hence are uniquely determined by $J$ up to similarity and order of occurrence. Finally, the set of integral normal ideals in $A$ forms a groupoid ${ }^{5}$ relative to proper products.

"Some authors use the term "indecomposable ideal" instead; this leads to some confusion when one studies lattices, and so we propose the clearer terminology "maximal integral ideal."

${ }^{5}$ This is Brandt's groupoid; see Jacobson [131, p. 132]. 
For the remainder of this section we shall concentrate our attention on the study of maximal orders in central simple algebras. Let $A$ be a simple algebra with center $F$, and say $A \cong(D)_{n}$ where $D$ is a skewfield with center $F$. We call $((D: F))^{1 / 2}$ the index of $A$. Let $W$ be an irreducible left $A$-module, viewed as right $D$-space of dimension $n$. Then

$$
A=\operatorname{Hom}_{D}(W, W), \quad D=\operatorname{Hom}_{A}(W, W) .
$$

We begin with a pair of theorems relating the study of maximal orders in $A$ with those in $D$.

(7.12). Theorem. Let $R$ be a Dedekind domain with quotient field $F$, and let $\Delta$ be some fixed maximal $R$-order in $D$. Let $M$ be any full right $\Delta$-lattice in $W$, and put $\Lambda=\operatorname{Hom}_{\Delta}(M, M)$. Then $\Lambda$ is a maximal $R$-order in $A$. Every other maximal $R$-order in $A$ is of the form $\operatorname{Hom}_{\Delta}(N, N)$ for some full right $\Delta$-lattice $N$ in $W$.

Furthermore there is a one-to-one inclusion-preserving correspondence (the Morita correspondence) $X \leftrightarrow L$ between the set of all left $\Lambda$-lattices $X$ and the set of all left $\Delta$-lattices $L$, with

$$
X=M \otimes_{\Delta} L, \quad L=\tilde{M} \otimes_{\Lambda} X .
$$

Here, $\tilde{M}=\operatorname{Hom}_{\Delta}(M, \Delta)$ is viewed as left $\Delta$-, right $\Lambda$-lattice.

We may embed $D$ in $A$ by identifying each element $d \in D$ with a diagonal matrix all of whose diagonal entries are equal to $d$. Keeping the notation of the preceding theorem, we have

(7.13). Theorem. To each maximal ideal $P$ of $R$ there corresponds a unique prime ideal $\mathfrak{p}$ of $\Delta$ containing $P$, and

$$
P=\mathfrak{p} \cap R, \quad P \Delta=\mathfrak{p}^{e} \text { for some } e .
$$

Set $\mathfrak{P}=\mathfrak{p} \Lambda$; then $\mathfrak{P}$ is the unique prime ideal of $\Lambda$ containing $P$, and

$$
P=\mathfrak{P} \cap R, \quad P \Lambda=\mathfrak{P}^{e}, \quad \Lambda / \mathfrak{P} \cong(\Delta / \mathfrak{p})_{n} .
$$

Both $\Lambda / \mathfrak{P}$ and $\Delta / \mathfrak{p}$ are simple $(R / P)$-algebras.

Further, the group $I\{\Lambda\}$ of $\Lambda$-ideals in $A$ is free abelian on the set of generators $\mathfrak{B}$, one for each maximal ideal $P$ of $R$. If $\mathfrak{M}$ is any maximal left ideal of $\Lambda$, then $\mathfrak{M}$ contains a unique prime ideal $\mathfrak{P}$ of $\Lambda$, and $\mathfrak{M}$ is the inverse image under the map $\Lambda \rightarrow \Lambda / \mathfrak{B}$ of some maximal left ideal of $\Lambda / \mathfrak{P}$.

The two preceding theorems show how to reduce questions about maximal orders in simple algebras to the case of maximal orders in skewfields. Concerning these we have a basic "local" result: 
(7.14). Theorem. Let $R$ be a complete discrete valuation ring, $D$ a skewfield with center $F$. Let $N: D \rightarrow F$ be the reduced norm map. Then an element $a \in D$ is integral over $R$ if and only if $N(a) \in R$. The integral closure $\Delta$ of $R$ in $D$ is a ring, and is the unique maximal order in $D$. In $\Delta$ there is a unique maximal two-sided ideal $\mathfrak{p}$, given by

$$
\mathfrak{p}=\{a \in D: N(\alpha) \in P\} .
$$

In fact, $\mathfrak{p}$ is a maximal left ideal of $\Delta$, and every nonzero one-sided ideal of $\Delta$ is a power of $\mathfrak{p}$. The ideal $\mathfrak{p}$ is a principal left ideal, and $\Delta / \mathfrak{p}$ is a skewfield of finite dimension over $R / P$.

Suppose in particular that $R$ is the $P$-adic completion of a ring of algebraic integers, and let $m$ be the index of $D$. In that case $\Delta / p$ is a field of dimension $m$ over $R / P$, and furthermore $P \Delta=p^{m}$.

Turning now to the global case, let $A$ be a central simple $F$-algebra, where $F$ is an algebraic number field which is the quotient field of some Dedekind domain $R$. If $P$ is any maximal ideal of $R$, we may form the central simple $F_{P}^{*}$-algebra $A_{P}^{*}$; the index $m_{P}$ of $A_{P}^{*}$ is called the $P$-index of $A$. Set $A_{P}^{*} \cong(S)_{t}$, where $S$ is a skewfield of index $m_{P}$; let $\bar{S}$ denote the residue class field of the maximal $R_{P}^{*}$-order in $S$ modulo its prime ideal.

(7.15). Theorem. Let $\Lambda$ be a maximal $R$-order in the central simple $F$-algebra $A$, where $F$ is an algebraic number field. Let $P$ be a maximal ideal of $R$, and $\Re$ the corresponding prime ideal of $\Lambda$. Denote by $m_{P}$ the $P$-index of $A$. Then (using the notation above)

$$
\Lambda / \mathfrak{P} \cong(\bar{S})_{t}, \quad(\bar{S}: R / P)=m_{P}, \quad P \Lambda=\mathfrak{B}^{m_{P}} .
$$

Further, if $D$ is the different of $\Lambda$ with respect to $R$ relative to the reduced trace map from $A$ to $F$, then $\mathfrak{B}$ appears with exponent $m_{P}-1$ in $\delta$. (If $m_{P}>1$, we say that $A$ is ramified at $P$.)

Keeping the above notation, let $N: A \rightarrow F$ be the reduced norm map. For a full $R$-lattice $J$ in $A$, define its reduced norm $N(J)$ to be the $R$-ideal of $F$ generated by the set of elements $\{N(a): a \in J\}$.

(7.16). THEOREM. If $\mathfrak{M}$ is a maximal integral ideal, its reduced norm $N(\mathfrak{M})$ is the maximal ideal $\mathfrak{M} \cap R$ of $R$. If $J$ is any normal integral ideal with left order $\Lambda$, then $N(J)$ is the product of the R-annihilators of the $\Lambda$-composition factors of $\Lambda / J$. Further, if $J J^{\prime}$ is a proper product of normal ideals, then

$$
N\left(J J^{\prime}\right)=N(J) \cdot N\left(J^{\prime}\right), \quad N\left(J^{-1}\right)=N(J)^{-1} .
$$

We have denoted by $I\{R\}$ the group of $R$-ideals in $F$. Set 


$$
I^{\prime}\{R\}=\{N(a) R: a=\text { unit in } A\} .
$$

Then it is clear that the reduced norm maps the set of normal ideals in $A$ onto $I\{R\}$, carrying the set of principal ideals onto $I^{\prime}\{R\}$. The factor group $I\{R\} / I^{\prime}\{R\}$ is finite.

A prime of the algebraic number field $F$ is an equivalence class of valuations of $F$. The finite primes are those which come from $P$-adic valuations of $F$, where $P$ is a maximal ideal in alg. int. $\{F\}$. On the other hand, an infinite prime $P$ is one whose class of valuations extends the ordinary absolute value on the rational field $Q$. Let $F_{P}^{*}$ be the $P$-adic completion of $F$, where $P$ is some infinite prime of $F$; then either $F_{P}^{*}=R$ (real field) or $F_{P}^{*}=C$ (complex field), and there is an embedding $\epsilon_{P}: F \rightarrow F_{P}^{*}$.

Now let $A$ be any central simple $F$-algebra, and for $P$ an infinite prime of $F$, put $A_{P}^{*}=F_{P}^{*} \otimes_{F} A$, a central simple $F_{P}^{*}$-algebra. Then $A_{P}^{*} \cong(S)_{t}$ for some skewfield $S$ with center $F_{P}^{*}$, and there are only the following possibilities:

(i) $F_{P}^{*}=\mathrm{C}, \quad S=\mathrm{C}$.

(ii) $F_{P}^{*}=R, \quad S=R$.

(iii) $F_{P}^{*}=R, \quad S=H$ (= quaternion skewfield over $R$ ). In case (iii), we say that $P$ ramifies in $A$.

Hence for any prime $P$ (finite or infinite) of $F$, we say that $P$ ramifies in $A$ if and only if $A_{P}^{*}$ is not a full matrix algebra over $F_{P}^{*}$. The following theorems are rather deep.

(7.18). Theorem (HASse). If $A$ is not a full matrix algebra over $F$, then at least two primes (finite or infinite) of $F$ must ramify in $A$.

(7.19). Theorem (HASSE). $A$ nonzero element $\alpha \in F$ is the reduced norm $N(a)$ of some unit $a \in A$ if and only if $\epsilon_{P}(\alpha)>0$ at every infinite prime $P$ of $F$ which ramifies in $A$. Hence the group $I^{\prime}\{R\}$ defined in (7.17) is given by

$I^{\prime}\{R\}=\left\{\alpha R: \alpha \in F, \epsilon_{P}(\alpha)>0\right.$ at every infinite prime $P$ of $F$ ramified in $A\}$.

Call $A$ a totally definite quaternion algebra if $A$ is a skewfield with center $F$, such that for each infinite prime $P$ of $F, A_{P}^{*} \cong H$. This can occur only when $(A: F)=4$ and $F_{P}^{*}=R$ for every infinite prime $P$ of $F$.

(7.20). Theorem (EIChler). Suppose that $A$ is not a totally definite quaternion algebra, and let $J$ be a normal ideal in $A$. Then $J$ is a principal ideal if and only if the reduced norm $N(J)$ lies in $I^{\prime}\{R\}$.

8. Further results on genera. Throughout this section let $R$ be a Dedekind domain whose quotient field $F$ is an algebraic number field, 
and let $A$ be a semisimple $F$-algebra:

$$
\begin{aligned}
& A=A_{1} \oplus \cdots \oplus A_{r} \quad \text { (simple components). } \\
& A_{i} \cong\left(D_{i}\right)_{n_{i}}, \quad D_{i}=\text { skewfield with center } F_{i} . \\
& R_{i}=\text { integral closure of } R \text { in } F_{i} .
\end{aligned}
$$

Denote by $e_{i}$ the central idempotent of $A$ generating $A_{i}$. As in $\$ 7$, let $I\left\{R_{k}\right\}$ be the ideal group in $F_{k}$. Let $N_{k}: A_{k} \rightarrow F_{k}$ be the reduced norm map, and as in (7.17) and (7.19), let

$$
I^{\prime}\left\{R_{k}\right\}=\left\{N_{k}(a) \cdot R_{k}: a=\text { unit in } A_{k}\right\} .
$$

Each central idempotent $e \in A$ is expressible as a sum $e_{i_{1}}+\cdots+e_{i_{i}}$, say. Define

$$
I\{e\}=\prod_{\nu=1}^{s} I\left(R_{i_{v}}\right), \quad I^{\prime}\{e\}=\prod_{\nu=1}^{s} I^{\prime}\left\{R_{i_{\nu}}\right\} .
$$

These will be used later.

Now let $M$ be a left $\Lambda$-lattice, where $\Lambda$ is any $R$-order in $A$, and set

$$
E(M)=\operatorname{Hom}_{\Lambda}(M, M), \quad E(F M)=\operatorname{Hom}_{A}(F M, F M) .
$$

(8.2a). Lemma. $E(M)$ is an $R$-order in $E(F M)$. If $\Lambda$ is a maximal order in $A$, then $E(M)$ is a maximal $R$-order in $E(F M)$.

We shall view $M$ as left $\Lambda$-, right $E(M)$-lattice. Define

$$
e_{M}=\sum_{e_{i} M \neq 0} e_{i}=e_{i_{1}}+\cdots+e_{i_{s}} \text { (say). }
$$

Then $e_{M}$ is a central idempotent of $A$ which depends only on $F M$, and we have

$$
E(F M) \cong \sum_{\nu=1}^{s} \cdot\left(D_{i_{v}}\right)_{k_{v}}
$$

where $A_{i_{v}} \cdot F M$ is a direct sum of $k_{\nu}$ irreducible $A$-modules. Now let $\delta(\Lambda)$ be the family of all left $\Lambda$-lattices $M$ such that no simple component of $E(F M)$ is a totally definite quaternion algebra (see end of $\S 7)$. Thus $M \in \mathcal{S}(\Lambda)$ if and only if for each $\nu, 1 \leqq \nu \leqq s$, either $k_{\nu}>1$ or else $D_{i_{\nu}}$ is not a totally definite quaternion algebra. We shall say that the $\Lambda$-lattice $M$ satisfies the Eichler condition if and only if $M \in \delta(\Lambda)$; of course this is really a condition on the $A$-module $F M$.

We have denoted by $\Gamma(M)$ the genus of $M$, and by $|\Gamma(M)|$ the number of $\Lambda$-isomorphism classes in $\Gamma(M)$. Suppose in particular that $\Lambda_{0}$ is a maximal $R$-order in $A$, and that $M_{0}$ is a left $\Lambda_{0}$-lattice. Since $E\left(M_{0}\right)$ is a maximal order in $E\left(F M_{0}\right)$, it can be written as 


$$
E\left(M_{0}\right)=\sum_{\nu=1}^{s} \cdot \Omega_{i_{p}}, \text { where } e_{M_{0}}=e_{i_{1}}+\cdots+e_{i_{s}},
$$

and $\Omega_{i v}$ is a maximal $R_{i_{\nu}}$-order in the $i_{\nu}$ th simple component of $E\left(F M_{0}\right)$. Each full left $E\left(M_{0}\right)$-ideal $J$ in $E\left(F M_{0}\right)$ likewise decomposes as $J=\sum \cdot J_{i_{v}}$. Define the reduced norm

$$
N^{*}(J)=\prod_{i=1}^{s} N_{i_{v}}\left(J_{i_{v}}\right) \in I\left\{e_{M_{0}}\right\}
$$

From $\S 7$ we see that if $J$ is principal then $N^{*}(J) \in I^{\prime}\left\{e_{M_{0}}\right\}$; and if $M_{0} \in \mathcal{S}\left(\Lambda_{0}\right)$, then the converse holds true by (7.20). Hence we obtain

(8.5). Theorem. Let $\Lambda_{0}$ be a maximal R-order in $A$, and let $M_{0}, N_{0}$ be lefı $\Lambda_{0}$-lattices.

(i) $N_{0} \in \Gamma\left(M_{0}\right)$ if and only if $F N_{0} \cong F M_{0}$.

(ii) Each $N_{0} \in \Gamma\left(M_{0}\right)$ is $\Lambda_{0}$-isomorphic to $M_{0} J$ for some full left $E\left(M_{0}\right)$-ideal $J$ in $E\left(F M_{0}\right)$.

-(iii) For $J_{1}, J_{2}$ full left $E\left(M_{0}\right)$-ideals in $E\left(F M_{0}\right), M_{0} J_{1} \cong M_{0} J_{2}$ if and only if $J_{1}=J_{2} a$ for some unit $a \in E\left(F M_{0}\right)$.

(iv) The reduced norm map $N^{*}$ maps the set of all full left $E\left(M_{0}\right)$ ideals onto $I\left\{e_{M_{0}}\right\}$, carrying principal ideals onto $I^{\prime}\left\{e_{M_{0}}\right\}$.

(v) If $M_{0}$ satisfies the Eichler condition, then $J$ is principal if and only if $N^{*}(J) \in I^{\prime}\left\{e_{M_{0}}\right\}$. Therefore

$$
\left|\Gamma\left(M_{0}\right)\right|=\left[I\left\{e_{M_{0}}\right\}: I^{\prime}\left\{e_{M_{0}}\right\}\right],
$$

and this index is finite.

References. Chevalley [41], Jacobinski [128], [129].

Consider now an arbitrary $R$-order $\Lambda$ in $A$, and choose some maximal order $\Lambda_{0}$ containing $\Lambda$. Let $f$ be a full two-sided $\Lambda_{0}$-ideal in $\Lambda$ (such exist: take $\mathfrak{f}=\alpha \Lambda_{0}$, with suitable $\alpha \in R$ ). Given a $\Lambda$-lattice $M$, we may form $\Lambda_{0} M$ in $F M$, and then $M_{0}=\Lambda_{0} M$ is a $\Lambda_{0}$-lattice in $F M$. Call a full left ideal $J$ in $E\left(M_{0}\right)$ relatively prime to f if for each maximal ideal $P$ of $R$, either $\mathfrak{f}_{P}=\left(\Lambda_{0}\right)_{P}$ or else $J_{P}=\left\{E\left(M_{0}\right)\right\}_{P}$.

(8.6). Theorem (JACOBINSKI [128]). Every $\Lambda$-lattice $N$ in the genus of $M$ is $\Lambda$-isomorphic to $M \cap M_{0} J$ for some full left ideal $J$ in $E\left(M_{0}\right)$ relatively prime to f. Further, $M \cap M_{0} J \cong M$ if and only if $J=E\left(M_{0}\right) a$ for some $a \in E(M)$.

Let $\mathfrak{a}=\prod \mathfrak{a}_{i}$ be an ideal in the ideal group $I\left\{e_{M}\right\}$; here $i$ ranges over all indices such that $e_{i} M \neq 0$. We call a relatively prime to $f$ if for each maximal ideal $P$ of $R$ such that $\mathfrak{f}_{P} \neq\left(\Lambda_{0}\right)_{P}$, the numerator and denominator of each $R_{i}$-ideal $\mathfrak{a}_{i}$ in $F_{i}$ is prime to $P$. The collection 
of all such ideals $\mathfrak{a}$ is a subgroup $I\left\{e_{M}, \mathfrak{f}\right\}$ of finite index in $I\left\{e_{M}\right\}$. We now let $I^{\prime}\left\{e_{M}, \mathfrak{f}\right\}$ be the subgroup of $I\left\{e_{M}, \mathfrak{f}\right\}$ generated by all principal ideals of the form $N^{*}\left(\Lambda_{0} a\right), a \in E(M)$, which are prime to $\mathfrak{f}$. Define

$$
V(M, \mathfrak{f})=I\left\{e_{M}, \mathfrak{f}\right\} / I^{\prime}\left\{e_{M}, \mathfrak{f}\right\},
$$

a finite multiplicative group.

(8.8). Theorem (Jacobinski [128], [129]). The mapping which assigns to $M \cap M_{0} J$ the coset of $N^{*}(J)$ in $V(M, \mathfrak{f})$ carries the set of $\Lambda$-isomorphism classes in $\Gamma(M)$ onto $V(M, \mathfrak{f})$. When $M$ satisfies the Eichler condition, the mapping is one-to-one, and

$$
|\Gamma(M)|=\text { order of } V(M, \mathfrak{f}) \text {. }
$$

This result has many interesting consequences:

(8.9). Corollary (JAcobinski [128], [129], RoĬter [212]). Given an $R$-order $\Lambda$, there exists a positive integer $k$ depending only on $\Lambda$, such that for any pair of $\Lambda$-lattices $M$ and $N$, we have $N \in \Gamma(M)$ if and only if $M^{(k)} \cong N^{(k)}$.

(8.10). CoRollary (JACOBInski [128]). For each given $R$-order $\Lambda$, there exists a finite extension field $F^{\prime}$ of $F$ such that for any pair of $\Lambda$-lattices $M$ and $N, N \in \Gamma(M)$ if and only if

$$
R^{\prime} \otimes_{R} M \cong R^{\prime} \otimes_{R} N \quad \text { as } R^{\prime} \otimes_{R} \Lambda \text {-lattices. }
$$

Here, $R^{\prime}$ denotes the integral closure of $R$ in $F^{\prime}$.

We remark that, in general, $R^{\prime} \otimes M \cong R^{\prime} \otimes N$ need not imply that $M \cong N$ (see Berman-Gudivok [27], for instance).

The strict genus of the $\Lambda$-lattice $M$, denoted by $\Gamma^{s}(M)$, consists of all $N \in \Gamma(M)$ such that $\Lambda_{0} M \cong \Lambda_{0} N$ for some maximal order $\Lambda_{0}$ containing $\Lambda$. Jacobinski [128] showed that when $M$ satisfies the Eichler condition, then this isomorphism holds for every $\Lambda_{0}$ as soon as it is valid for one $\Lambda_{\mathbf{0}}$. Hereafter let $M$ satisfy the Eichler condition; up to $\Lambda$-isomorphism, the $\Lambda$-lattices in $\Gamma(M)$ are of the form $N=M \cap M_{0} J$ as in (8.6), and it turns out that $N \in \Gamma^{s}(M)$ if and only if $J=E\left(M_{0}\right) b$ for some $b \in E\left(M_{0}\right)$. Using the fact that each class of full left ideals in $E\left(M_{0}\right)$ contains an ideal relatively prime to $\mathfrak{f}$, one obtains

(8.11). Theorem (Jacobinski [128]). If $M$ satisfies the Eichler condition, the genus $\Gamma(M)$ splits into $h$ strict genera, with h equal to the number of classes of full left ideals in $E\left(M_{0}\right)$. Each strict genus $\Gamma^{8}(M)$ contains the same number $\left|\Gamma^{s}(M)\right|$ of $\Lambda$-isomorphism classes. Further, $h=\left[I\left\{e_{M}\right\}: I^{\prime}\left\{e_{M}\right\}\right]$. 
Takahashi [238] and Jacobinski [128] compute $\left|\Gamma^{s}(M)\right|$ as the number of double cosets in some group.

(8.12). Theorem (JAcobinski [128], [129]). Let $M$ and $N$ be $\Lambda$-lattices satisfying the Eichler condition. Let $T$ be a faithful ${ }^{6} \Lambda_{0}$-lattice satisfying the Eichler condition, where $\Lambda_{0}$ is a maximal order containing $\Lambda$. Then $N \in \Gamma^{s}(M)$ if and only if $M+T \cong N+T$ as $\Lambda$-lattices.

(8.13). Theorem. Let $\Lambda$ be an order in a simple algebra, and let $M, N$ be $\Lambda$-lattices in the same genus satisfying the Eichler condition. Then $N$ is isomorphic to a maximal sublattice of $M$.

References. Drozd [70a], Jacobinski [130]. This provides a partial answer to a question raised by Roüter [212].

9. Projective modules and relative projective modules. Let $R$ be a Dedekind ring with quotient field $F$, and let $\Lambda$ be an $R$-order in the $F$-algebra $A$. A left $\Lambda$-lattice $M$ is projective if $M$ is a direct summand of a free module, or equivalently, if every exact sequence $0 \rightarrow U \rightarrow V$ $\rightarrow M \rightarrow 0$ of $\Lambda$-modules splits.

(9.1). THEorem. Let $R$ be a discrete valuation ring with maximal ideal $P$, and set $\bar{R}=R / P, \bar{\Lambda}=\Lambda / P \Lambda, \bar{M}=M / P M$.

(i) $A$ left $\Lambda$-lattice $M$ is projective if and only if $\bar{M}$ is a projective $\bar{\Lambda}$-module.

(ii) If $M$ and $N$ are projective $\Lambda$-modules, then $M \cong N$ if and only if $\bar{M} \cong \bar{N}$.

References. Nakayama [161]-[163], Reiner [181], Swan [232], CR $\$ 77$.

Much deeper results can be obtained when $\Lambda=R G$, as indicated below.

(9.2). Theorem. Let $R$ be a discrete valuation ring in the algebraic number field $F$, and let $G$ be a finite group. For a pair of projective $R G$ lattices $M, N$, we have $M \cong N$ if and only if $F M \cong F N$.

References. This was first proved by Swan [232]. Other proofs are due to Rim (unpublished), Giorgiutti [89], and Bass [8]; these make use of the nonsingularity of the Cartan matrix of $\bar{R} G$ (see CR $\$ 77$ ). A proof of an entirely different nature may be found in Hattori [107].

The preceding theorem is one of the key steps in proving the following striking result on projective $R G$-lattices in the global case.

(9.3). Theorem (Swan [232]). Let $R$ be any Dedekind domain of

${ }^{6}$ This means that for $x \in \Lambda_{0}, x T=0$ implies $x=0$. 
characteristic zero, with quotient field $F$, and let $G$ be a finite group of order $g$. Suppose that every prime divisor of $g$ is a nonunit in $R$. Let $P$ range over the maximal ideals of $R$.

(i) An RG-lattice $M$ is projective if and only if for each $P$ dividing $g R, M_{P}$ is $R_{P} G$-projective, or equivalently, $M / P M$ is $(R / P) G$-projective.

(ii) If $M$ is a projective $R G$-lattice, then $F M$ is $F G$-free, and for each $P, M_{P}$ is $R_{P} G$-free and $M / P M$ is $(R / P) G$-free. Hence an $R G$-lattice is projective if and only if it is in the same genus as some free $R G$-lattice.

(iii) For each projective RG-lattice $M$ there exists a decomposition

$$
M \cong R G^{(r)}+L
$$

for some $r \geqq 0$ and some projective left ideal $L$ in $R G$. Here, $R G^{(r)}$ denotes the direct sum of $r$ copies of $R G$. The ideal $L$ is in the same genus as $R G$, and $F \cdot L=F G$.

Remarks. 1. Part (i) is valid more generally: if $\Lambda$ is an $R$-order in a separable algebra over $F$, then a $\Lambda$-lattice $M$ is projective if and only if $M_{P}$ is projective for each $P$ dividing the Higman ideal $i(\Lambda)$.

2. The crucial result in the above is part (ii), and especially the fact that if $M$ is projective then $F M$ is free. This readily implies the remaining results (see $\mathrm{CR} \$ 78$ ). It also shows that the group ring $R G$ contains no nontrivial idempotent elements; for a direct proof of this, see Coleman [44].

Now let $M, N$ be any $R G$-lattices; then $M \otimes_{R} N$ is also an $R G$ lattice, with the action of $G$ given by

$$
x(m \otimes n)=x m \otimes x n, \quad x \in G, m \in M, n \in N .
$$

(9.4). TheOREM (SWAN [232]). If $M$ is any $R$-free $R G$-lattice, then the $R G$-lattice $R G \otimes_{R} M$ (with diagonal action of $G$, as above) is $R G$-free on (FM:F) generators. Consequently if $L$ is any $R G$-lattice and $N$ is $R G$-projective, then $L \otimes_{R} N$ is also $R G$-projective.

We turn now to a discussion of the relations between $R G$-lattices and $R H$-lattices, where $H$ is a subgroup of $G$. Given an $R H$-lattice $V$, define the induced $R G$-lattice $V^{G}$ as

$$
V^{G}=R G \otimes_{R H} V,
$$

with the elements of $G$ acting on the left on the first factor only. If $G=U x_{i} H$, then $V^{G}=\sum x_{i} \otimes V$ (as $R$-modules). On the other hand, each $R G$-lattice $M$ can be restricted to $H$ to give an $R H$-lattice $M_{H}$.

(9.5). Theorem. Let $H \subset K \subset G$ be finite groups, $V$ an $R H$-lattice, $M$ an $R G$-lattice, and let $V^{\prime}$ denote the contragredient of $V$ (see end of $\S 3$ ). 
Then:

(i) Induction is transitive: $\left(V^{K}\right)^{G} \cong V^{G}$.

(ii) Frobenius reciprocity theorem holds: $V^{G} \otimes_{R} M \cong\left(V \otimes_{R} M_{H}\right)^{G}$.

(iii) $\left(V^{\prime}\right)^{G} \cong\left(V^{G}\right)^{\prime}$.

(iv) The Mackey subgroup theorem and tensor product theorem are valid.

References. The proofs in $\mathrm{CR} \$ \$ 38,43,44$ carry over unchanged to the present situation. The Mackey theorems (Mackey [153]) are stated in CR(44.2), (44.3).

If $M, N$ are lattices we write $M \mid N$ to indicate that $M$ is isomorphic to a direct summand of $N$.

(9.6). Theorem. Let $H$ be a subgroup of $G, V=R H$-lattice, $M=R G$ lattice.

(i) $V \mid\left(V^{G}\right)_{H}$ always.

(ii) $M \mid\left(M_{H}\right)^{G}$ if $[G: H]$ is a unit in $R$.

References. See CR(63.6), (63.7); the second result is originally due to D. G. Higman [116].

We introduce next the concept of relative projective lattices. Let $M$ be an $R G$-lattice, and $H$ a subgroup of $G$. We call $M(G, H)$-projective if any one of the following equivalent conditions holds true:

(i) $M \mid\left(M_{H}\right)^{G}$.

(ii) $M \mid L^{G}$ for some $R H$-lattice $L$.

(iii) Every exact sequence of $R G$-lattices $0 \rightarrow X \rightarrow Y \rightarrow M \rightarrow 0$ which is $R H$-split is also $R G$-split.

(iv) Every exact sequence of $R G$-lattices $0 \rightarrow M \rightarrow Y \rightarrow W \rightarrow 0$ which is $R H$-split is also $R G$-split.

(v) Let $G=\bigcup x_{i} H$. Then there exists an element $\gamma \in \operatorname{Hom}_{R H}(M, M)$ such that $\sum_{i} x_{i} \gamma x_{i}^{-1}=$ identity map on $M$. (See CR $\S 63$.)

This concept plays a vital role in Green's theory of vertices and sources of indecomposable $R G$-lattices. While the theory originally dealt with $F G$-modules, the entire discussion carries over almost unchanged to $R G$-lattices, provided the domain $R$ is sufficiently nice so that the Krull-Schmidt theorem holds for $R G$-lattices (see 5.2).

(9.7). THEOREM. Let $R$ be a complete discrete valuation ring, and let $M$ be an indecomposable RG-lattice.

(i) There exists a subgroup $H$ of $G$, called the vertex of $M$, such that $M$ is $(G, H)$-projective, and such that for $K$ a subgroup of $G, M$ is $(G, K)$-projective if and only if $K$ contains a conjugate of $H$. The subgroup $H$ is uniquely determined by $M$ up to conjugacy in $G$.

(ii) If $H=$ vertex of $M$, then there exists an indecomposable $R H$-lattice 
$L$ (unique up to conjugacy) such that $M \mid L^{G}$. Call $L$ the source of $M$.

(iii) If the rational prime $p$ is a nonunit in $R$, then the vertex of an indecomposable lattice is always a p-group.

(iv) Let $K$ be any subgroup of $G$ such that $[G: K]$ is a power of $p$, where $p$ is a prime which is a nonunit in $R$. Let $V$ be any absolutely indecomposable $R G$-lattice. Then $V^{G}$ is an absolutely indecomposable $R G$-lattice.

References. Green [90], [91], [93], CR §65.

Other references for this section: Thompson [253], Dress [59], [61]-[63], Conlon [44a]-[44d].

10. Grothendieck groups and Whitehead groups. Let $\Lambda$ be a ring with unity element, and let $\mathfrak{C}$ be some category of $\Lambda$-modules (always left finitely generated modules). Let $\delta$ be some collection of short exact sequences

$$
0 \rightarrow L \rightarrow M \rightarrow N \rightarrow 0, \quad L, M, N \in \mathfrak{C} .
$$

Form the free abelian group $\mathfrak{A}$ on the symbols $(M)$, one for each isomorphism class of $\Lambda$-modules in $\mathcal{C}$. Let $\mathfrak{A}_{0}$ be the subgroup of $\mathfrak{A}$ generated by all expressions $(M)-(L)-(N)$, where in (10.1) we use all sequences in the given collection $\delta$. We call the additive group $\mathfrak{A} / \mathfrak{A}_{0}$ the Grothendieck group of $\mathfrak{C}$ relative to $\mathcal{S}$, and denote it by $K(\mathcal{e}, \mathcal{s})$. For each $M \in \mathfrak{C}$ the image of $(M)$ in $K(\mathbb{e}, s)$ will be written as $[M]$.

As general references for this topic, we cite Bass [14], Heller [109], Swan [235].

Of special interest for us are the following cases:

(10.2). Choose $\mathcal{C}$ the category of all $\Lambda$-modules, $\varsigma$ the collection of all short exact sequences from $\mathrm{C}$. In this case $K(\mathrm{e}, \delta)$ is called the Grothendieck group of $\Lambda$, and will be denoted by $K(\Lambda)$ for brevity.

(10.3). Take $\mathcal{e}$ to be the category of all projective $\Lambda$-modules, and $\mathcal{S}$ the set of all short exact sequences from $\mathcal{C}$ (these are necessarily split). We call this $K(\mathcal{C}, \mathrm{s})$ the projective class group of $\Lambda$, and denote it by $P(\Lambda)$. (See Rim [200], [201].)

For the remainder of this section, we assume that $\Lambda$ is an $R$-order in the $F$-algebra $A$, where $R$ is any noetherian domain.

(10.4). Let $\mathcal{C}$ be the category of all $\Lambda$-lattices, $S$ the set of all short exact sequences from $\mathfrak{C}$. Denote by $K_{f}(\Lambda)$ the Grothendieck group $K(\mathcal{S}, \mathcal{e})$ thus obtained. An easy argument (see Swan [232]) shows that the obvious map $[M] \rightarrow[M]$ gives an isomorphism $K_{f}(\Lambda) \cong K(\Lambda)$.

${ }^{7}$ See (5.4). 
The Grothendieck group $K(A)$ is easily described: if $X_{1}, \cdots, X_{n}$ are a full set of nonisomorphic irreducible $A$-modules, then $K(A)$ is the free abelian group with basis $\left[X_{1}\right], \cdots,\left[X_{n}\right]$. (The same holds true for any artinian ring $A$.) Thus when $G$ is a finite group and char $F=0$, we may identify $K(F G)$ with the ring of generalized characters of $F G$-modules. On the other hand when char $F \neq 0$, we may identify $K(F G)$ with the ring of generalized Brauer characters of $F G$-modules (see CR $\S \S 38,82$ ).

In analyzing the structure of $K(\Lambda)$, one begins by comparing $K(\Lambda)$ with $K\left(\Lambda_{S}\right)$, where $\Lambda_{S}$ is gotten from $\Lambda$ by passing from $R$ to a ring of quotients $R_{S}$. Specifically, we quote

(10.5). Theorem (Swan [232], [234]). Let $S$ be any multiplicative subset of $R$ (with $1 \in S, 0 \notin S$ ), and let $R_{S}$ be the ring of quotients $\{\alpha / \beta: \alpha \in R, \beta \in S\}$. Set $\Lambda_{S}=R_{S} \otimes_{R} \Lambda$. Then there is an exact sequence of additive groups.

$$
\sum_{P}^{\cdot} K\left(\frac{\Lambda}{P \Lambda}\right) \stackrel{\eta}{\rightarrow} K(\Lambda) \stackrel{\theta}{\rightarrow} K\left(\Lambda_{S}\right) \rightarrow 0,
$$

where in the first direct sum $P$ ranges over all those prime ideals in $R$ for which $P \cap S$ is nonempty. The map $\theta$ is given by $[M] \rightarrow\left[M_{S}\right]$, $M=\Lambda$-module. The map $\eta$ is defined on each $K(\Lambda / P \Lambda)$ by vierwing $(\Lambda / P \Lambda)$-modules as $\Lambda$-modules.

The result can be greatly strengthened when $\Lambda$ is a group ring. Call a Dedekind domain $R$ semilocal if $R$ has only a finite number of prime ideals. The following difficult and important result is due to Swan [234]:

(10.6). Theorem. Let $R$ be a semilocal Dedekind domain, and let $G$ be a finite group. Then there is an isomorphism $\theta: K(R G) \cong K(F G)$, where $\theta$ is the map defined by $[M] \rightarrow[F M]$ (as in (10.5), with $S$ chosen to be the set of nonzero elements of $R$ ).

(10.7). Corollary. Let $G$ be a finite group, and let 3 be some finite set of prime ideals of the Dedekind domain $R$. There is an exact sequence of additive groups

$$
\sum_{P \notin \mathcal{J}}^{\cdot} K\left(\frac{R}{P} G\right) \stackrel{\eta}{\rightarrow} K(R G) \stackrel{\theta}{\rightarrow} K(F G) \rightarrow 0,
$$

where in the direct sum $P$ ranges over all nonzero prime ideals of $R$ dis-

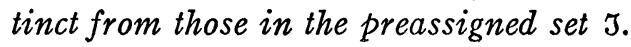


A projective $\Lambda$-module $M$ is special if $F M$ is free as $A$-module. Let $P_{0}(\Lambda)$ be the Grothendieck group of the category of all special projective $\Lambda$-modules, using for $S$ the collection of all sequences of such modules. Equivalently, $P_{0}(\Lambda)$ is the subgroup of $P(\Lambda)$ generated by special projectives. Denote by $C_{0}(\Lambda)$ the subgroup of $P_{0}(\Lambda)$ generated by the set of all differences

$\{[M]-[N], \quad M, N$ special projective $\Lambda$-modules, $F M \cong F N\}$.

Terminology:

$P(\Lambda)=$ projective class group of $\Lambda$.

$P_{0}(\Lambda)=$ special projective class group of $\Lambda$.

$C_{0}(\Lambda)=$ reduced special projective class group of $\Lambda$.

(See Rim [200], [201], Strooker [229], for further discussion of these concepts.)

There is an obvious mapping

$$
\alpha: P(\Lambda) \rightarrow K(\Lambda)
$$

gotten by mapping $[M]$ onto $[M]$, for $M$ any projective $\Lambda$-module. This induces a map $C_{0}(\Lambda) \rightarrow K(\Lambda)$.

An immediate consequence of (10.7) is

(10.8). Theorem (Swan [234]). Let $R$ be a Dedekind domain such that char $F \nmid[G: 1]$. Then there is an exact sequence of additive groups:

$$
C_{0}(R G) \stackrel{\alpha}{\rightarrow} K(R G) \stackrel{\theta}{\rightarrow} K(F G) \rightarrow 0 .
$$

When $R=$ alg. int. $\{F\}$, it follows at once from (9.3) that every projective $R G$-module is special, and is expressible as the direct sum of a free module and a projective left ideal $L$ in the group ring $R G$. Each element of $C_{0}(R G)$ can thus be expressed as a difference $[R G]-[L]$, with $L$ a projective left ideal in $R G$. The Jordan-Zassenhaus theorem (2.6) then implies that the group $C_{0}(R G)$ is finite, whence also $\operatorname{ker} \theta$ is a finite abelian group.

Keeping the assumption that $R=$ alg. int. $\{F\}$, let $\Lambda_{0}$ be any maximal $R$-order in $F G$ containing $R G$; then $[G: 1] \Lambda_{0} \subset R G$. By $(7.6)$ every left $\Lambda_{0}$-lattice is $\Lambda_{0}$-projective, whence

$$
K\left(\Lambda_{0}\right) \cong K_{f}\left(\Lambda_{0}\right) \cong P\left(\Lambda_{0}\right) .
$$

Since each $\Lambda_{0}$-module may be viewed as an $R G$-module by restriction of the operator domain, there is a homomorphism

$$
K\left(\Lambda_{0}\right) \stackrel{\text { res }}{\longrightarrow} K(R G) .
$$


In the other direction, we may define a map $\beta: C_{0}(R G) \rightarrow C_{0}\left(\Lambda_{0}\right)$ by

$$
\beta\{[M]-[N]\}=\left[\Lambda_{0} \otimes_{R G} M\right]-\left[\Lambda_{0} \otimes_{R G} N\right],
$$

where $M, N$ are special projective $R G$-modules with $F M \cong F N$. Using Theorem 10.6 and its corollaries, Swan [234] proved

(10.9). Theorem. Let $R=$ alg. int. $\{F\}$, and let $\Lambda_{0}$ be a maximal $R$-order in FG containing $R G$. Then there is a commutative diagram of additive groups, with exact rows:

$$
\begin{aligned}
& C_{0}(R G) \stackrel{\alpha}{\rightarrow} K(R G) \stackrel{\theta}{\rightarrow} K(F G) \rightarrow 0 \\
& \begin{array}{c}
\beta \downarrow \\
0 \rightarrow C_{0}\left(\Lambda_{0}\right) \stackrel{\alpha^{\prime}}{\rightarrow} \underset{\text { res }}{\rightarrow} K\left(\Lambda_{0}\right) \stackrel{\uparrow}{\theta^{\prime}} \stackrel{\uparrow}{\rightarrow} K(F G) \rightarrow 0
\end{array}
\end{aligned}
$$

Furthermore, both $\beta$ and res are epimorphisms, and $\operatorname{ker} \theta$ is the image of $C_{0}\left(\Lambda_{0}\right)$ in $K(R G)$.

REMARK. Swan shows by example that neither $\beta$ nor res o $\alpha^{\prime}$ need be monic.

Swan's Theorem 10.6 is the starting point for the explicit calculation of the additive structure of $K(R G), R=$ alg. int. $\{F\}$, due to Heller-Reiner [114], [115]. This calculation uses the concept of the Whitehead group $K^{1}(\Lambda)$ of a ring $\Lambda$, which we proceed to define. (General references: Bass [12], [14], Heller-Reiner [114], Heller [110], Swan [235].)

Let $\Lambda$ be an arbitrary ring with unity, and consider only finitely generated $\Lambda$-modules. Now form the collection of all ordered pairs $(M, \mu)$ in which $M$ ranges over all projective $\Lambda$-modules, and for each $M, \mu$ ranges over all automorphisms of $M$. Let $\mathfrak{B}$ be the free abelian group generated by all such pairs. On the other hand, consider commutative diagrams of projective $\Lambda$-modules, with exact rows and with $\lambda, \mu, \nu$ automorphisms:

$$
\begin{aligned}
0 \rightarrow & \stackrel{f}{\rightarrow} M \stackrel{g}{\rightarrow} N \rightarrow 0 \\
& \lambda \downarrow \stackrel{\mu}{ }{ }^{\mu}{ }^{\nu} \downarrow \\
0 \rightarrow L & \stackrel{f}{\rightarrow} M \stackrel{g}{\rightarrow} N \rightarrow 0
\end{aligned}
$$

Let $\mathfrak{B}_{0}$ be the subgroup of $\mathfrak{B}$ generated by all expressions

$$
(M, \mu)-(L, \lambda)-(N, \nu)
$$


arising from diagrams (10.10). Next, let $\mathfrak{B}_{1}$ be the subgroup of $\mathfrak{B}$ generated by all expressions

$$
\left(M, \mu \mu^{\prime}\right)-(M, \mu)-\left(M, \mu^{\prime}\right)
$$

where $M$ is any projective $\Lambda$-module, and $\mu, \mu^{\prime}$ are any automorphisms of $M$. Then we set

$$
K^{1}(\Lambda)=\mathfrak{B} /\left(\mathfrak{B}_{0}+\mathfrak{B}_{1}\right),
$$

an abelian additive group. The image of $(M, \mu)$ in $K^{1}(\Lambda)$ will be denoted by $[M, \mu]$.

(Alternatively, we may also define

$$
K^{1}(\Lambda)=\operatorname{inj} \lim \frac{G L(n, \Lambda)}{[G L(n, \Lambda), G L(n, \Lambda)]},
$$

with the obvious embedding of $G L(n, \Lambda)$ into $G L(n+1, \Lambda)$. See Bass [12], [14] for details.)

If $F^{\#}$ denotes the multiplicative group $F-\{0\}$, then $K^{1}(F) \cong F^{\#}$, the isomorphism being given by $[M, \mu] \rightarrow \operatorname{det} \mu$ for each vector space $M$ over $F$ and each automorphism $\mu$ of $M$. More generally, let $D$ be a skewfield, and let $D^{\#}$ be the commutator factor group of the multiplicative group $D-\{0\}$. Then $K^{1}(D) \cong D^{\sharp}$, where the isomorphism is given by mapping $[M, \mu]$ onto the Dieudonné determinant of $\mu$.

Now let $R$ be any noetherian domain, and let $\Lambda$ be an $R$-order in the $F$-algebra $A$. Denote by $K_{t}(\Lambda)$ the Grothendieck group of the category of $R$-torsion $\Lambda$-modules, using for $\delta$ the set of all exact sequences of such modules. For a pair of $\Lambda$-lattices $M, N$ satisfying $F M=F N$, define

$$
[M / / N]=[M /(M \cap N)]-[N /(M \cap N)] \in K_{t}(\Lambda) .
$$

Now let $\left[M^{*}, \mu^{*}\right] \in K^{1}(A)$, with $\mu^{*}$ any automorphism of the $A$-module $M^{*}$. Choose a $\Lambda$-lattice $M$ such that $F M=M^{*}$, and set

$$
\Delta\left[M^{*}, \mu^{*}\right]=\left[M / / \mu^{*}(M)\right] \in K_{t}(\Lambda) .
$$

(10.11). Theorem (Heller-Reiner [114]). The map $\Delta: K^{1}(A)$ $\rightarrow K_{t}(\Lambda)$ given above is well defined, and there is an exact sequence of additive groups

$$
K^{1}(A) \stackrel{\Delta}{\rightarrow} K_{t}(\Lambda) \stackrel{\eta}{\rightarrow} K(\Lambda) \stackrel{\theta}{\rightarrow} K(A) \rightarrow 0,
$$

with $\eta, \theta$ analogous to those in (10.5).

Next take $R=$ alg. int. $\{F\}$, and as in (10.9) let $\Lambda_{0}$ be a maximal 
$R$-order in $F G$ containing $R G$. Then there is a commutative diagram of additive groups with exact rows

$$
\begin{aligned}
& K^{1}(F G) \stackrel{\Delta^{\prime}}{\rightarrow} K_{t}\left(\Lambda_{0}\right) \stackrel{\eta^{\prime}}{\rightarrow} K\left(\Lambda_{0}\right) \stackrel{\theta^{\prime}}{\rightarrow} K(F G) \rightarrow 0 \\
& \begin{array}{cccc}
1 \downarrow & \gamma \downarrow & \gamma^{\prime} \downarrow & 1 \downarrow \\
K^{1}(F G) \stackrel{\Delta}{\rightarrow} K_{t}(R G) & \stackrel{\eta}{\rightarrow} K(R G) \stackrel{\theta}{\rightarrow} K(F G) \rightarrow 0
\end{array}
\end{aligned}
$$

in which $\gamma, \gamma^{\prime}$ are restriction maps. Since $\gamma^{\prime}$ is epic by (10.9), we obtain

$$
K(R G) \cong K(F G)+\operatorname{ker} \theta, \quad \operatorname{ker} \theta \cong \frac{K_{t}\left(\Lambda_{0}\right)}{\text { image of } \Delta^{\prime}+\operatorname{ker} \gamma} .
$$

We now give an explicit description of the finite abelian group ker $\theta$. From $\$ 7$ it follows easily that $\Delta^{\prime}$ is monic, and that

$$
K_{t}\left(\Lambda_{0}\right) \cong I\{1\}, \quad \text { image of } \Delta^{\prime} \cong I^{\prime}\{1\},
$$

using the notation of (8.1) and (8.2) with $A=F G$. For each $i, 1 \leqq i \leqq r$, choose a left $\Lambda_{0}$-lattice $M_{i}$ such that $F M_{i}$ is an irreducible $A_{i}$-module. We proceed to construct a set of "generalized decomposition numbers" corresponding to a fixed maximal ideal $P$ of $R$ dividing [G:1], as follows: let $\left\{P_{i 1}, \cdots, P_{i \lambda_{i}}\right\}$ be the maximal ideals of $R_{i}$ dividing $P$, where $1 \leqq i \leqq r$. Let $\bar{R}=R / P$, and let $Y_{1}, \cdots, Y_{w}$ be a full set of irreducible $\bar{R} G$-modules. For each $i$ and $j$, we may view $M_{i} / P_{i j} M_{i}$ as $\bar{R} G$-module; let $d_{i j}^{(k)}$ be the number of its composition factors which are isomorphic to $Y_{k}$, where $1 \leqq k \leqq w$. Now set

$$
u(P)=\left\{\prod_{i=1}^{r} \prod_{j=1}^{\lambda_{i}} P_{i j}^{a_{i j}} \in I\{1\}: \sum_{i, j} a_{i j} m_{i}^{-1} d_{i j}^{(k)}=0,1 \leqq k \leqq w\right\},
$$

where $m_{i}$ is the index of the skewfield $D_{i}$ occurring in (8.1). (Thus, $D_{i} \cong \operatorname{Hom}_{A}\left(F M_{i}, F M_{i}\right)$.)

(10.13). Theorem (Heller-Reiner [114], [115]). The kernel of $\gamma$ is precisely the subgroup $\prod_{P} u(P)$ of $I\{1\}$, where $P$ ranges over all maximal ideals of $R$ dividing [G:1]. Hence

$$
\begin{aligned}
& \operatorname{ker} \theta \cong I\{1\} /\left(I^{\prime}\{1\} \cdot \prod_{P} u(P)\right), \\
& K(F G) \cong \sum_{i=1}^{r} \cdot Z\left[F M_{i}\right],
\end{aligned}
$$

and $K(R G) \cong K(F G)+\operatorname{ker} \theta$ as additive groups.

It is also of interest to give an explicit formula for the reduced special projective class group $C_{0}(R G)$. 
(10.14). Theorem (Jacobinski [129]). Let $R=$ alg. int. $\{F\}$, and let $\mathfrak{f}$ be the product of all those maximal ideals $P$ of $R$ for which $R_{P} G$ is not a maximal order. Let $M$ be the left $R G$-lattice defined as follows: $M=R G^{(2)}$ if some simple component of $F G$ is a totally definite quaternion algebra, while $M=R G$ otherwise. Then

$$
C_{0}(R G) \cong V\{M, \mathfrak{f}\},
$$

where $V\{M, \mathfrak{f}\}$ is the group defined in (8.7).

REMARK. In Theorems 10.9, 10.13, 10.14 and 10.15 (below), instead of assuming that $R=$ alg. int. $\{F\}$, we need only assume that $R$ is a Dedekind domain whose quotient field is an algebraic number field, and that no prime divisor of $[G: 1]$ is a unit in $R$.

For $R$ any Dedekind domain, we may define a ring structure on $K(R G)$ by setting, for each pair of $R G$-lattices $M$ and $N$,

$$
[M] \cdot[N]=\left[M \otimes_{R} N\right] \text {. }
$$

Here, the elements of $G$ act diagonally on $M \otimes N$ (as in $\S 9$ ). If $1_{G}$ denotes the $R G$-lattice $R$ on which $G$ acts trivially, then $K(R G)$ is a commutative ring with unity element $\left[1_{G}\right]$. Despite Theorem 10.13 which gives such precise information about $K(R G)$ as additive group, very little is known about the multiplicative structure of $K(R G)$; see Obayashi [175], Stancl [227], Swan [234], Uchida [255]. The only general result which we quote is the striking theorem:

(10.15). Theorem (SwaN [234]). $(\operatorname{ker} \theta)^{2}=0$, where $\theta: K(R G)$ $\rightarrow K(F G)$ is given as in (10.9), and $R=$ alg. int. $\{F\}$.

Let $R$ be any Dedekind domain; as in $\$ 9$, we may define a restriction map $K(R G) \rightarrow K(R H),\left(x \rightarrow x_{H}\right)$, for $H$ any subgroup of $G$. Likewise the induction map $K(R H) \rightarrow K(R G),\left(y \rightarrow y^{G}\right)$, is well defined. The analogues of Theorem 9.5 , (i) and (ii), remain valid. If $\mathcal{H}$ is some collection of subgroups of $G$, set

$$
K_{\mathfrak{H C}}(R G)=\sum_{H \in \mathfrak{S C}}\{K(R H)\}^{G} \subset K(R G) .
$$

(10.16). TheOREM (SwAN [233]). Let $R$ be a Dedekind domain, and $m$ a positive integer such that $m \cdot K(F G) \subset K_{\mathfrak{H}}(F G)$. Then $m^{2} \cdot K(R G)$ $\subset K_{\mathfrak{H}}(R G)$. In particular, if $\mathfrak{H C}$ is the collection of all hyperelementary subgroups of $G$ (see CR $\S 42)$, then $K(R G)=K_{\mathfrak{H C}}(R G)$.

This circle of ideas involving the Frobenius reciprocity theorem has been systematically investigated by Lam [146]. 
Now let $R$ be a Dedekind domain of characteristic zero. There is a map

$$
\tau: P(R G) \rightarrow K(F G), \quad[M] \rightarrow[F M]
$$

and it is of interest to identify the image of $\tau$. The elements of $K(F G)$ can be thought of as generalized characters (CR $\S 38$ ), that is, as differences $\phi_{1}-\phi_{2}$ of characters $\phi_{1}, \phi_{2}$ afforded by $F G$-modules. Call an element $x \in G R$-singular if the order of $x$ is not a unit in $R$.

(10.17). THEOREM (SwAN [234]). Let $\chi$ be a generalized character, viewed as an element of $K(F G)$. Then $\chi$ lies in the image of $\tau$ if and only if $\chi(x)=0$ for every $R$-singular element $x \in G$.

Call $G$ p-solvable if each composition factor of $G$ is either a $p$-group or else has order prime to $p$.

(10.18). Theorem (Swan [234]). Let $R$ be a Dedekind domain of characteristic zero, and let $X$ be an FG-module whose character vanishes on all $R$-singular elements of $G$. Suppose that for each of those prime factors $p$ of $[G: 1]$ which are not units in $R$, the group $G$ is p-solvable. Then there exists a projective $R G$-lattice $M$ for which $X \cong F M$.

The result is no longer true if the hypothesis on $G$ is omitted.

The Whitehead group $K^{1}(R G)$ is considerably harder to deal with than $K^{0}(R G)$. Indeed, even the calculation of $K^{1}(R)$ is already difficult. Bass [13] has shown how to compute the $Z$-rank of $K^{1}(Z G)$. A complete discussion of these problems, as well as a wealth of material on related subjects, may be found in the reference by Bass [14].

Other references for this section: Krugljak [145], Roggenkamp [204], [207].

11. Commutative orders and related results. Throughout this section, $R$ is a Dedekind domain and $\Lambda$ is an $R$-order in the $F$-algebra $A$. The semiexpository paper by Faddeev [80] will serve as general reference for the material in this section.

For $J$ a full $R$-lattice in $A$, define $O_{l}(J), O_{r}(J)$ and $J^{-1}$ as at the beginning of $\$ 7$. Call $J$ right invertible if $J \cdot J^{-1}=O_{l}(J)$, or equivalently, if $J N=O_{l}(J)$ for some full $R$-lattice $N$ in $A$. If $J$ is right invertible, then $J^{-1}$ is left invertible, and $O_{r}\left(J^{-1}\right)=O_{l}(J)$.

Suppose $M$ is any faithful left $\Lambda$-lattice not necessarily contained in $A$. (Call $M$ faithful if for $\lambda \in \Lambda, \lambda M=0$ implies that $\lambda=0$.) Since $A$ acts on $F M$, we may define

$$
O_{l}(M)=\{x \in A: x M \subset M\}
$$


This is an $R$-order in $A$, called the left multiplier ring of $M$, and $O_{l}(M) \supset \Lambda$. We say that $M$ is an exact $\Lambda$-lattice if $\Lambda=O_{l}(M)$.

(11.1). Proposition. For $J$ a full left $\Lambda$-lattice in $A$, set

$$
J^{\prime}=\{x \in A: J x \subset \Lambda\} \cong \operatorname{Hom}_{\Lambda}(J, \Lambda) .
$$

Then $J$ is $\Lambda$-projective if and only if $J^{\prime} \cdot J=O_{r}(J)$.

(11.2). Proposition. Let $A$ be commutative, and suppose that $J$ is an exact full left $\Lambda$-lattice in $A$. Then the following statements are equivalent:

(i) $J$ is $\Lambda$-projective.

(ii) $J$ is invertible.

(iii) For each maximal ideal $P$ of $R$, the localization $J_{P}$ is a principal $\Lambda_{P}$-ideal in $A$.

Reference. Faddeev [80, Propositions 18.2 and 27.1].

Following Borevič-Faddeev [34], we introduce the concept of orders of cyclic index. Let $A$ be a commutative separable algebra over $F$, so $A$ is a direct sum of fields $F_{i}$, each of which is a finite separable extension of $F$. Let $R_{i}$ be the integral closure of $R$ in $F_{i}$. Then $\Lambda_{0}=\sum \cdot R_{i}$ is the unique maximal $R$-order in $A$. An arbitrary $R$-order $\Lambda$ is said to have cyclic index (in $\Lambda_{0}$ ) if $\Lambda_{0}=\Lambda+\Lambda \omega$ for some $\omega \in \Lambda_{0}$. Thus, orders of cyclic index are "relatively close" to being maximal orders.

(11.3). TheOREm (Borevič-FAdDeEv [34]). Let A be a commutative separable $F$-algebra, and $\Lambda$ an R-order in $A$ of cyclic index. Then every full left $\Lambda$-lattice in $A$ is invertible. Further, each full left $\Lambda$-lattice $M$ in a free $A$-module $A^{(s)}$ uniquely determines an ascending chain of $R$-orders in $A$ :

$$
\Lambda \subset \Lambda_{1} \subset \cdots \subset \Lambda_{s},
$$

such that for each $i, 1 \leqq i \leqq s$, there exists an exact full left $\Lambda_{i}$-lattice $J_{i}$ in $A$, and

$$
M \cong J_{1}+\cdots+J_{s} \quad \text { as } \Lambda \text {-lattices. }
$$

The ideal class of the product $J_{1} \ldots J_{s}$ in $\Lambda_{s}$ is also uniquely determined by $M$. This chain of $R$-orders, and this ideal class, are the only invariants of $M$, and we may in fact choose $J_{i}=\Lambda_{i}, 1 \leqq i \leqq s-1$, in the above decomposition.

References. See also Brooks [38].

Using a completely different approach, Bass [10] obtained the following fruitful result. 
(11.4). BAss' TheOREM. Let $\Lambda$ be a noetherian (commutative) integral domain whose integral closure $\tilde{\Lambda}$ in the quotient field of $\Lambda$ is finitely generated as $\Lambda$-module. Then every finitely generated torsionfree $\Lambda$-module is isomorphic to an external direct sum of ideals of $\Lambda$ if and only if every ideal of $\Lambda$ can be generated by two or less elements.

REMARKs. Let $F$ be the quotient field of $\Lambda$; for $M$ a $\Lambda$-lattice, we call $(F M: F)$ the rank of $M$. Suppose that $\Lambda$ is a noetherian domain for which every $\Lambda$-lattice is a direct sum of $\Lambda$-lattices of rank at most $k$. Bass then shows that for each maximal ideal $m$ of $\Lambda$, every ideal of $\Lambda_{\mathfrak{m}}$ can be generated by at most $k+1$ elements. This in turn implies (by a result of I. S. Cohen) that each nonzero prime ideal of $\Lambda$ is maximal, and that each ideal of $\Lambda$ can be generated by $\operatorname{Max}(2, k+1)$ elements. Taking $k=1$, we obtain the theorem in one direction.

The proof in the other direction is more difficult. Assume that $\tilde{\Lambda}$ is finitely generated as $\Lambda$-module, and that each ideal of $\Lambda$ can be generated by two elements. The key step is the proof that under these hypotheses, every ideal of $\Lambda$ is $\Lambda^{\prime}$-projective for some uniquely determined domain $\Lambda^{\prime}$ containing $\Lambda$.

A related reference is Swan [237].

(11.5). Corollary. Let $x$ be an indeterminate over the quotient field $F$ of the Dedekind domain $R$. Given any finitely generated projective left $R[x]$-module $M$, there exists an $R$-lattice $M_{0}$ such that

$$
M \cong R[x] \underset{R}{\otimes} M_{0}
$$

In particular, when $R$ is a principal ideal domain, every finitely generated projective $R[x]$-module is free.

References. The result for the case where $R$ is a principal ideal domain was proved by Seshadri [225], and then extended by Bass [10] to Dedekind domains.

As pointed out by Borevič-Faddeev [35], Bass' Theorem yields a partial converse to (11.3).

(11.6). Proposition. Let $A$ be a finite separable field extension of $F$, and let $\Lambda$ be an $R$-order in $A$. If every left $\Lambda$-lattice is isomorphic to an external direct sum of ideals of $\Lambda$, then $\Lambda$ is an order of cyclic index.

Roiter [215] obtains a partial generalization of (11.4) to the noncommutative case:

(11.7). TheOREM. Let $A$ be a separable F-algebra, not necessarily commutative, and let $\Lambda$ be an $R$-order in $A$. If every left ideal of $\Lambda$ can be 
generated by two elements, then every left $\Lambda$-lattice is isomorphic to an external direct sum of left ideals of $\Lambda$.

In the proof of this theorem, Roiter uses his concept of "divisibility" of modules (see $\$ 12$ ). We shall return in $\$ 13$ to the question as to other sufficient conditions which guarantee that every indecomposable $\Lambda$-lattice is isomorphic to an ideal in $\Lambda$.

We turn next to some other aspects of orders in commutative algebras. Let $A$ be a commutative separable $F$-algebra, and let $\Lambda_{0}$ be the unique maximal $R$-order in $A$. Let $\Lambda$ be any $R$-order in $A$, and $M$ a full $\Lambda$-lattice in $A^{(n)}$. Define $M_{0}=\Lambda_{0} M$, and consider the order ideals ord $\left(M_{0} / M\right), \operatorname{ord}\left(\Lambda_{0} / \Lambda\right)$, defined as at the end of $\S 1$.

(11.8). THEOREM (FRÖHLICH [87]). The relation

$$
\operatorname{ord}\left(M_{0} / M\right) \supset\left\{\operatorname{ord}\left(\Lambda_{0} / \Lambda\right)\right\}^{n}
$$

is always true, and these ideals of $R$ are equal if and only if $M$ is $\Lambda$-projective. Further, $M$ is $\Lambda$-projective if and only if $M$ is an external direct sum $\sum M_{i}$ with each $M_{i}$ a $\Lambda$-lattice in the same genus as $\Lambda$.

We remark that this surprisingly simple criterion for projectivity does not extend readily to the noncommutative case (see Ballew [4], $[4 a])$.

Next let $A$ be a field, and let $J$ be a full $R$-lattice in $A$. Then each power $J^{n}$ is also a full $R$-lattice in $A$, and $O_{l}(J) \subset O_{l}\left(J^{n}\right)$, but these orders need not be equal. We have called $J$ invertible if $J \cdot L=O_{l}(J)$ for some full $R$-lattice $L$ in $A$; if $J$ is invertible, so is each $J^{n}$. However, it may well happen that $J^{n}$ is invertible though $J$ itself is not. The most striking result of this type is as follows:

(11.9). Theorem (Dade-Taussky-Zassenhaus [52 ], [53 ]). Let $\Lambda$ be a Z-order in an algebraic number field $A$, where $(A: Q)=d>1$. Then for every full $\Lambda$-lattice $J$ in $A, J^{d-1}$ and all higher powers of $J$ are invertible.

This is in fact a special case of their more general result:

(11.10). Theorem. Let $\Lambda$ be a noetherian integral domain with quotient field $A$, such that every nonzero prime ideal of $\Lambda$ is a maximal ideal of $\Lambda$.

(i) For each full left $\Lambda$-lattice $J$ in $A$, some power of $J$ is invertible.

(ii) If the integral closure of $\Lambda$ in $A$ is finitely generated as $\Lambda$-module, then there exists a positive integer $n$ such that $J^{n}$ is invertible for every $J$.

(iii) If $\Lambda$ is an $R$-order (where $R=$ Dedekind domain) and $(A: F)=d$, then $J^{d-1}$ is invertible for every full $\Lambda$-lattice $J$ in $A$. 
Further references on this topic: Dade [47], Dade-RobinsonTaussky-Ward [50], Singer [226a] Dade-Taussky [51a].

12. Divisibility of modules. This concept of Roiter's [210], [211], [213]-[216] has already shown its importance in several investigations. To begin with, let $\Lambda$ be any noetherian ring (with 1 ), and consider only finitely generated left $\Lambda$-modules. If $M, N$ are $\Lambda$-modules, denote by $M \cdot \operatorname{Hom}(M, N)$ the additive subgroup of $N$ generated by the set of elements

$$
\left\{f(m): f \in \operatorname{Hom}_{\Lambda}(M, N), m \in M\right\} .
$$

We shall say that $M$ covers $N$ (notation: $M \succ N$ ) if $M \cdot \operatorname{Hom}(M, N)$ $=N$. For example, $M$ covers every direct summand of each of its homomorphic images. (Roiter uses the term "divides" in place of "covers," but this conflicts with the standard notation $U \mid V$ indicating that $U$ is a direct summand of $V$.)

Let $M^{\prime}$ be a submodule of $M$. Call $M^{\prime}$ supercharacteristic if $M^{\prime} \cdot \operatorname{Hom}\left(M^{\prime}, M\right)=M^{\prime}$. For example, if $M$ and $N$ are arbitrary modules, then $M \cdot \operatorname{Hom}(M, N)$ is a supercharacteristic submodule of $N$.

As usual let $M^{(k)}$ denote the direct sum of $k$ copies of $M$. An easy argument shows that $M \succ N$ if and only if there is a $\Lambda$-exact sequence $M^{(k)} \rightarrow N \rightarrow 0$ for some $k$. In particular, $\Lambda \succ N$ for every $N$.

A $D$-submodule of a $\Lambda$-module $M$ is a supercharacteristic proper submodule $M^{\prime}$ covered by $M$, and such that for $M^{\prime \prime}$ a supercharacteristic submodule of $M, M^{\prime}+M^{\prime \prime}=M$ implies $M^{\prime \prime}=M$. The sum of all $D$-submodules of $M$ is again a $D$-submodule, the largest such in $M$, and is denoted by $D(M)$. Its importance stems from

(12.1). Theorem (RoǏTer [213, Theorem 2]). Let $R$ be a complete discrete valuation ring, $\Lambda$ an $R$-order in the separable F-algebra $A$, and let $M$ be a left $\Lambda$-lattice. Then $D(M)=0$ if and only if for each $k$, every exact sequence of $\Lambda$-lattices of the form

$$
0 \rightarrow M_{1} \rightarrow M^{(k)} \rightarrow M_{2} \rightarrow 0
$$

is split. In particular, $\Lambda$ is hereditary if and only if $D(\Lambda)=0$.

We return briefly to the general situation for the following basic idea. Let $\Lambda$ be any noetherian ring; a left $\Lambda$-module $M$ is said to have a normal decomposition if $M$ can be expressed as a direct sum of nonzero submodules: $M=M_{1} \oplus \cdots \oplus M_{r}, r>1$, such that $M_{i} \succ M_{j}$ for $1 \leqq i<j \leqq r$. If no such decomposition exists, call $M$ normally indecomposable.

(12.2). THEOREM. Let $\Lambda$ be as in (12.1), and let $M, N$ be left $\Lambda$-lattices 
such that $N \succ M$. If $N$ is normally indecomposable, or if $\operatorname{Hom}_{\Lambda}(N, N)$ is commutative, then every exact sequence $M \rightarrow N \rightarrow 0$ is $\Lambda$-split.

Let $B$ be an arbitrary finite dimensional algebra over a field $F$, and assume $B$ has a unity element. If in place of $\Lambda$-lattices we consider finitely generated $B$-modules, then the analogues of Theorems 12.1 and 12.2 remain valid. The latter theorem is a key step in Roitter's recent proof [216] of the Brauer-Thrall conjecture:

If there exist infinitely many nonisomorphic indecomposable B-modules, there cannot be a uniform upper bound on their dimensions over $F$.

13. Hereditary orders and related results. Throughout this section let $R$ denote a Dedekind domain, and let $A$ be a separable $F$-algebra. An $R$-order $\Lambda$ is hereditary if every left ideal of $\Lambda$ is a projective $\Lambda$ module. In our case, this is equivalent to the condition that every left $\Lambda$-lattice be projective.

The material in this section comes from the work of Brumer [39], Drozd-Kiričenko [71], Drozd-Kiričenko-Roŭter ${ }^{8}$ [73], and Harada [104]-[106]. One may also consult the lecture notes by RoggenkampDyson [208b].

(13.1). Theorem. Let $\Lambda$ be an $R$-order in $A$.

(i) If $\Lambda$ is hereditary, so is every bigger order.

(ii) $\Lambda$ is hereditary if and only if for each maximal ideal $p$ of $R$, the order $\Lambda_{p}$ is hereditary.

(iii) $A$ decomposition of $A$ into simple components gives rise to an analogous decomposition of every hereditary order in $A$.

(iv) If $A$ is a simple algebra with center $F^{\prime}$, then the center $R^{\prime}$ of a hereditary $R$-order $\Lambda$ in $A$ is a Dedekind domain with quotient field $F^{\prime}$.

(v) If $\Lambda$ is hereditary, every $\Lambda$-lattice is isomorphic to an external direct sum of full $\Lambda$-lattices in irreducible $A$-modules.

Parts (ii)-(iv) of the preceding theorem enable us to reduce the study of hereditary orders to the case where $A$ is a central simple algebra, and $R_{p}$ is a discrete valuation ring. The main results in this case are as follows (Brumer [39]):

(13.2). Let $D$ be a skewfield with center $F$, and let $A=\operatorname{Hom}_{D}(V, V)$ $\cong(D)_{n}$, where $V$ is a right vector space over $D$ of dimension $n$. Let $\Delta$ be a maximal $R_{p}$-order in $D$; then its Jacobson radical rad $\Delta$ is the unique maximal two-sided ideal of $\Delta$ (see (7.13)), and we define the skewfield $\Omega$ (over $R / p$ ) by

$$
\Delta / \operatorname{rad} \Delta \cong(\Omega)_{l} \quad \text { for some } l \text {. }
$$

\footnotetext{
${ }^{8}$ Abbreviated as D-K-R [73] hereafter.
} 
On the other hand, we have

$$
F_{p}^{*} \otimes_{F} A \cong(B)_{m} \quad \text { for some skewfield } B,
$$

and indeed $m=\ln$.

A $\Delta$-chain $\mathcal{E}$ of period $r$ is a strictly decreasing chain of full right $\Delta$-lattices in $V$ :

$$
\varepsilon: \cdots E_{-1} \supset E_{0} \supset E_{1} \supset \cdots \supset E_{r-1} \supset E_{r} \supset \cdots
$$

such that $E_{k+r}=E_{k} \cdot(\operatorname{rad} \Delta)$ for each $k$. Set

$$
\Lambda=\operatorname{Hom}_{\Delta}(\varepsilon, \varepsilon)=\left\{f \in A: f\left(E_{k}\right) \subset E_{k} \text { for all } k\right\} .
$$

Then $\Lambda$ is a hereditary $R_{p}$-order in $A$, and

$$
\operatorname{rad} \Lambda=\left\{f \in A: f\left(E_{k}\right) \subset E_{k+1} \text { for all } k\right\} .
$$

The quotient $\Lambda / \operatorname{rad} \Lambda$ is a direct sum of $r$ full matrix algebras over the skewfield $\Omega$, and for $0 \leqq k \leqq r-1$, the $k$ th simple component $(\Omega)_{n_{k}}$ is the ring of $(\Lambda / \mathrm{rad} \Lambda)$-endomorphisms of $E_{k} / E_{k+1}$. The sequence $\left(n_{0}, n_{1}, \cdots, n_{r-1}\right)$ is called the invariant of $\mathcal{E}$, and satisfies $n_{0}+\cdots+n_{r-1}=m$; the invariant is defined up to cyclic permutation.

Now let us define

$$
P_{k}=\left\{f \in \Lambda: f\left(E_{k}\right) \subset E_{k+1}\right\}, \quad \Gamma_{k}=\operatorname{Hom}_{\Delta}\left(E_{k}, E_{k}\right) .
$$

Both $P_{k}$ and $\Gamma_{k}$ depend only on $k(\bmod r)$. There are precisely $r$ distinct maximal two-sided ideals in $\Lambda$, namely $P_{1}, \cdots, P_{r}$. There are precisely $r$ distinct maximal $R_{p}$-orders in $A$ containing $\Lambda$, namely $\Gamma_{1}, \cdots, \Gamma_{r}$, and their intersection is $\Lambda$. Furthermore, $\varepsilon$ is the set of all left $\Lambda$-, right $\Delta$-lattices in $V$, and $(\operatorname{rad} \Lambda) E_{k}=E_{k+1}$ for all $k$.

Conversely, if $\Lambda$ is a hereditary $R_{p}$-order in $A$, the set of all $(\Lambda, \Delta)$ lattices in $V$ forms a $\Delta$-chain $\mathcal{E}$ of period $r$ (for some $r \leqq n$ ), and $\Lambda=\mathrm{Hom}_{\Delta}(\varepsilon, \varepsilon)$. There is a one-to-one inclusion-reversing correspondence between $R_{p}$-orders containing $\Lambda$, and subchains of $\varepsilon$.

Let $M$ be an indecomposable $\Lambda$-lattice, where $\Lambda$ is a hereditary $R_{p}$-order in $A$; then $M$ is isomorphic to a full $\Lambda$-lattice in $V$, and Hom $_{\Lambda}(M, M)$ is a hereditary $R_{p}$-order in $D$. If $D$ has a unique maximal $R_{p}$-order $\Delta$ (which is certainly true when $D=F$, and also when $R$ is a complete discrete valuation ring), then $\operatorname{Hom}_{\Lambda}(M, M)=\Delta$; further, under this assumption, the invariant $\left(n_{0}, \cdots, n_{r-1}\right)$ completely determines the structure of $\Lambda$. Specifically, if $\Lambda^{\prime}$ is another hereditary $R_{p}$-order in $A$, whose invariant is a cyclic permutation of $\left(n_{0}, \cdots, n_{r-1}\right)$, then $\Lambda^{\prime}=x \Lambda x^{-1}$ for some unit $x \in A$. Finally, when $\operatorname{Hom}_{\Lambda}(M, M)=\Delta$, we may display $\Lambda$ as the set of all those matrices in $(\Delta)_{n}$ of the form 


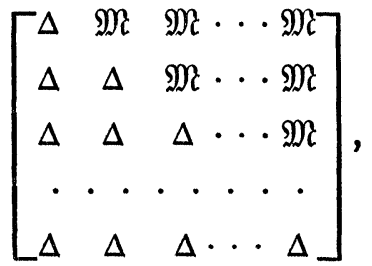

partitioned into $n_{i} \times n_{j}$ blocks all of whose entries belong to the indicated symbol (either $\Delta$ or $\mathfrak{M}$, where $\mathfrak{M}=\operatorname{rad} \Delta$ ).

Returning to the global case, we have

(13.3). Theorem. Let $A$ be a central simple F-algebra. Suppose that $\Lambda_{0}$ is some fixed $R$-order in $A$. Let there be given for each maximal ideal $p$ of $R$, a hereditary $R_{p}$-order $\Lambda(p)$ in $A$, satisfying $\Lambda(p)=\left(\Lambda_{0}\right)_{p}$ for all but a finite number of $p^{\prime} s$. Set

$$
\Lambda=\bigcap_{p} \Lambda(p) .
$$

Then $\Lambda$ is a hereditary $R$-order in $A$, and is the unique $R$-order such that $\Lambda_{p}=\Lambda(p)$ for all $p$. If we set

$$
M(p)=\Lambda \cap \operatorname{rad} \Lambda(p)
$$

then $M(p)$ is a full two-sided ideal in $\Lambda$, and satisfies

$$
(M(p))_{p}=\operatorname{rad} \Lambda(p), \quad(M(p))_{q}=\Lambda_{q} \quad \text { if } q \neq p .
$$

Let $\mathscr{S}(\Lambda)$ be the set of full two-sided $\Lambda$-ideals in $A$. An element $J \in \mathfrak{g}(\Lambda)$ is right $\Lambda$-invertible if there exists a $J^{\prime} \in \mathscr{g}(\Lambda)$ with $J J^{\prime}=\Lambda$.

(13.4). THEOREM. Let $\Lambda$ be a hereditary order in the central simple F-algebra $A$. Then $\mathfrak{g}(\Lambda)$ is the free abelian group on the generators $\{M(p): p=$ maximal ideal of $R\}$, and for $J \in \mathfrak{I}(\Lambda)$, we have $J^{-1}$ $=\{x \in A: x J \subset \Lambda\}$.

We may mention some other criteria for an order to be hereditary:

(13.5). THEOREM (D-K-R [73]). Let $\Lambda$ be an $R$-order in the separable algebra $A$. Then the following are equivalent:

(i) $\Lambda$ is hereditary.

(ii) There are no nonzero $D$-submodules of $\Lambda$ (see \$12).

(iii) Given any pair of $R$-orders $\Gamma_{1}, \Gamma_{2}$ in $A$ such that $\Lambda \subset \Gamma_{1} \subset \Gamma_{2}$, there exists an $R$-order $\Gamma_{3}$ in $A$ for which $\Lambda \subset \Gamma_{3}$ and $\Gamma_{1}=\Gamma_{2} \cap \Gamma_{3}$.

(iv) Every maximal $R$-order in $A$ containing $\Lambda$ is projective as left $\Lambda$-module.

For a hereditary order $\Lambda$ we know that every left $\Lambda$-lattice is iso- 
morphic to an external direct sum of left ideals ${ }^{9}$ in $\Lambda$. We have seen in $\$ 11$ that this property may hold for a larger class of orders. Here we shall sketch some further results in this direction.

(13.6). Let $\Lambda$ be an $R$-order in the separable $F$-algebra $A$. Call $\Lambda$ a Gorenstein order if any one of the following equivalent conditions is true:

(i) $\operatorname{Ext}_{\Lambda}^{2}(Y, \Lambda)=0$ for every left $\Lambda$-module $Y$.

(ii) $\operatorname{Ext}_{\Lambda}^{1}(M, \Lambda)=0$ for every left $\Lambda$-lattice $M$.

(iii) $\Lambda^{*}$ covers $\Lambda$ (see $\S 12$ ), where $\Lambda^{*}$ is the left $\Lambda$-module defined by $\Lambda^{*}=\operatorname{Hom}_{R}(\Lambda, R)$.

(iv) For every exact ${ }^{10}$ faithful left $\Lambda$-lattice $M, M \cdot \operatorname{Hom}_{\Lambda}(M, \Lambda)=\Lambda$.

(v) Every exact full left $\Lambda$-lattice in $A$ is right invertible."1

(Five more statements, each equivalent to (i), may be obtained by interchanging "left" and "right" in all of the above.)

References. D-K-R [73], Bass [11], Nazarova-Roìter [170].

A $\Lambda$-lattice $M$ is called weakly injective if every exact sequence $0 \rightarrow M \rightarrow X \rightarrow Y \rightarrow 0$ of $\Lambda$-lattices is split; in other words, $M$ is weakly injective if and only if $M$ is a $\Lambda$-direct summand of every bigger $\Lambda$-lattice of which $M$ is an $R$-direct summand.

(13.7). Proposition. Let $\Lambda$ be an $R_{p}^{*}$-order in a separable algebra. Then $\Lambda$ is a Gorenstein order if and only if every projective left $\Lambda$-lattice is weakly injective, or equivalently, if and only if every weakly injective $\Lambda$-lattice is projective.

(13.8). Definition. An $R$-order $\Lambda$ in $A$ is a Bass order if every $R$-order in $A$ containing $\Lambda$ is a Gorenstein order.

Every hereditary order is a Bass order. Roitter [215] proved that if every bigger $R$-order can be generated by two elements as left $\Lambda$-module, then $\Lambda$ is a Bass order. When $A$ is commutative, the converse also holds (Bass [11]).

(13.9). Theorem (D-K-R [73]). Let $\Lambda$ be an R-order in A. The following are equivalent:

(i) $\Lambda$ is a Bass order.

(ii) $\Lambda_{p}^{*}$ is a Bass order for each maximal ideal $p$ of $R$.

(iii) The set of all full two-sided $\Lambda$-lattices in $A$ forms a groupoid ${ }^{12}$ relative to proper $^{13}$ products.

${ }^{8}$ Indeed, part ( $v$ ) of Theorem 13.1 asserts an even stronger result.

${ }^{10}$ See definition immediately preceding (11.1).

"See beginning of $\$ 11$.

${ }^{12}$ See Jacobson [131, p. 132]. Compare this theorem with the earlier Theorem 7.10 .

${ }^{13}$ The product $J J^{\prime}$ is proper if replacing either factor by a larger lattice increases the product. 
(13.10). Definition. Let $V_{1}, \cdots, V_{t}$ be a full set of nonisomorphic irreducible $A$-modules, and let $M$ be a $\Lambda$-lattice. We shall say that $M$ has signature $\left(m_{1}, \cdots, m_{t}\right)$ if

$$
F M \cong V_{1}^{\left(m_{1}\right)}+\cdots+V_{t}^{\left(m_{t}\right)} \text {. }
$$

(13.11). Definition. Let $\Lambda$ be an $R_{p}^{*}$-order in the separable algebra $A$, and suppose that $\Lambda$ is a Bass order. We consider three types of orders:

Type I. The algebra $A$ has two simple components, and every indecomposable $\Lambda$-lattice has signature $(1,0),(0,1)$ or $(1,1)$ with the last type actually occurring.

Type II. The algebra $A$ is simple, and every indecomposable $\Lambda$ lattice has signature (1) or (2), with the latter type actually occurring.

Type III. The algebra $A$ is simple, and every indecomposable $\Lambda$-lattice has signature (1).

(13.12). Theorem (D-K-R [73, Theorem 9.7]). Every Bass $R_{p}^{*}$ order in a separable algebra is a ring direct sum of Bass orders of Types I, II and III.

Remarks. (i) Hereditary orders have Type III.

(ii) If $G$ is cyclic of order $p, Z_{p}^{*} G$ is of Type I.

(iii) Those $Z_{p}^{*}$-orders in the matrix ring $\left(Q_{p}^{*}\right)_{2}$, having finitely many indecomposable lattices, are of Type II (see Drozd-Kiričenko [71]).

(iv) Quadratic $Z_{p}^{*}$-rings are nonhereditary Bass orders of Type III (see Borevič-Faddeev [33]).

Detailed theorems on the structure of Bass orders are given in D-K-R [73]. Other references for this subject: Drozd-Roiter [74], Roggenkamp [204]. Also see Michler [160a], and references therein, for general results on hereditary orders.

14. Finiteness of the number of indecomposable representations. Let $R$ be a Dedekind domain with quotient field $F$, and let $\Lambda$ be an $R$-order in the separable $F$-algebra $A$. Denote by $n(\Lambda)$ the number of isomorphism classes of indecomposable left $\Lambda$-lattices. When is $n(\Lambda)$ finite?

(If the algebra $A$ is not semisimple, it follows from Faddeev [80, Proposition 25.1] that $n(\Lambda)$ is infinite. For this reason we limit our attention to separable algebras hereafter.)

The basic tool for passing from the global to the local case is as follows:

(14.1). Theorem. Let $F$ be an algebraic number field. Then $n(\Lambda)$ is finite if and only if for each maximal ideal $P$ of $R, n\left(\Lambda_{P}^{*}\right)$ is finite. 
References. Jones [135], Kneser [143], CR §81A.

Let $G$ be a finite group, $p$ a prime, and denote by $G_{p}$ a Sylow $p$ subgroup of $G$. The preceding theorem tells us that $n(R G)$ is finite if and only if $n\left(R_{P}^{*} G\right)$ is finite for each $P$.

(14.2). THEOREM. Let the rational prime $p$ be contained in the maximal ideal $P$ of $R$. Then $n\left(R_{P}^{*} G\right)$ is finite if and only if $n\left(R_{P}^{*} G_{p}\right)$ is finite.

REMARK. This follows readily from Theorem 9.6.

(14.3). THEOREM. Keep the above notation. If $G_{p}$ is not cyclic, or if $G_{p}$ is cyclic of order greater than $p^{2}$, then $n\left(R_{P}^{*} G_{p}\right)$ is infinite.

The above result was proved by Borevič-Faddeev [32, II] and Heller-Reiner [112, II]. It is also a special case of a more general result:

(14.4). THEOREM (DADE [48]). Let $R$ be any noetherian domain with quotient field $F$, and let $A$ be any finite dimensional $F$-algebra which is a direct sum of at least four subalgebras. Let $\Lambda$ be an $R$-order in $A$, and suppose there exists a maximal ideal $P$ of $R$ for which $\Lambda / P \Lambda$ is completely primary. ${ }^{14}$ Then $n(\Lambda)$ is infinite.

In particular, $n(\Lambda)$ is infinite whenever $\Lambda$ is an indecomposable $R_{P}^{*}$-order in a semisimple algebra having four or more simple components.

Generalizations of this result may be found in Drozd-Royter [74], Gudivok [95]-[101], Jacobinski [127].

In the other direction, we have

(14.5). THEOREM. Let $G_{p}$ be cyclic of order $p$ or $p^{2}$. Then $n\left(Z_{p}^{*} G_{p}\right)$ is finite.

References. Berman [25], Berman-Gudivok [29], Heller-Reiner $[112, \mathrm{I}]$.

(14.6). Corollary. The number of isomorphism classes of indecomposable ZG-lattices is finite if and only if for each prime $p$ dividing $[G: 1], G_{p}$ is cyclic of order $p$ or $p^{2}$.

In order to generalize (14.5) and (14.6), it is necessary to determine for which cyclic $p$-groups $H$ the number $n\left(R_{P}^{*} H\right)$ is finite. Many partial results have been obtained (see Gudivok [101] for a detailed description, as well as for calculations which give a solution in most cases). The complete solution was obtained by Jacobinski [127], who proved the following:

${ }^{14} \mathrm{~A}$ ring $\Gamma$ is completely primary if $\Gamma / \mathrm{rad} \Gamma$ is a skewfield. 
(14.7). THEOREM. Let $R=$ alg. int. $\{F\}$. For each maximal ideal $P$ of $R$, let $p$ be the rational prime contained in $P$, and let $e(P)$ be the largest integer for which $p R \subset P^{e(P)}$. Let $G_{p}$ be a Sylow p-subgroup of $G$. Then $n(R G)$ is finite if and only if for every maximal ideal $P$ dividing $[G: 1]$, one of the following conditions holds:

(i) $e(P)=1$ and $G_{p}$ is cyclic of order $p^{2}$.

(ii) $e(P) \leqq 2, p>3$, and $G_{p}$ is cyclic of order $p$.

(iii) $e(P) \leqq 3, p=3$, and $G_{p}$ is cyclic of order $p$.

As a matter of fact, Jacobinski [127] derived this as a consequence of his much more general theorem, which gives necessary and sufficient conditions for the finiteness of $n(\Lambda)$, where $\Lambda$ is any commutative $R_{P}^{*}$-order in a separable algebra. This problem has also been solved by Drozd-Roiter; their results are easier to state, though perhaps more difficult to apply.

(14.8). Theorem (Drozd-RoǏter [74]). Let $R=$ alg. int. $\{F\}$, and let $\Lambda$ be an $R$-order in the commutative semisimple algebra $A$. Let $\Lambda_{0}$ be the unique maximal $R$-order in $A$, and define $\operatorname{rad}\left(\Lambda_{0} / \Lambda\right)$ as the intersection of the maximal $\Lambda$-submodules of $\Lambda_{0} / \Lambda$. Then $n(\Lambda)$ is finite if and only if both of the following conditions are satisfied:

(i) As $\Lambda$-module, $\Lambda_{0} / \Lambda$ can be generated by two elements.

(ii) $\operatorname{rad}\left(\Lambda_{0} / \Lambda\right)$ is a cyclic $\Lambda$-module.

We mention one further general result.

(14.9). THEOREM (JACOBINSKI [129]). Let $F$ be an algebraic number field, and let $\Lambda$ be any $R$-order in the semisimple F-algebra $A$. Let $M$ be some fixed $\Lambda$-lattice, and let $B_{M}$ be the collection of direct summands of the lattices $M, M^{(2)}, M^{(3)}, \ldots$. Then the number of isomorphism classes of indecomposable lattices in $B_{M}$ is finite. In particular, there are finitely many isomorphism classes of indecomposable projective $\Lambda$-lattices.

Other references: Roggenkamp [208].

15. Representations of specific groups and orders. The aim of this section is to present a guide to the many articles containing explicit calculations and results on the classification of $\Lambda$-lattices, where $\Lambda$ is some order.

1. $Z G$-lattices, $G=$ cyclic group. $\$ 74$.

(a) $[G: 1]=p$. References: Diederichsen [57], Reiner [182], CR

(b) $[G: 1]=4$. References: Diederichsen [57], Knee [142], Matuljauskas [156], Roǐter [209], Troy [254]. 
(c) $[G: 1]=p^{2}$. References: Berman-Gudivok [27]-[29], HellerReiner [112], [113].

(d) $[G: 1]$ squarefree. References: Knee [142], Oppenheim [176].

2. $Z G$-lattices, $G$ arbitrary.

(a) $G=$ dihedral group of order $2 p$. References: Leahey [149], Lee [150], Matuljauskas [157], Nazarova-Roĭter [167].

(b) $G=$ nonabelian group of order $p q$ ( $p, q$ distinct primes). Reference: $\mathrm{Pu}$ [179].

3. In the following cases, there are infinitely many indecomposable $Z G$-lattices, and these are fully classified:

(a) $G=$ abelian $(2,2)$-group. Reference: Nazarova [164].

(b) $G=A_{4}$. Reference: Nazarova [165].

4. Further references on integral representations of groups: Barannik-Gudivok [6], Berman [21]-[25], Berman-Gudivok [27][29], Berman-Lihtman [30], Drobotenko [65], Gudivok [95]-[101], Gudivok-Rud'ko [102], Jones [136], [137], Kneser [143], Matuljauskas-Matuljauskene [159], Nazarova-Roŏter [168], [171], Reiner [187], [190], [191], Roggenkamp [202], [203].

5. Representations over residue class rings $Z / m Z$ : DrobotenkoDrobotenko-Žilinskaja-Pogoriljak [66], Drobotenko-Gudivok-Lihtman [68], Drobotenko-Lihtman [67], Hannula [103], NazarovaRoĭter [169].

6. Representations of orders in algebras: Bass [11], BorevičFaddeev [34], [35], Drozd [70], Drozd-Kiričenko [71], DrozdKiričenko-Roǔter [73], Drozd-Roĭter [74], Drozd-Turčin [75], Faddeev [81], [83], Jacobinski [127], Kaplansky [140], Kiričenko [141], Nazarova [166], Nazarova-Roŭter [170], [171].

16. Representation rings. Let $R$ be a Dedekind domain whose quotient field $F$ is an algebraic number field, and let $\Lambda$ be an $R$ order in the semisimple $F$-algebra $A$. By the representation group of $\Lambda$ we shall mean the abelian additive group $a(\Lambda)$ generated by the symbols $[M]$, one for each isomorphism class of $\Lambda$-lattices, and with relations $[M]=\left[M^{\prime}\right]+\left[M^{\prime \prime}\right]$ whenever $M \cong M^{\prime}+M^{\prime \prime}$. If $M$ and $N$ are $\Lambda$-lattices then $[M]=[N]$ in $a(\Lambda)$ if and only if $M+X \cong N+X$ for some $\Lambda$-lattice $X$.

(Note that $a(\Lambda)$ is just the Grothendieck group $K(\mathfrak{e}, s)$ of $\$ 10$ where $\mathcal{C}$ is the category of all left $\Lambda$-lattices and $S$ the collection of all split short exact sequences from $\mathcal{C}$.) 
Let us consider the additive homomorphism

$$
\phi: a(\Lambda) \rightarrow \sum_{P}^{\cdot} a\left(\Lambda_{P}^{*}\right),
$$

where $P$ ranges over all maximal ideals of $R$ containing the Higman ideal $i(\Lambda)$ defined in $\$ 4$.

(16.1). TheOREM (REINer [195]). The kernel of $\phi$ is precisely the torsion submodule of the additive group $a(\Lambda)$, and may be characterized as the finite group consisting of all elements

$$
\{[\Lambda]-[M]: M \in \text { genus of } \Lambda\} \text {. }
$$

As in (6.2), we introduce the semilocal ring $\widetilde{R}$ defined by

$$
\widetilde{R}=\bigcap_{P \supset i(\Lambda)} R_{P}
$$

and set $\tilde{\Lambda}=\tilde{R} \Lambda$. Then the map $\phi$ factors through $a(\tilde{\Lambda})$, that is, we may write

$$
a(\Lambda) \underset{\mu}{\rightarrow} a(\tilde{\Lambda}) \underset{\phi^{\prime}}{\rightarrow} \sum_{P \supset i(\Lambda)} a\left(\Lambda_{P}^{*}\right) .
$$

Here $\mu$ and $\phi^{\prime}$ are homomorphisms with $\phi^{\prime} \cdot \mu=\phi$, and $\mu$ is epic, $\phi^{\prime}$ is monic. The additive group $a(\tilde{\Lambda})$ is generated by indecomposable genera; these need not form a free $Z$-basis of $a(\tilde{\Lambda})$, however, since the Krull-Schmidt Theorem is not generally true for $\tilde{\Lambda}$-lattices (see $\$ 19$ ). Nevertheless, $a(\tilde{\Lambda})$ is $Z$-free, since $\phi^{\prime}$ embeds it in a $Z$-free module.

More generally let $\beta$ be some category of left $\Lambda$-lattices closed under formation of direct sums, and let $\delta$ be the collection of all split short exact sequences from $B$. The relative Grothendieck group $K(ß, S)$ will be denoted briefly by $a(B)$. Just as above we may consider the map

$$
\phi: a(B) \rightarrow \sum_{P} \cdot a\left(\left(_{P}^{*}\right)\right.
$$

where now $P$ ranges over some nonempty finite set of maximal ideals of $R$, including all those for which $\Lambda_{P}^{*}$ is not a maximal order. Jacobinski [129] showed that the kernel of $\phi$ is precisely the torsion subgroup of $a(B)$, and can be given explicitly as a subgroup of $V(M, \mathfrak{f})$ for some suitably chosen $\Lambda$-lattice $M$ in the category $B$. (Here, $V(M, \mathfrak{f})$ is as defined in (8.7).)

(16.2). Theorem (Jacobinski [129]). Let $M$ be some fixed $\Lambda$-lattice, 
and choose $B$ to be the category of all $\Lambda$-lattices which are direct summands of $M^{(n)}$ for some $n$. Then (see $\$ 8$ for notation):

(i) $\operatorname{ker} \phi \cong V\left(M^{(t)}, \mathfrak{f}\right)$, where $t=1$ if $M$ satisfies the Eichler condition, while $t=2$ otherwise.

(ii) The image of $\phi$ is a finitely generated free $Z$-module.

Remark. Theorem 14.9 follows readily from (ii). On the other hand, Theorem 10.14 is the special case of (i) in which $M=\Lambda$.

When $\Lambda=R G$, we can make $a(\Lambda)$ into a commutative ring, defining multiplication by $[M][N]=\left[M \otimes_{R} N\right]$, with the elements of $G$ acting diagonally on $M \otimes N$. It follows at once from (16.1) that $(\operatorname{ker} \phi)^{2}$ $=0$. On the other hand, while both $a\left(R_{P} G\right)$ and $a\left(R_{P}^{*} G\right)$ are free $Z$-modules, very little is known about their multiplicative structure. $\mathrm{Up}$ to this point, attention has centered on the question as to whether $a\left(R_{P} G\right)$ can contain any nonzero nilpotent elements; the corresponding question for $a(k G)$, where $k$ is a modular field, has been only partially settled. $\dagger$

(16.3). Theorem (ReINer [190]-[192]). Let $R_{P}$ be a valuation ring in $F$, and let $G$ contain a cyclic subgroup of order $n$, where $n \in P^{2}$; and if $2 \in P$ assume further that $n \in 2 P$. Then $a\left(R_{P} G\right)$ and $a\left(R_{P}^{*} G\right)$ contain nonzero nilpotent elements.

On the other hand, suppose that $[G: 1]$ is squarefree. Then $a\left(Z_{p} G\right)$ and $a\left(Z_{p}^{*} G\right)$ contain no nonzero nilpotent elements.

Rud'ko [219] has determined the multiplication table for the ring $a\left(Z_{p}^{*} G\right)$, where $G$ is cyclic of order $p^{2}$.

Complementing the preceding theorem, we quote

(16.4). Theorem (Zemanek [265]). If $G$ has a noncyclic Sylow p-subgroup, then $a(Z G), a\left(Z_{p} G\right)$ and $a\left(Z_{p}^{*} G\right)$ all contain nonzero nilpotent elements.

17. Group rings. Let $G, H$ be finite groups, and $K$ a field. It is known that the group algebra $K G$ need not determine the group $G$ up to isomorphism. Thus, for example, Berman [20] proved that if $p$ is an odd prime, and $G, H$ are nonisomorphic noncommutative groups of order $p^{3}$, then $Q G \cong Q H$. On the other hand, Passman [178] showed that if $K$ is a field of characteristic $p$, there exist many nonisomorphic groups $G, H$ with $K G \cong K H$. For a survey of the subject of group algebras, we refer the reader to Holvoet [124].

Now let $R=$ alg. int. $\{F\}$. The following problem is still unsettled:

$\dagger$ Using the results of [265], Zemanek has recently shown that $a(k G)$ contains nonzero nilpotent elements whenever char $k=p>2$, and $G$ is an abelian group of type $(p, p)$. 
If $R G \cong R H$, does it necessarily follow that $G \cong H$ ? Partial answers so far obtained are given below:

(17.1). Theorem. If $G, H$ are abelian and $Z G \cong Z H$, then $G \cong H$.

References: G. Higman [123], CR $\$ 37$.

(17.2). Theorem (MAY [160]). If $Z G \cong Z H$, then $G$ and $H$ have isomorphic commutator factor groups.

(17.3).THEOREM. If $G, H$ are metabelian groups, then $Z G \cong Z H$ implies that $G \cong H$.

References: Jackson [124b], [124c], Whitcomb [261].

(17.4). Theorem (Passman [178]). Let $R=$ alg. int. $\{F\}$. The integral group ring $R G$ uniquely determines the lattice of normal subgroups of $G$, and also the upper and lower central series of $G$. If $Z_{1}(G)$ is the center of $G$, and $Z_{2}(G) / Z_{1}(G)$ is the center of $G / Z_{1}(G)$, then $R G \cong R H$ implies that $Z_{2}(G) \cong Z_{2}(H)$. Hence if $G$ is nilpotent of class 2 , and $R G \cong R H$, then $G \cong H$.

(17.5). Corollary. If $G / Z_{1}(G)$ is a p-group of order at most $p^{3}$, then $R G \cong R H$ implies that $G \cong H$.

The article by Passman [178] contains many other results of this nature.

Also of interest is the question of determining the units in the group ring $R G$.

(17.6). Theorem. Let $R=$ alg. int. $\{F\}$. Any unit of finite order in $R G$ is of the form $\epsilon x$, where $x \in G$ and $\epsilon$ is a root of unity in $R$.

References. G. Higman [123], CR \$37.

For further discussion of the units in $R G$, and their significance for algebraic $K$-theory, we refer the reader to Bass [14, Chapter 11, $\$ 7]$.

Let $I$ denote the augmentation ideal of $Z G$, that is,

$$
I=\sum \cdot Z(x-1)
$$

where $x$ ranges over all elements of $G$ except 1 . Gruenberg [94] proved:

$$
\bigcap_{n=1}^{\infty} I^{n}=0 \text { if and only if } G \text { is a } p \text {-group. }
$$

For other results of this nature, as well as for a general algebraic study of group rings, see Connell [45], Passman [177], [178]. 
Further references on group rings: Banaschewski [5], Berman [16]-[20], Bovdi [37], Cohn-Livingstone [42], [43], Larson [147], Saksonov [220], [221], Schneider-Weissglass [223], Sehgal [224], Takahashi [239].

18. Algebraic number theory. Clearly the methods of algebraic number theory play a large role in the study of integral representations of orders. Recently, however, representation theory has begun to yield results in algebraic number theory. The chief application deals with the following problem:

Let $R$ be a Dedekind domain with quotient field $F$, and let $F^{\prime}$ be a finite normal separable field extension of $F$, with Galois group $G$. Denote by $R^{\prime}$ the integral closure of $R$ in $F^{\prime}$. Then the elements of $G$ act on $R^{\prime}$, and $R^{\prime}$ is an $R G$-lattice. What can be said about $R^{\prime}$ as $R G$-lattice?

More generally, an $R^{\prime}$-ideal $J$ in $F^{\prime}$ is ambiguous if $\sigma(J)=J$ for all $\sigma \in G$. Each ambiguous ideal $J$ in $F^{\prime}$ is also an $R G$-lattice, and $J$ is $R G$-free if and only if $J$ has a normal integral basis over $R$ (that is, $J=\sum_{\sigma \in G} R \cdot \sigma(x)$ for some $\left.x \in J\right)$. In particular, then, $R^{\prime}$ has a normal integral basis over $R$ if and only if $R^{\prime} \cong R G$ as $R G$-modules.

(i) Let $R=$ alg. int. $\{F\}$. Call $F^{\prime}$ tamely ramified over $F$ if no maximal ideal of $R$ divides the different of $F^{\prime}$ over $F$, or equivalently, if some element of $R^{\prime}$ has trace equal to 1 . In this case, every ambiguous ideal is $R G$-projective; conversely, if $R^{\prime}$ is $R G$-projective, then $F^{\prime}$ is tamely ramified over $F$.

(ii) Hilbert proved that if $F^{\prime}$ is an abelian extension of the rational field $Q$, then $R^{\prime} \cong Z G$ if and only if $F^{\prime}$ is tamely ramified over $Q$. (See Ullom [256], [257] for a partial generalization to the case of ambiguous ideals in $R^{\prime}$.)

(iii) Fröhlich [86] gave a necessary and sufficient condition for the isomorphism $R^{\prime} \cong R G$, assuming that $F^{\prime}$ is an abelian extension of $F$ of a suitably restricted type (Kummer extension).

(iv) Leopoldt [151] showed that when $F^{\prime}$ is an abelian extension of $Q$, then

$$
O_{l}\left(R^{\prime}\right)=\left\{x \in Q G: x \cdot R^{\prime} \subset R^{\prime}\right\}
$$

is isomorphic to $R^{\prime}$ as left $Z G$-module. Further, $O_{l}\left(R^{\prime}\right)$ is an order in $Q G$ which can be obtained from $Z G$ by adjunction of certain idempotents arising from wild ramification. If $F^{\prime}$ is tamely ramified over $Q$, then indeed $O_{l}\left(R^{\prime}\right)=Z G$, and we recover Hilbert's result (ii). Jacobinski [125] investigated $O_{l}\left(R^{\prime}\right)$ in more general circumstances.

(v) Martinet [155a] recently proved that if $F$ is a normal tamely 
ramified extension of $Q$ whose Galois group $G$ is dihedral of order $2 p$ ( $p$ an odd prime), then alg. int $\{F\} \cong Z G$, so a normal integral basis exists in this case.

Other references for this section: Newman-Taussky [173].

19. Krull-Schmidt and Cancellation Theorems. Let $R$ be a Dedekind domain, $\Lambda$ any $F$-algebra. We shall say that the KrullSchmidt Theorem is valid for $\Lambda$-lattices if every $\Lambda$-lattice is expressible as a direct sum of indecomposable lattices which are uniquely determined up to $\Lambda$-isomorphism and order of occurrence. As pointed out in Theorems 5.2 and 5.8, we have

(19.1). THEOREM. The Krull-Schmidt theorem holds for $\Lambda$-lattices if

(i) $R$ is a complete discrete valuation ring, or

(ii) $R$ is a discrete valuation ring, and $A$ is a direct sum of full matrix algebras over $F$, or

(iii) $R$ is a discrete valuation ring and $A$ is a direct sum of full matrix algebras over skerwfields which remain skewfields upon passing from $F$ to its completion.

When $R=$ alg. int. $\{F\}$, the Krull-Schmidt Theorem holds for $R$-lattices if and only if $R$ is a principal ideal domain. Hence in order to insure that the Krull-Schmidt Theorem is valid for $\Lambda$-lattices, it is usually necessary to impose strong restrictions on $R$. Often one deals instead with the easier case of $R_{P}$-lattices.

Let $Z_{p}$ denote the localization of $Z$ at the prime ideal $(p)$ of $Z$. Berman-Gudivok [27] observed that the Krull-Schmidt Theorem already fails for $Z_{p} G$-lattices when $G$ is a suitably chosen cyclic group of squarefree order. In this direction we state

(19.2). Theorem (Jones [137]). Let $G$ be an abelian group of exponent $k \cdot p^{n}$ where $p$ is a prime, $p \nmid k$. The Krull-Schmidt Theorem holds for $Z_{p} G$-lattices if and only if one of the following holds true:

(i) $k=1$.

(ii) $n=0$.

(iii) The element $p$ generates the multiplicative group of integers (mod $k$ ) which are relatively prime to $k$.

Other references. Dress [58], Roggenkamp [206].

Now let $A$ be a separable algebra over $F$, and let $i(\Lambda)$ be the Higman ideal of $\Lambda$ (see $\S 4$ ) (so $i(\Lambda)=[G: 1] R$ when $\Lambda=R G$ ). As in $\S 6$, define

$$
\widetilde{R}=\bigcap_{\boldsymbol{P}} R_{P},
$$

where $P$ ranges over all maximal ideals of $R$ containing $i(\Lambda)$. Set 
$\tilde{\Lambda}=\widetilde{R} \Lambda$, an $\widetilde{R}$-order in $A$ useful in the study of genera. As shown by Reiner [188], the Krull-Schmidt Theorem need not hold for $\dot{\Lambda}$-lattices; indeed, the $\widetilde{R}$-ranks of the indecomposable direct summands of a $\tilde{\Lambda}$-lattice $\tilde{M}$ need not be uniquely determined by $\tilde{M}$. This question as to when the Krull-Schmidt Theorem holds for $\tilde{\Lambda}$-lattices is studied in detail in Jacobinski [129].

Generalizing Theorem 5.8, we have

(19.3). THEOREM (JACOBINSKI [129]). Let $F$ be an algebraic number field, and let $\Lambda$ be an $R$-order in the semisimple F-algebra $A$. Suppose there exists a maximal ideal $P$ of $R$ such that

(i) for every maximal ideal $P^{\prime}$ of $R$ distinct from $P$, the order $\Lambda_{P^{\prime}}$ is a maximal $R_{P^{\prime} \text {-order in } A \text {, and }}$

(ii) every $A_{P}^{*}$-module is of the form $F_{P}^{*} \otimes_{F} X$ for some $A$-module $X$.

Then the Krull-Schmidt Theorem is valid for $\tilde{\Lambda}$-lattices (and for $\Lambda_{P}$-lattices as well).

(19.4). Corollary. Let $G$ be a finite $p$-group, where $p \neq 2, p$ prime. Then the Krull-Schmidt Theorem holds for $Z_{p} G$-lattices.

We shall say that the Cancellation Theorem holds for $\Lambda$-lattices if for each triple of $\Lambda$-lattices $M, N, X$, the isomorphism $M \dot{+} X \cong N \dot{+} X$ implies that $M \cong N$. Of course, if the Krull-Schmidt Theorem holds for $\Lambda$-lattices, then so does the Cancellation Theorem. When $R=$ alg. int. $\{F\}$, the Cancellation Theorem need not be true for $R G$ lattices; see for example Berman-Gudivok [27], as well as Theorem 8.12 above.

Indeed, an important example shows that the Cancellation Theorem is not necessarily true for the category of projective $R G$ lattices. We quote

(19.5). Theorem (SwAN [233]). Let $G$ be a generalized quaternion group of order 32 . There exists a projective left ideal $M$ in the group ring $Z G$, such that

$$
Z G+Z G \cong M+Z G, \quad M \nsubseteq Z G .
$$

In fact, if $\Gamma$ is a maximal $Z$-order in $Q G$ containing $Z G$, a corresponding result holds for $\Gamma$-lattices.

Given $\Lambda$-lattices $M$ and $N$, we have called $N$ a local summand of $M$ if $N_{P}^{*} \mid M_{P}^{*}$ for each maximal ideal $P$ of $R$.

(19.6). THEOREM (JACOBINSKI [129]). Let $F$ be an algebraic number field, and let $\Lambda$ be an $R$-order in the semisimple F-algebra A. Suppose that $M$ and $N$ are left $\Lambda$-lattices which satisfy the Eichler condition (see 
$\S 8)$, and let the $\Lambda$-lattice $X$ be a local summand of $M^{(n)}$ for some $n$. Then $M+X \cong N+X$ implies that $M \cong N$.

(19.7). Corollary (Bass $[12, \S 9])$. Let $X$ be a projective $\Lambda$-lattice, and $M$ any $\Lambda$-lattice having $\Lambda^{(2)}$ as local summand. Then for any $\Lambda$ lattice $N, M+X \cong N+X$ implies that $M \cong N$.

Finally we note

(19.8). Theorem (J ACOBINski [129]). Let $F$ be an algebraic number field, and let $G$ be a finite group such that no prime divisor of $[G: 1]$ is a unit in $R$. Suppose that no simple component of the group algebra $F G$ is a totally definite quaternion algebra. ${ }^{15}$ Then the Cancellation Theorem holds in the category of projective RG-lattices.

\section{REFERENCES}

1. M. Auslander and O. Goldman, Maximal orders, Trans. Amer. Math. Soc. 97 (1960), 1-24. MR 22 \#8034.

2. - The Brauer group of a commutative ring, Trans. Amer. Math. Soc. 97 (1960), 367-409. MR 22 \#12130.

3. G. Azumaya, Corrections and supplementaries to my paper concerning KrullRemak-Schmidt's theorem, Nagoya Math. J. 1 (1950), 117-124. MR 12, 314.

4. D. Ballew, The module index, projective modules and invertible ideals, Ph.D. Thesis, University of Illinois, Urbana, Ill., 1969.

4a. - - The module index and invertible ideals, Trans. Amer. Math. Soc. 148 (1970), (to appear).

5. B. Banaschewski, Integral group rings of finite groups, Canad. Math. Bull. 10 (1967), 635-642. MR 38 \#1187.

6. L. F. Barannik and P. M. Gudivok, Projective representations of finite groups over rings, Dopovīdi Akad. Nauk Ukraïn. RSR Ser. A 1968, 294-297. (Ukrainian) MR 37 \#4177.

7. - On indecomposable projective representations of finite groups, Dopovidi Akad. Nauk Ukrain. RSR Ser. A 1969, 391-393. (Ukrainian)

8. H. Bass, Finitistic dimension and a homological generalization of semi-primary rings, Trans. Amer. Math. Soc. 95 (1960), 466-488. MR 28 \#1212.

9. - Projective modules over algebras, Ann. of Math. (2) 73 (1961), 532-542. MR 31 \#1278.

10. - Torsion free and projective modules, Trans. Amer. Math. Soc. 102 (1962), 319-327. MR 25 \#3960.

11. - On the ubiquity of Gorenstein rings, Math. Z. 82 (1963), 8-28. MR 27 \#3669.

12. - K-theory and stable algebra, Inst. Hautes Etudes Sci. Publ. Math. No. 22 (1964), 5-60. MR 30 \#4805.

13. - The Dirichlet unit theorem, induced characters, and Whitehead groups of finite groups, Topology 4 (1966), 391-410. MR 33 \#1341. 1968.

14. —, Algebraic K-theory, Math. Lecture Note Series, Benjamin, New York,

${ }^{15}$ See end of $\$ 7$. 
15. E. A. Bender, Classes of matrices and quadratic fields, Linear Algebra and Appl. 1 (1968), 195-201. MR 37 \#6301.

16. S. D. Berman, On certain properties of integral group rings, Dokl. Akad. Nauk SSSR 91 (1953), 7-9. (Russian) MR 15, 99.

17. - On isomorphism of the centers of group rings of p-groups, Dokl. Akad. Nauk SSSR 91 (1953), 185-187. (Russian) MR 15, 99.

18. - On a necessary condition for isomorphism of integral group rings, Dopovīdī Akad. Nauk Ukraïn. RSR 1953, 313-316. (Ukrainian) MR 15, 599.

19. - On the equation $x^{m}=1$ in an integral group ring, Ukrain. Mat. Ž. 7 (1955), 253-261. (Russian) MR 17, 1048.

20. - On certain properties of group rings over the field of rational numbers, Užgorod. Gos. Univ. Naučn. Zap. Him. Fiz. Mat. 12 (1955), 88-110. (Russian) MR $20 \# 3920$.

21. - On automorphisms of the center of an integral group ring, Užgorod. Gos. Univ. Naučn. Zap. Him. Fiz. Mat. (1960), no. 3, 55. (Russian)

22. - Integral representations of finite groups, Dokl. Akad. Nauk SSSR 152 (1963), 1286-1287= Soviet Math. Dokl. 4 (1963), 1533-1535. MR 27 \#4854.

23. - On the theory of integral representations of finite groups, Dokl. Akad. Nauk SSSR 157 (1964), 506-508=Soviet Math. Dok1. 5 (1964), 954-956. MR 29 \#2308.

24. - Integral representations of a cyclic group containing two irreducible rational components, In Memoriam: N. G. Čebotarev, Izdat. Kazan Univ., Kazan, 1964, pp. 18-29. (Russian) MR 33 \#4154.

25. - On integral monomial representations of finite groups, Uspehi Mat. Nauk 20 (1965), no. 4 (124), 133-134. (Russian) MR 33 \#4155.

26. - Representations of finite groups over an arbitrary field and over rings of integers, Izv. Akad. Nauk SSSR Ser. Mat. 30 (1966), 69-132; English transl., Amer. Math. Soc. Transl. (2) 64 (1967), 147-215. MR 33 \#5747.

27. S. D. Berman and P. M. Gudivok, Integral representations of finite groups, Dokl. Akad. Nauk SSSR 145 (1962), 1199-1201 = Soviet Math. Dokl. 3 (1962), 11721174. MR $25 \# 3095$.

28. - Integral representations of finite groups, Užgorod. Gos. Univ. Naučn. Zap. Him. Fiz. Mat. (1962), no. 5, 74-76. (Russian)

29. - Indecomposable representations of finite groups over the ring of p-adic integers, Izv. Akad. Nauk SSSR Ser. Mat. 28 (1964), 875-910; English transl., Amer. Math. Soc. Transl. (2) 50 (1966), 77-113. MR 29 \#3550.

30. S. D. Berman and A. I. Lihtman, On integral representations of finite nilpotent groups, Uspehi Mat. Nauk 20 (1965), no. 5 (125), 186-188. (Russian) MR 34 \#7673.

31. S. D. Berman and A. R. Rossa, On integral group-rings of finite and periodic groups, Algebra and Math. Logic: Studies in Algebra, Izdat. Kiev Univ., Kiev, 1966, pp. 44-53. (Russian) MR 35 \#265.

31a. A. Bialnicki-Birula, On the equivalence of integral representations of finite groups, Proc. Amer. Math. Soc. (to appear).

32. Z. I. Borevic and D. K. Faddeev, Theory of homology in groups. I, II, Vestnik Leningrad. Univ. 11 (1956), no. 7, 3-39; 14 (1959), no. 7, 72-87. (Russian) MR 18, 188; MR 21 \#4968.

33. - Integral representations of quadratic rings, Vestnik Leningrad. Univ. 15 (1960), no. 19, 52-64. (Russian) MR 27 \#3668.

34. - Representations of orders with cyclic index, Trudy Mat. Inst. Steklov. 80 (1965), 51-65. Proc. Steklov Inst. Math. 80 (1965), 56-72. MR 34 \#5805.

35. - Remarks on orders with a cyclic index, Dokl. Akad. Nauk SSSR 164 (1965), 727-728=Soviet Math. Dokl. 6 (1965), 1273-1274. MR 32 \#7601. 
36. N. Bourbaki, Algèbre commutative, Actualités Sci. Indust., no. 1293, Hermann, Paris, 1961. MR 30 \#2027.

37. A. A. Bovdi, Periodic normal divisors of the multiplicative group of a group ring, Sibirsk Mat. Ž. 9 (1968), 495-498 = Siberian Math. J. 9 (1968), 374-376. MR 37 \#2853.

38. J. O. Brooks, Classification of representation modules over quadratic orders, Ph.D. Thesis, University of Michigan, Ann Arbor, Mich., 1964.

39. A. Brumer, Structure of hereditary orders, Bull. Amer. Math. Soc. 69 (1963), 721-724; Addendum, ibid. 70 (1964), 185. MR 27 \#2543.

40. H. Cartan and S. Eilenberg, Homological algebra, Princeton Univ. Press, Princeton, N. J., 1956. MR 17, 1040.

41. C. Chevalley, L'arithmétique dans les algèbres de matrices, Actualités Sci. Indust., no. 323, Hermann, Paris, 1936.

42. J. A. Cohn and D. Livingstone, On groups of order $p^{3}$, Canad. J. Math. 15 (1963), 622-624. MR $27 \# 3700$.

43. - On the structure of group algebras, Canad. J. Math. 17 (1965), 583-593. MR 31 \#3514.

44. D. B. Coleman, Idempotents in group rings, Proc. Amer. Math. Soc. 17 (1966), 962. MR 33 \#1379.

44a. S. B. Conlon, Structure in representation algebras, J. Algebra 5 (1967), 274279. MR 34 \#2719.

44b. - Relative components of representations, J. Algebra 8 (1968), 478-501.

44c. - Decompositions induced from the Burnside algebra, J. Algebra 10 (1968), 102-122. MR 38 \#5945.

44d. - Monomial representations under integral similarity, J. Algebra 13 (1969), 496-508.

45. I. G. Connell, On the group ring, Canad. J. Math. 15 (1963), 650-685. MR 27 \#3666.

46. C. W. Curtis and I. Reiner, Representation theory of finite groups and associative algebras, Pure and Appl. Math., vol. XI, Interscience, New York, 1962; 2nd ed., 1966. MR 26 \#2519.

47. E. C. Dade, Rings in which no fixed power of ideal classes becomes invertible, Math. Ann. 148 (1962), 65-66. MR 25 \#3963.

48. - Some indecomposable group representations, Ann. of Math. (2) 77 (1963), 406-412. MR 26 \#2521.

49. - The maximal finite groups of $4 \times 4$ integral matrices, Illinois J. Math. 9 (1965), 99-122. MR 30 \#1192.

50. E. C. Dade, D. W. Robinson, O. Taussky, and M. Ward, Divisors of recurrent sequences, J. Reine Angew. Math. 214/215 (1964), 180-183. MR 28 \#5079.

51. E. C. Dade and O. Taussky, Some new results connected with matrices of rational integers, Proc. Sympos. Pure Math., vol. 8, Amer. Math. Soc., Providence, R. I., 1965, pp. 78-88. MR 32 \#2395.

51a. - On the different in orders in an algebraic number field and special units connected with it, Acta Arith. 9 (1964), 47-51.

52. E. C. Dade, O. Taussky and H. Zassenhaus, On the semigroup of ideal classes in an order of an algebraic number field, Bull. Amer. Math. Soc. 67 (1961), 305-308. MR 25 \#65.

53. - On the theory of orders, in particular on the semigroup of ideal classes and genera of an order in an algebraic number field, Math. Ann. 148 (1962), 31-64. MR 25 $\# 3962$.

54. K. deLeeuw, Some applications of cohomology to algebraic number theory and group representations (unpublished). 
55. F. R. DeMeyer, The trace map and separable algebras, Osaka J. Math. 3 (1966), 7-11. MR $37 \# 4122$.

56. M. Deuring, Algebren, Springer-Verlag, Berlin, 1935; rev. ed., Ergebnisse der Mathematik und ihrer Grenzgebiete, Band 41, 1968. MR 37 \#4106.

57. F. E. Diederichsen, Über die Ausreduktion ganzzahliger Gruppendarstellungen bei arithmetischer Aquivalenz, Abh. Math. Sem. Univ. Hamburg 14 (1938), 357-412.

58. A. Dress, A remark on the Krull-Schmidt theorem for integral group representations of rank 1 (to appear).

59. - - An intertwining number theorem for integral representations and applications (to appear).

60. - On the decomposition of modules, Bull. Amer. Math. Soc. 75 (1969), 984-986. 1034.

61. - On integral representations, Bull. Amer. Math. Soc. 75 (1969), 1031-

62. - The ring of monomial representations. I: Structure Theory (to appear).

63. - Vertices of integral representations, Math. Z. (to appear).

64. - On relative Grothendieck rings, Bull. Amer. Math. Soc. 75 (1969), 955-958.

65. V. S. Drobotenko, Integral representations of primary abelian groups, Algebra and Math. Logic: Studies in Algebra, Izdat. Kiev. Univ., Kiev, 1966, pp. 111-121. (Russian) MR 34 \#4375.

66. V. S. Drobotenko, E. S. Drobotenko, Z. P. Žilinskaja and E. Y. Pogoriljak, Representations of the cyclic group of prime order $p$ over residue classes mod $p^{s}$, Ukrain. Mat. Ž. 17 (1965), no. 5, 28-42; English transl., Amer. Math. Soc. Transl. (2) 69 (1968), 241-256. MR 32 \#5743.

67. V. S. Drobotenko and A. I. Lihtman, Representations of finite groups over the ring of residue classes mod $p^{s}$, Dokl. Užgorod Univ. 3 (1960), 63. (Russian)

68. V. S. Drobotenko, P. M. Gudivok and A. I. Lihtman, On representations of finite groups over the ring of residue classes mod $m$, Ukrain. Mat. Ž. 16 (1964), 82-89. (Russian) MR $29 \# 4810$.

69. V. S. Drobotenko and V. P. Rud'ko, Representations of a cyclic group by groups of automorphisms of a certain class of modules, Dopovidi Akad. Nauk Ukrain. RSR Ser. A. 1968, 302-304. (Ukrainian) MR 37 \#2873.

70. Ju. A. Drozd, Representations of cubic Z-rings, Dokl. Akad. Nauk SSSR 174 (1967), 16-18= Soviet Math. Dokl. 8 (1967), 572-574. MR 35 \#6659. 19-24.

70a. - On the distribution of maximal sublattices, Mat. Zametki 6 (1969),

70b. - - Adeles and integral representations, Izv. Akad. Nauk SSSR Ser. Mat. 33 (1969), 1080-1088.

71. Ju. A. Drozd, and V. V. Kiričenko, Representation of rings in a second order matrix algebra, Ukrain. Mat. Ž. 19 (1967), no. 3, 107-112. (Russian) MR 35 \#1632.

72. - Hereditary orders, Ukrain. Mat. Ž. 20 (1967), 246-248. (Russian).

73. Ju. A. Drozd, V. V. Kirǐenko and A. V. Rơ̌ter, On hereditary and Bass orders, Izv. Akad. Nauk SSSR Ser. Mat. 31 (1967), 1415-1436 = Math. USSR Izv. 1 (1967), 1357-1376. MR 36 \#2608.

74. Ju. A. Drozd and A. V. Royter, Commutative rings with a finite number of indecomposable integral representations, Izv. Akad. Nauk SSSR Ser. Mat. 31 (1967), 783-798 = Math. USSR Izv. 1 (1967), 757-772. MR 36 \#3768.

75. Ju. A. Drozd and V. M. Turčin, Number of representation modules in a genus 
for integral second order matrix rings, Mat. Zametki 2 (1967), 133-138= Math. Notes 2 (1967), 564-566. MR 37 \#5253.

76. V. H. Dyson and K. W. Roggenkamp, Modules over orders, Springer Lecture Notes (to appear).

77. M. Eichler, Über die Idealklassenzahl total definiter Quaternionenalgebren, Math. Z. 43 (1938), 102-109.

78. — Über die Idealklassenzahl hyperkomplexer Zahlen, Math. Z. 43 (1938), $481-494$.

79. D. K. Faddeev, On the semigroup of genera in the theory of integer representations, Izv. Akad. Nauk SSSR Ser. Mat. 28 (1964), 475-478; English transl., Amer. Math. Soc. Transl. (2) 64 (1967), 97-101. MR 28 \#5089.

80. - An introduction to multiplicative theory of modules of integral representations, Trudy Mat. Inst. Steklov. 80 (1965), 145-182 = Proc. Steklov Inst. Math. 80 (1965), 164-210. MR 34 \#5873.

81. - On the theory of cubic Z-rings, Trudy Mat. Inst. Steklov. 80 (1965), 183-187 = Proc. Steklov Inst. Math. 80 (1965), 211-215. MR 33 \#4083.

82. — - Equivalence of systems of integer matrices, Izv. Akad. Nauk SSSR Ser. Mat. 30 (1966), 449-454; English transl., Amer. Math. Soc. Transl. (2) 71 (1968), 43-48. MR 33 \#2642.

83. - The number of classes of exact ideals for Z-rings, Mat. Zametki 1 (1967), 625-632 = Math. Notes 1 (1967), 415-419. MR 35 \#5466.

84. R. Fossum, The Noetherian different of projective orders, J. Reine Angew. Math. 224 (1966), 207-218. MR 36 \#5119.

84a. - Maximal orders over Krull domains, J. Algebra 10 (1968), 321-332. MR $38 \# 2130$.

85. A. Fröhlich, Ideals in an extension field as modules over the algebraic integers in a finite number field, Math. Z. 74 (1960), 29-38. MR 22 \#4708.

86. - The module structure of Kummer extensions over Dedekind domains,

J. Reine Angew. Math. 209 (1962), 39-53. MR 28 \#3988; p. 1247.

87. - Invariants for modules over commutative separable orders, Quart. J. Math. Oxford Ser. (2) 16 (1965), 193-232. MR $35 \# 1583$.

88. - Resolvents, discriminants, and trace invariants, J. Algebra 4 (1966), 173-198. MR 34 \#7499.

89. I. Giorgiutti, Modules projectifs sur les algèbres de groupes finis, C.R. Acad. Sci. Paris 250 (1960), 1419-1420. MR 23 \#A1691.

90. J. A. Green, On the indecomposable representations of a finite group, Math. Z. 70 (1958/59), 430-445. MR 24 \#A1304.

91. — Blocks of modular representations, Math. Z. 79 (1962), 100-115. MR $25 \# 5114$.

92. - The modular representation algebra of a finite group, Illinois J. Math. 6 (1962), 607-619. MR 25 \#5106.

93. - A transfer theorem for modular representations, J. Algebra 1 (1964), 73-84. MR 29 \#147.

94. K. Gruenberg, Residual properties of infinite soluble groups, Proc. London Math. Soc. (3) 7 (1957), 29-62. MR 19, 386.

95. P. M. Gudivok, Integral representations of a finite group with a noncyclic Sylow p-subgroup, Uspehi Mat. Nauk 16 (1961), 229-230.

96. - Integral representations of groups of type $(p, p)$, Dokl. Užgorod Univ. Ser. Phys.-Mat. Nauk (1962), no. 5, 73. 
97. - On p-adic integral representations of finite groups, Dokl. Užgorod Univ. Ser. Phys.-Mat. Nauk (1962), no. 5, 81-82.

98. - Representations of finite groups over certain local rings, Dopovid Akad. Nauk Ukraïn. RSR 1964, 173-176. (Ukrainian) MR 29 \#3551.

99. - Representations of finite groups over quadratic rings, Dokl. Akad. Nauk SSSR 159 (1964), 1210-1213 =Soviet Math. Dokl. 5 (1964), 1669-1672. MR 30 $\# 174$.

100. - Representations of finite groups over local number rings, Dopovidi Akad. Nauk Ukrain. RSR 1966, 979-981. (Ukrainian) MR 34 \#1407.

101. - Representations of finite groups over number rings, Izv. Akad. Nauk SSSR Ser. Mat. 31 (1967), 799-834=Mat. USSR Izv. 1 (1967), 773-805. MR 36 \#1554.

102. P. M. Gudivok and V. P. Rud'ko, On p-adic integer-valued representations of a cyclic p-group, Dopovidi Akad. Nauk Ukrain. RSR 1966, 1111-1113. (Ukrainian) MR 34 \#1409.

103. T. Hannula, The integral representation ring $a\left(R_{k} G\right)$, Trans. Amer. Math. Soc. 133 (1968), 553-559.

104. M. Harada, Hereditary orders, Trans. Amer. Math. Soc. 107 (1963), 273-290. MR 27 \#1474.

105. - Structure of hereditary orders over local rings, J. Math. Osaka City Univ. 14 (1963), 1-22. MR 29 \#5879.

106. - Multiplicative ideal theory in hereditary orders, J. Math. Osaka City Univ. 14 (1963), 83-106. MR 29 \#5880b.

107. A. Hattori, Rank element of a projective module, Nagoya Math. J. 25 (1965), 113-120. MR 31 \#226.

108. - Semisimple algebras over a commutative ring, J. Math. Soc. Japan 15 (1963), 404-419. MR 28 \#2125.

109. A. Heller, On group representations over a valuation ring, Proc. Nat. Acad. Sci. U.S.A. 47 (1961), 1194-1197. MR 23 \#A2468.

110. - Some exact sequences in algebraic K-theory, Topology 3 (1965), 389408. MR $31 \# 3477$.

111. A. Heller and I. Reiner, Indecomposable representations, Illinois J. Math. 5 (1961), 314-323. MR 23 \#A222.

112. - Representations of cyclic groups in rings of integers. I, II, Ann. of Math. (2) 76 (1962), 73-92; (2) 77 (1963), 318-328. MR 25 \#3993; MR 26 \#2520.

113. - On groups with finitely many indecomposable integral representations, Bull. Amer. Math. Soc. 68 (1962), 210-212. MR 25 \#1222.

114. - Grothendieck groups of orders in semisimple algebras, Trans. Amer. Math. Soc. 112 (1964), 344-355. MR 28 \#5093.

115. — Grothendieck groups of integral group rings, Illinois J. Math. 9 (1965), 349-360. MR 31 \#211.

116. D. G. Higman, Indecomposable representations at characteristic p, Duke Math. J. 21 (1954), 377-381. MR 16, 794.

117. — Induced and produced modules, Canad. J. Math. 7 (1955), 490-508. MR 19, 390.

118. - On orders in separable algebras, Canad. J. Math. 7 (1955), 509-515. MR 19, 527.

119. - Relative cohomology, Canad. J. Math. 9 (1957), 19-34. MR 18, 715. 120. - On isomorphisms of orders, Michigan Math. J. 6 (1959), 255-257. MR $22 \# 62$. 
121. - On representations of orders over Dedekind domains, Canad. J. Math. 12 (1960), 107-125. MR 22 \#63.

122. D. G. Higman and J. E. MacLaughlin, Finiteness of class numbers of representations of algebras over function fields, Michigan Math. J. 6 (1959), 401-404. MR 22 \#39.

123. G. Higman, The units of group-rings, Proc. London Math. Soc. (2) 46 (1940), 231-248. MR 2, 5 .

124. R. Holvoet, Sur l'isomorphie d'algèbres de groupes, Bull. Soc. Math. Belg. 20 (1968), 264-282.

124a. D. A. Jackson, On a problem in the theory of integral group rings, Ph.D. thesis, Oxford University, Oxford, 1967.

$124 \mathrm{~b}$. - The group of units of the integral group ring of finite metabelian and finite nilpotent groups, Quart. J. Math. 20 (1969), 319-331.

125. H. Jacobinski, Über die Hauptordnung eines Körpers als Gruppenmodul, J. Reine Angew. Math. 213 (1963/64), 151-164. MR 29 \#1200.

126. - On extensions of lattices, Michigan Math. J. 13 (1966), 471-475. MR 34 \#4377.

127. - Sur les ordres commutatifs avec un nombre fini de réseaux indécomposables, Acta Math. 118 (1967), 1-31. MR 35 \#2876.

128. - Über die Geschlechter von Gittern über Ordnungen, J. Reine Angew. Math. 230 (1968), 29-39. MR 37 \#5250.

129. - Genera and decompositions of lattices over orders, Acta. Math. 121 (1968), 1-29.

130. - On embedding of lattices belonging to the same genus, Proc. Amer. Math. Soc. 24 (1970), 134-136.

131. N. Jacobson, The theory of rings, Math. Surveys, no. II, Amer. Math. Soc., Providence, R. I., 1943. MR 5, 31.

132. - Structure of rings, Amer. Math. Soc. Colloq. Publ., vol. 37, Amer. Math. Soc., Providence, R. I., 1956. MR 18, 373.

133. W. E. Jenner, Block ideals and arithmetics of algebras, Compositio Math. 11 (1953), 187-203. MR 16, 7.

134. - On the class number of non-maximal orders in $९$-adic division algebras, Math. Scand. 4 (1956), 125-128. MR 18, 375.

135. A. Jones, Groups with a finite number of indecomposable integral representations, Michigan Math. J. 10 (1963), 257-261. MR 27 \#3698.

136. - Integral representations of the direct product of groups, Canad. J. Math. 15 (1963), 625-630. MR 27 \#4870.

137. - On representations of finite groups over valuation rings, Illinois J. Math. 9 (1965), 297-303. MR 31 \#257.

138. I. Kaplansky, Elementary divisors and modules, Trans. Amer. Math. Soc. 66 (1949), 464-491. MR 11, 155.

139. - Modules over Dedekind rings and valuation rings, Trans. Amer.

Math. Soc. 72 (1952), 327-340. MR 13, 719.

140. - Submodules of quaternion algebras, Proc. London Math. Soc. (3) 19 (1969), 219-232.

141. V. V. Kiričenko, Orders whose representations are all completely reducible, Mat. Zametki 2 (1967), 139-144 = Math. Notes 2 (1967), 567-570. MR 36 \#2609.

142. D. I. Knee, The indecomposable integral representations of finite cyclic groups, Ph.D. Thesis, M.I.T., Cambridge, Mass., 1962. 
143. M. Kneser, Einige Bemerkungen über ganzzahlige Darstellungen endlicher Gruppen, Arch. Math 17 (1966), 377-379. MR 34 \#1408.

144. S. A. Krugljak, Exact ideals in a second order integral matrix ring, Ukrain. Mat. Ž. 18 (1966), no. 3, 58-64. (Russian) MR 33 \#7378.

145. — The Grothendieck group, Ukrain. Mat. Ž. 18 (1966), no. 5, 100-105. (Russian) MR 34 \#204.

146. T. Y. Lam, Induction theorems for Grothendieck groups and Whitehead groups of finite groups, Ann. Sci. Ecole Norm. Sup. (4) 1 (1968), 91-148.

147. R. Larson, Group rings over Dedekind domains. I, II, J. Algebra 5 (1967), 358-361; 7 (1967), 278-279. MR 35 \#266; MR 35 \#5525.

148. C. G. Latimer and C. C. MacDuffee, $A$ correspondence between classes of ideals and classes of matrices, Ann. of Math, (2) 34 (1933), 313-316.

149. W. J. Leahey, The classification of the indecomposable integral representations of the dihedral group of order 2p, Ph.D. Thesis, M.I.T., Cambridge, Mass., 1962.

150. M. P. Lee, Integral representations of dihedral groups of order $2 p$, Trans. Amer. Math. Soc. 110 (1964), 213-231. MR 28 \#139.

151. H. Leopoldt, Über die Hauptordnung der ganzen Elemente eines abelschen Zahlkörpers, J. Reine Angew. Math. 201 (1959), 119-149. MR 21 \#7195.

152. L. S. Levy, Decomposing pairs of modules, Trans. Amer. Math. Soc. 122 (1966), 64-80. MR 33 \#2677.

153. G. W. Mackey, On induced representations of groups, Amer. J. Math. 73 (1951), 576-592. MR 13, 106.

154. J.-M. Maranda, On $\mathfrak{P}$-adic integral representations of finite groups, Canad. J. Math. 5 (1953), 344-355. MR. 15, 100.

155. - On the equivalence of representations of finite groups by groups of automorphisms of modules over Dedekind rings, Canad. J. Math. 7 (1955), 516-526. MR 19, 529.

155a. J. Martinet, Sur l'arithmétique des extensions galoisiennes d̀ groupe de Galois diédral d'ordre 2p, Ann. Inst. Fourier (Grenoble) 19 (1969) (to appear).

156. A. Matuljauskas, Integral representations of a fourth-order cyclic group, Litovsk. Mat. Sb. 2 (1962), no. 1, 75-82. (Russian) MR 26 \#6274.

157. - Integral representations of the cyclic group of order six, Litovsk. Mat. Sb. 2 (1962), no. 2, 149-157. (Russian) MR 27 \#5835.

158. - On the number of indecomposable representations of the group $Z_{8}$, Litovsk. Mat. Sb 3 (1963), no. 1, 181-188. (Russian) MR 29 \#2309.

159. A. Matuljauskas and M. Matuljauskene, On integral representations of a group of type (3, 3), Litovsk. Mat. Sb. 4 (1964), 229-233. (Russian) MR 29 \#4812.

160. Warren May, Commutative group algebras, Trans. Amer. Math. Soc. 136 (1969), 139-149. MR 38 \#2224.

160a. G. O. Michler, Structure of semi-perfect hereditary noetherian rings, J. Algebra 13 (1969), 327-344.

161. T. Nakayama, $A$ theorem on modules of trivial cohomology over a finite group, Proc. Japan Acad. 32 (1956), 373-376. MR 18, 191.

162. - On modules of trivial cohomology over a finite group, Illinois J. Math. 1 (1957), 36-43. MR 18, 793.

163. - On modules of trivial cohomology over a finite group. II: Finitely generated modules, Nagoya Math. J. 12 (1957), 171-176. MR 20 \#4587.

164. L. A. Nazarova, Unimodular representations of the four group, Dokl. Akad. Nauk SSSR 140 (1961), 1011-1014 =Soviet Math. Dokl. 2 (1961), 1304-1307. MR 24 \#A770. 
165. - Unimodular representations of the alternating group of degree four, Ukrain. Mat. Ž. 15 (1963), 437-444. (Russian) MR 28 \#2148.

166. - Representations of a tetrad, Izv. Akad. Nauk SSSR Ser. Mat. 31 (1967), 1361-1378 = Math. USSR Izv. 1 (1967), 1305-1322. MR 36 \#6400.

167. L. A. Nazarova and A. V. Rorter, Integral representations of the symmetric group of third degree, Ukrain. Mat. Ž. 14 (1962), 271-288. (Russian) MR 26 \#6273.

168. - On irreducible representations of p-groups over $Z_{p}(\epsilon)$, Ukrain. Mat. Ž. 18 (1966), no 1, 119-124. (Russian) MR 34 \#254.

169. - On integral p-adic representations and representations over residue class rings, Ukrain. Mat. Ž. 19 (1967), no. 2, 125-126. (Russian) MR 35 \#267.

170. - Refinement of a theorem of Bass, Dokl. Akad. Nauk SSSR 176 (1967), 266-268=Soviet Math. Dokl. 8 (1967), 1089-1092. MR 37 \#1402.

171. - Finitely generated modules over a dyad of a pair of local Dedekind rings, and finite groups having an abelian normal subgroup of index $p$, Izv. Akad. Nauk SSSR Ser. Mat. 33 (1969), 65-89 = Math. USSR Izv. 3 (1969).

172. M. Newman and O. Taussky, Classes of positive definite unimodular circulants, Canad. J. Math. 9 (1957), 71-73. MR 18, 634.

173. - On a generalization of the normal basis in abelian algebraic number fields, Comm. Pure Appl. Math. 9 (1956), 85-91. MR 17, 829.

174. R. J. Nunke, Modules of extensions over Dedekind rings, Illinois J. Math. 3 (1959), 222-241. MR 21 \#1329.

175. T. Obayashi, On the Grothendieck ring of an abelian p-group, Nagoya Math. J. 26 (1966), 101-113. MR 37 \#1438.

176. J. Oppenheim, Integral representations of cyclic groups of squarefree order, Ph.D. Thesis, University of Illinois, Urbana, Ill., 1962.

177. D. S. Passman, Nil ideals in group rings, Michigan Math. J. 9 (1962), 375384. MR $26 \# 2470$.

178. - Isomorphic groups and group rings, Pacific J. Math. 15 (1965), 561-583. MR 33 \#1381.

179. L. C. Pu, Integral representations of non-abelian groups of order $p q$, Michigan Math. J. 12 (1965), 231-246. MR 31 \#2321.

180. T. Ralley, Decomposition of products of modular representations, J. London Math. Soc. 44 (1969), 480-484.

181. I. Reiner, Maschke modules over Dedekind rings, Canad. J. Math. 8 (1956), 329-334. MR 18, 7.

182. - Integral representations of cyclic groups of prime order, Proc. Amer. Math. Soc. 8 (1957), 142-146, MR 18, 717.

183. - On the class number of representations of an order, Canad. J. Math. 11 (1959), 660-672. MR 21 \#7229.

184. - The nonuniqueness of irreducible constituents of integral group representations, Proc. Amer. Math. Soc. 11 (1960), 655-658. MR 23 \#A223.

185. - Behavior of integral group representations under ground ring extension, Illinois J. Math. 4 (1960), 640-651. MR 22 \#12145.

186. - The Krull-Schmidt theorem for integral group representations, Bull. Amer. Math. Soc. 67 (1961), 365-367. MR 25 \#2132.

187. - Indecomposable representations of non-cyclic groups, Michigan Math. J. 9 (1962), 187-191. MR 25 \#3994.

188. - Failure of the Krull-Schmidt theorem for integral representations, Michigan Math. J. 9 (1962), 225-231. MR 26 \#2482. 
189. - Extensions of irreducible modules, Michigan Math J. 10 (1963), 273276. MR $27 \# 5807$.

190. — The integral representation ring of a finite group, Michigan Math. J. 12 (1965), 11-22. MR $30 \# 3152$.

191. - Nilpotent elements in rings of integral representations, Proc. Amer. Math. Soc. 17 (1966), 270-274. MR 32 \#5745.

192. — Integral representation algebras, Trans. Amer. Math. Soc. 124 (1966), 111-121. MR 34 \#2722.

193. - Relations between integral and modular representations, Michigan Math. J. 13 (1966), 357-372. MR 36 \#5240.

194. - Module extensions and blocks, J. Algebra 5 (1967), 157-163. MR 35 \#4316.

195. - Representation rings, Michigan Math. J. 14 (1967), 385-391. MR $36 \# 1555$.

196. - An involution on $\tilde{K}^{0}(Z G)$, Mat. Zametki 3 (1968), 523-527. (Russian) MR 37 \#5270.

197. - A survey of integral representation theory, Proc. Algebra Sympos., University of Kentucky (Lexington, 1968), pp. 8-14.

198. - Maximal orders, Mimeograph Notes, University of Illinois, Urbana, III., 1969.

199. I. Reiner and H. Zassenhaus, Equivalence of representations under extensions of local ground rings, lllinois J. Math. 5 (1961), 409-411. MR 23 \#A3764.

200. D. S. Rim, Modules over finite groups, Ann. of Math. (2) 69 (1959), 700-712. MR 21 \#3474.

201. - On projective class groups, Trans. Amer. Math. Soc. 98 (1961), 459467. MR 23 \#A1690.

202. K. W. Roggenkamp, Gruppenringe von unendlichem Darstellungstyp, Math. Z. 96 (1967), 393-398. MR 34 \#5948.

203. - Darstellungen endlicher Gruppen in Polynomringen, Math. Z. 96 (1967), 399-407. MR 34 \#5949.

204. - Grothendieck groups of hereditary orders, J. Reine Angew. Math. 235 (1969), 29-40.

205. - On the irreducible lattices of orders, Canad. J. Math. 21 (1969), 970976.

206. - Das Krull-Schmidt Theorem für projektive Gitter in Ordnungen über lokalen Ringen, Math. Seminar (Giessen, 1969).

207. — Projective modules over clean orders, Compositio Math. 21 (1969), 185-194.

208. - A necessary and sufficient condition for orders in direct sums of complete skewfields to have only finitely many nonisomorphic indecomposable integral representations, Bull. Amer. Math. Soc. 76 (1969), 130-134.

208a. - Projective homorphisms and extensions of lattices, J. Reine Angew. Math. (to appear).

208b. K. W. Roggenkamp and V. H. Dyson, Modules over orders, Springer Lecture Notes (to appear).

209. A. V. Royter, On the representations of the cyclic group of fourth order by integral matrices, Vestnik Leningrad. Univ. 15 (1960), no. 19, 65-74. (Russian) MR 23 \#A1730.

210. - Categories with division and integral representations, Dokl. Akad. Nauk SSSR 153 (1963), 46-48=Soviet Math. Dokl. 4 (1963), 1621-1623. MR 33 $\# 2704$. 
211. - On a category of representations, Ukrain. Mat. Ž. 15 (1963), 448-452. (Russian) MR $28 \# 3072$.

212. - On integral representations belonging to a genus, Izv. Akad. Nauk SSSR Ser. Mat. 30 (1966), 1315-1324; English transl., Amer. Math. Soc. Transl. (2) 71 (1968), 49-59. MR 35 \#4255.

213. - Divisibility in the category of representations over a complete local Dedekind ring, Ukrain. Mat. Ž. 17 (1965), no. 4, 124-129. (Russian) MR 33 \#5699.

214. —, E-systems of representations, Ukrain. Mat. Ž. 17 (1965), no. 2, 88-96. (Russian) MR $32 \# 7620$.

215. — An analog of Bass' theorem for representation modules of non-commutative orders, Dokl. Akad. Nauk SSSR 168 (1966), 1261-1264=Soviet Math. Dokl. 7 (1966), 830-833. MR 34 \#2632.

216. - Unboundedness of the dimensions of indecomposable representations of algebras having infinitely many indecomposable representations, Izv. Akad. Nauk SSSR Ser. Mat. 32 (1968), 1275-1282 = Math. USSR Izv. 2 (1968) (to appear).

217. - On the theory of integral representations of rings, Mat. Zametki 3 (1968), 361-366. (Russian) MR 38 \#187.

218. J. Rotman, Homological algebra, Van Nostrand, Princeton, N. J., 1970.

219. V. P. Rud'ko, On the integral representation algebra of a cyclic group of order $p^{2}$,

Dopovidi Akad. Nauk Ukrain. RSR Ser. A 1967, 35-39. (Ukrainian) MR 35 \#268.

220. A. I. Saksonov, On group rings of finite p-groups over certain integral domains,

Dokl. Akad Nauk BSSR 11 (1967), 204-207. (Russian) MR 35 \#270.

221. - Group-algebras of finite groups over a number field, Dokl. Akad. Nauk BSSR 11 (1967), 302-305. MR 35 \#1681.

222. O. F. G. Schilling, The theory of valuations, Math. Surveys, no. 4, Amer. Math. Soc., Providence, R. I., 1950. MR 13, 315.

223. $\mathrm{H}$. Schneider and J. Weissglass, Group rings, semigroup rings and their radicals, J. Algebra 5 (1967), 1-15. MR 35 \#317.

224. S. K. Sehgal, On the isomorphism of integral group rings. I, II, Canad. J. Math. 21 (1969), 410-413, 1182-1188.

225. C. S. Seshadri, Triviality of vector bundles over the affine space $K^{2}$, Proc. Nat. Acad. Sci. U. S. A. 44 (1958), 456-458. MR 21 \#1318.

226. - Algebraic vector bundles over the product of an affine curve and the affine line, Proc. Amer. Math. Soc. 10 (1959), 670-673. MR 29 \#2263.

226a. M. Singer, Invertible powers of ideals over orders in commutative separable algebras, Proc. Cambridge Philos. Soc. (to appear).

227. D. L. Stancl, Multiplication in Grothendieck rings of integral group rings, J. Algebra 7 (1967), 77-90. MR 36 \#6476.

228. E. Steinitz, Rechteckige Systeme und Moduln in algebraischen Zahlenkörpern.

I, Il, Math. Ann. 71 (1911), 328-354; 72 (1912), 297-345.

229. J. R. Strooker, Faithfully projective modules and clean algebras, $\mathrm{Ph} . \mathrm{D}$. Thesis, University of Utrecht, 1965.

230. R. G. Swan, Projective modules over finite groups, Bull. Amer. Math. Soc. 65 (1959), 365-367. MR 22 \#5660.

231. - The p-period of a finite group, Illinois J. Math. 4 (1960), 341-346. MR 23 \#A188.

232. - Induced representations and projective modules, Ann. of Math. (2) 71 (1960), 552-578. MR 252131.

233. - Projective modules over group rings and maximal orders, Ann. of Math. (2) 76 (1962), 55-61. MR 25 \#3066. 
234. MR 27 \#3683.

235. - Algebraic K-theory, Springer Lecture Notes, Berlin, 1968.

236. - Invariant rational funclions and a problem of Steenrod, Invent. Math. 7 (1969), 148-158.

237. ㄴ, The number of generators of a module, Math. Z. 102 (1967), 318-322. MR 36 \#1434.

238. S. Takahashi, Arithmetic of group representations, Tôhoku Math. J. (2) 11 (1959), 216-246. MR 22 \#733.

239. - A characterization of group rings as a special class of Hopf algebras, Canad. Math. Bull. 8 (1965), 465-475. MR 32 \#2459.

240. O. Taussky, On a theorem of Latimer and MacDuffee, Canad. J. Math. 1 (1949), 300-302. MR 11, 3.

241. Classes of matrices and quadratic fields, Pacific J. Math. 1 (1951), 127-132. MR 13, 201.

242. - Classes of matrices and quadratic fields. II, J. London Math. Soc. 27 (1952), 237-239. MR 13, 717.

243. - Unimodular integral circulants, Math. Z. 63 (1955), 286-289. MR $17,347$.

244. - On matrix classes corresponding to an ideal and its inverse, Illinois J. Math. 1 (1957), 108-113. MR $20 \# 845$.

245. - Matrices of rational integers, Bull. Amer. Math. Soc. 66 (1960), 327-345. MR 22 \#10994.

246. - Ideal matrices. I, Arch. Math. 13 (1962), 275-282. MR 27 \#168.

247. - Ideal matrices. II, Math. Ann. 150 (1963), 218-225. MR 28 \#105.

248. - On the similarity transformation between an integral matrix with irreducible characteristic polynomial and its transpose, Math. Ann. 166 (1966), 60-63. MR 33 \#7355.

249. - The discriminant matrices of an algebraic number field, J. London Math. Soc. 43 (1968), 152-154. MR 37 \#4053.

250. O. Taussky and J. Todd, Matrices with finite period, Proc. Edinburgh Math. Soc. (2) 6 (1940), 128-134. MR 2, 118.

251. - Matrices of finite period, Proc. Roy. Irish Acad. Sect. A 46 (1941), 113-121. MR 2, 243.

252. O. Taussky and $\mathrm{H}$. Zassenhaus, On the similarity transformation between a matrix and its transpose, Pacific J. Math. 9 (1959), 893-896. MR 21 \#7216.

253. John G. Thompson, Vertices and sources, J. Algebra 6 (1967), 1-6. MR 34 \#7677.

254. A. Troy, Integral representations of cyclic groups of order $p^{2}, \mathrm{Ph} . \mathrm{D}$. Thesis, University of Illinois, Urbana, Ill., 1961.

255. K. Uchida, Remarks on Grothendieck groups, Tôhoku Math. J. (2) 19 (1967), 341-348. MR 37 \#2838.

256. S. Ullom, Normal bases in Galois extensions of number fields, Nagoya Math. J. 34 (1969), 153-167.

257. - Galois cohomology of ambiguous ideals, J. Number Theory 1 (1969), 11-15.

258. Y. Watanabe, The Dedekind different and the homological different, Osaka J. Math. 4 (1967), 227-231. MR 37 \#2795.

259. - The Dedekind different and the homological different of an algebra, J. Math. Soc. Japan (to appear). 
260. A. Weil, Basic number theory, Die Grundlehren der math. Wissenschaften, Band 114, Springer-Verlag, New York, 1967. MR 38 \#3244.

261. A. R. Whitcomb, The group ring problem, $\mathrm{Ph} . \mathrm{D}$. thesis, University of Chicago, Chicago, Ill., 1968.

262. O. Zariski and P. Samuel, Commutative algebra. Vol. I, University Series in Higher Math., Van Nostrand, Princeton, N. J., 1958. MR 19, 833.

263. H. Zassenhaus, Neuer Beweis der Endlichkeit der Klassenzahl bei unimodularer Aquivalenz endlicher ganzzahliger Substitutionsgruppen, Abh. Math. Sem. Univ. Hamburg 12 (1938), 276-288.

264. - Über die Äquivalenz ganzzahliger Darstellungen, Nachr. Akad. Wiss. Göttingen Math.-Phys. Kl. II 1967, 167-193. MR 37 \#6319.

265. J. Zemanek, On the semisimplicity of integral representation rings, Ph.D. Thesis, University of Illinois, Urbana, Ill., 1970.

UnIVERSITy of ILlinoIs, URbana, ILlinoIs 61801 